\title{
THERMOREGULATION OF THE TESTICLE IN RESPONSE TO EXERCISE AND SUBSEQUENT EFFECTS ON SEMINAL CHARACTERISTICS IN STALLIONS
}

\author{
A Thesis \\ by \\ JEANNETTE DIANE MAWYER \\ Submitted to the Office of Graduate Studies of \\ Texas A\&M University \\ in partial fulfillment of the requirements for the degree of \\ MASTER OF SCIENCE
}

May 2011

Major Subject: Animal Science 
Thermoregulation of the Testicle in Response to Exercise and Subsequent Effects on Seminal Characteristics in Stallions Copyright 2011 Jeannette Diane Mawyer 


\title{
THERMOREGULATION OF THE TESTICLE IN RESPONSE TO EXERCISE AND SUBSEQUENT EFFECTS ON SEMINAL CHARACTERISTICS IN STALLIONS
}

\author{
A Thesis \\ by \\ JEANNETTE DIANE MAWYER
}

\begin{abstract}
Submitted to the Office of Graduate Studies of Texas A\&M University

in partial fulfillment of the requirements for the degree of

MASTER OF SCIENCE
\end{abstract}

Approved by:

Chair of Committee, Clay A. Cavinder

Committee Members, Martha M. Vogelsang

Charles C. Love

Steven P. Brinsko

Head of Department, H. Russell Cross

May 2011

Major Subject: Animal Science 


\begin{abstract}
Thermoregulation of the Testicle in Response to Exercise and Subsequent Effects on Seminal Characteristics in Stallions. (May 2011) Jeannette Diane Mawyer, B.S.A., Murray State University Chair of Advisory Committee: Dr. Clay A. Cavinder
\end{abstract}

Studies performed on stallions have characterized detrimental effects on semen quality resulting from thermal stress by testicular insulation, but few have investigated the effects of exercise-induced increases in core body temperature on stallion semen parameters. To our knowledge, this is the first study that correlates subcutaneous scrotal temperature and stallion spermatozoa quality using a subdermal scrotal thermal sensory device. Stallions were assigned to a non-exercised (non-ex; control; $n=4$ ) or exercised (ex; $\mathrm{n}=4$ ) group. A motorized equine exerciser was used to work stallions $30 \mathrm{~min} / \mathrm{d}$ for 4 d/wk during a 12-wk period from July through October. Temperatures (subcutaneous scrotal, subcutaneous neck, rectal, and ambient) were recorded before exercise, immediately after exercise, and 60 and 120 min post-exercise. Humidity data were obtained later to determine THI.

No deleterious effects were observed from implantation of thermal sensory devices. An interaction of treatment and time $(\mathrm{P}<0.0001)$ was evident for rectal and neck temperatures. The relationship between scrotal and rectal temperatures was highest $\left(r_{\mathrm{s}}=0.761\right)$, and other correlations existed between scrotal, neck, and ambient 
temperatures, as well. Mean rectal temperature in the ex group increased $1.9^{\circ} \mathrm{C}(\mathrm{P}<$ $0.0001)$, while there was a slight increase in scrotal temperature of $0.8^{\circ} \mathrm{C}(\mathrm{P}>0.05)$ from $0 \mathrm{~min}$ to $22 \mathrm{~min}$. Although an increase in core body temperature was successfully induced by exercise protocol, scrotal temperatures were not significantly affected, and no treatment effects were found in any of the semen parameters measured $(\mathrm{P}>0.05)$. Therefore, no significant changes in fresh or cooled semen parameters resulted from exercise or increases in core body temperature resulting from exercise protocol used in this study.

Results of this study indicate that thermal sensory devices are a safe and effective way to measure subcutaneous scrotal and neck temperatures. Although an increase in core body temperature was successfully induced by exercise protocol, scrotal temperatures were not significantly affected, indicating efficient thermoregulation of the testes by the scrotum. Since the testes experienced no significant thermal insult during the exercise protocol, no significant changes in fresh or cooled semen parameters were evident as a result of exercise or elevated core temperature. 


\section{DEDICATION}

This thesis is dedicated to the two people I admire most, my parents. They forged the career path that I have chosen in life by providing me the opportunity to grow up with horses. Although this path has taken me far from home and family, I know that no matter the distances I travel, the unwavering love and support of my parents will always sustain me. Mom and Dad, I will forever be grateful for the sacrifices you have made to see me achieve my goals. Thank you; I love you; and I miss you every day.

To my beloved horse and friend of only 5 short years: you will always be the horse to which I will compare others. Your beauty, talent, and personality won the respect of everyone we encountered during and after our years in Murray, and I am quite certain that I will never find a more perfect horse. I miss you, Boomer.

"When we think of those companions who traveled by our side down life's road, let us not say with sadness that they left us behind, but rather say with gentle gratitude that they once were with us." - Author Unknown 


\section{ACKNOWLEDGEMENTS}

First and foremost, I would like to extend my sincere gratitude to my committee chair, Dr. Cavinder, for taking a chance on me and having faith in my ability to succeed as a graduate student and a teaching assistant. These opportunities have enabled me to learn more than I ever could have imagined, and I am forever grateful.

Thanks go to my committee members: Dr. Vogelsang for her willingness to help in any situation at any time; Dr. Love for his patience and willingness to assist with laboratory and statistical analysis; and Dr. Brinsko for his quick advice whenever I was in a bind and for his ability to explain complicated statistical concepts in an understandable manner. Additionally, I would like to thank Dr. Sigler for assisting with planning the exercise physiology-related portions of the current study and Russell Gordon for assisting with all aspects of the project.

Everyone working in the Theriogenology Laboratory deserves many thanks, especially Sheila Teague for being extremely helpful with semen analysis. I thoroughly enjoyed my time there. Thanks also go to my undergraduate student workers; I could not have completed this project without them. Another group that was absolutely essential to the completion of this study consisted of Krissy Schroeder, Carrie Mueller, Stephanie Standridge, Lindsey Huth, Kelly Winsco, and Jennifer Rosenberg. Many thanks go to them for not only assisting with semen collection and analysis, but also for being great friends. Finally, thanks to my parents for their continued love, support, and encouragement. 


\section{NOMENCLATURE}

AA

$\mathrm{AH}$

AI

AM

AMBT

ART

AV

BCS

$\mathrm{BM}$

bpm

BT

BW

CONC

DD

DH

DSO

$\mathrm{EF}$

EP

ex

HR
Abnormal acrosome; spermatozoal morphological abnormality

Abnormal head; spermatozoal morphological abnormality

Artificial insemination

Abnormal midpiece; spermatozoal morphological abnormality

Ambient temperature

Artificial reproductive technologies

Artificial vagina

Body condition score

Bent midpiece; spermatozoal morphological abnormality

Heartbeats per minute

Bent tail; spermatozoal morphological abnormality

Body weight

Concentration of spermatozoa

Distal droplet; spermatozoal morphological abnormality

Detached head; spermatozoal morphological abnormality

Daily spermatozoa output

Extractable fat

Exercise period

Exercised (treatment) group of stallions

Heart rate 


\begin{tabular}{|c|c|}
\hline HUM & Relative humidity \\
\hline ITT & Intratesticular temperature \\
\hline non-ex & Non-exercised (control) group of stallions \\
\hline NORMCELL & Morphologically normal spermatozoa \\
\hline $\mathrm{PD}$ & Proximal droplet; spermatozoal morphological abnormality \\
\hline PGC & Premature germ cell; spermatozoal morphological abnormality \\
\hline PMOT & Progressive motility of spermatozoa \\
\hline post-EP & Post-exercise period; after the cessation of the exercise period \\
\hline pre-EP & Pre-exercise period; prior to the onset of the exercise period \\
\hline $\mathrm{RCT}$ & Rectal temperature \\
\hline rf & Rump fat \\
\hline $\mathrm{RR}$ & Respiration rate \\
\hline SCSA & Sperm Chromatin Structure Assay \\
\hline COMPat & Sperm chromatin structure assay - cells outside main population \\
\hline SET & Standard exercise test \\
\hline SQNT & Subcutaneous neck temperature \\
\hline SQST & Subcutaneous scrotal temperature \\
\hline SST & Scrotal surface temperature \\
\hline T0 & Time 0 ; fresh semen sample \\
\hline $\mathrm{T} 24$ & Time 24; 24-h cooled semen \\
\hline $\mathrm{T} 48$ & Time 48; 48-h cooled semen \\
\hline $\mathrm{TL}$ & Testicular degeneration \\
\hline
\end{tabular}


THI

TMOT

TV

VAP

VIAB

VOL
Temperature-heat index

Total motility of spermatozoa

Testicular volume

Average path velocity of spermatozoa

Viability of spermatozoa

Semen volume 


\section{TABLE OF CONTENTS}

Page

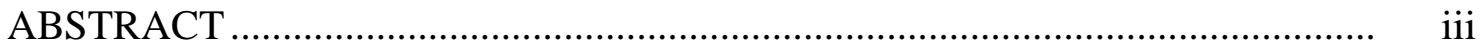

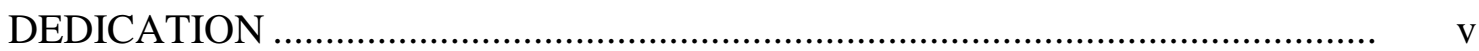

ACKNOWLEDGEMENTS ................................................................. vi

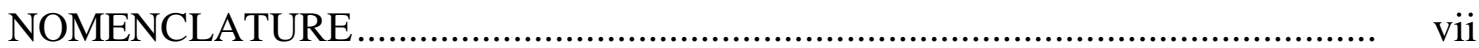

TABLE OF CONTENTS .........................................................................

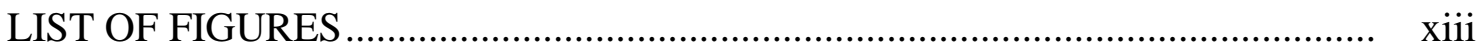

LIST OF TABLES ..........................................................................

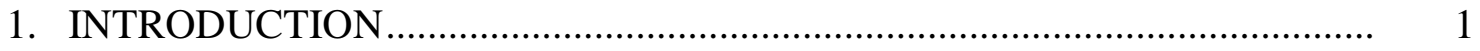

2. LITERATURE REVIEW ................................................................... 5

2.1 Common Causes of Testicular Degeneration.................................... 5

2.2 Relationship between Scrotal Surface and Subcutaneous Scrotal Temperatures and Intratesticular Temperatures ............................... 5

2.3 Influence of Increased Subcutaneous Scrotal Temperature on Reproductive Parameters............................................................... 6

2.4 Influence of Increased Scrotal Surface Temperature on Reproductive Parameters ................................................................................... 7

2.5 Influence of Increased Ambient Temperature on Reproductive Parameters ...................................................................................... 8

2.6 Influence of Exercise on Reproductive Parameters of Other Species.. 10

2.7 Influence of Exercise on Stallion Reproductive Parameters ................ 11

2.8 Exercise and Related Increases in Core Body Temperatures in the

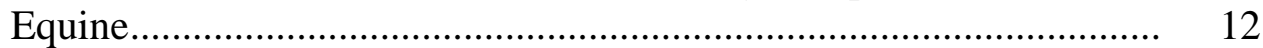

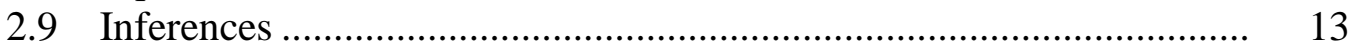




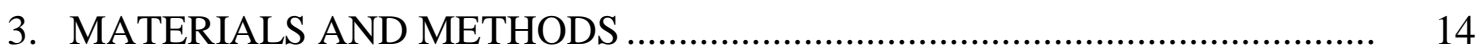

3.1 Stallions, Housing, and Diets ............................................................... 14

3.2 Initial Rest/Acclimation Period ....................................................... 15

3.3 Thermal Sensor Implants ............................................................... 15

3.4 Predicted Daily Spermatozoa Output ................................................. 16

3.5 Blocking and Treatment Groups ......................................................... 17

3.6 Fitness Evaluation - Standard Exercise Tests ................................... 18

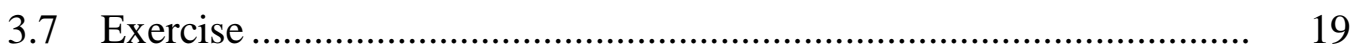

$3.8 \quad$ Semen Collection ..................................................................... $\quad 20$

3.9 Semen Processing ......................................................................... 21

3.10 Semen Analysis ............................................................................ 22

3.11 Statistical Analysis ........................................................................ 25

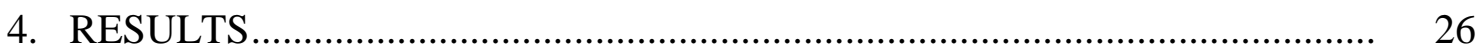

4.1 Implantation of Subcutaneous Scrotal Devices.................................... 26

4.2 Ambient Temperature, Humidity, and Temperature-Heat Index ......... 26

4.3 Changes in Stallion Temperatures Due to Exercise .............................. 27

4.4 Correlation Analysis between Different Temperature Recordings ...... 31

4.5 Fresh Semen ............................................................................... 31

4.6 Cooled Semen ....................................................................... 33

4.7 Evaluation of Fitness Level................................................................ $\quad 35$

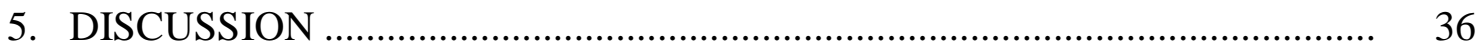

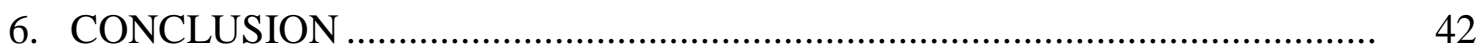

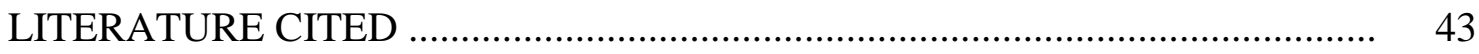

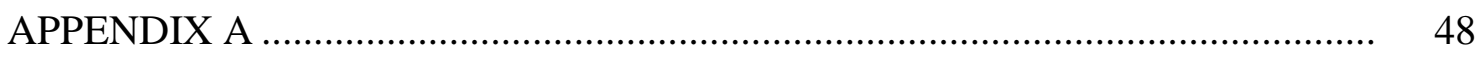

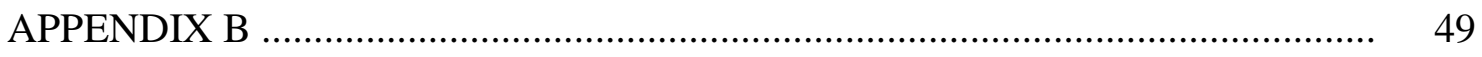

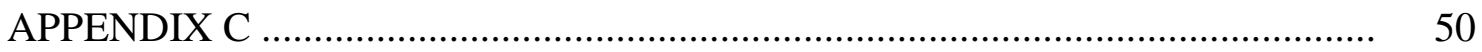

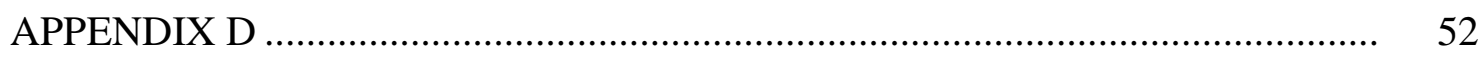

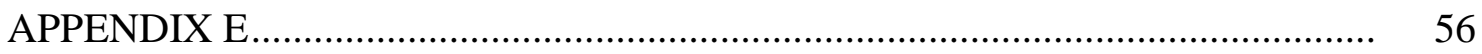




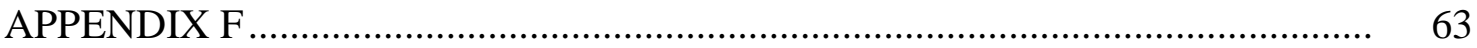

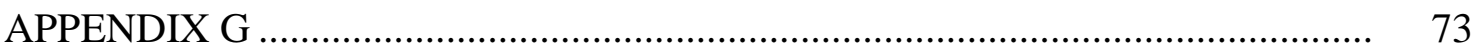

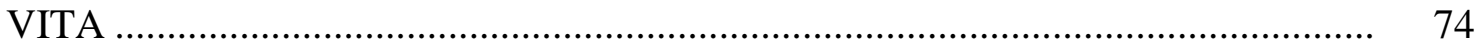




\section{LIST OF FIGURES}

FIGURE Page

1 Mean ambient (AMBT) and temperature-heat index (THI) to which all stallions were subjected at each time point during exercise protocol .......

2 Mean rectal and neck temperatures for non-ex (control) and ex (treatment) stallions during the exercise period ( $\mathrm{n}=4$ /group)

3 Mean rectal and scrotal temperatures for non-ex (control) and ex (treatment) stallions during the exercise period ( $\mathrm{n}=4$ /group)

C.1 Variations in mean temperature (neck, scrotal, and rectal) of individual stallions within the non-exercised (control) group $(n=4)$.

C.2 Variations in mean temperature (neck, scrotal, and rectal) of individual stallions within the exercised (treatment) group $(n=4)$

D.1 Mean ( \pm SD) gel-free semen volume among non-ex (control) and ex (treatment) groups ( $\mathrm{n}=4$ /group).

D.2 Mean $( \pm$ SD) spermatozoal concentration among non-ex (control) and ex (treatment) groups ( $\mathrm{n}=4$ /group).

D.3 Mean $( \pm \mathrm{SD})$ total number of cells among non-ex (control) and ex (treatment) groups ( $\mathrm{n}=4$ /group).

D.4 Mean ( \pm SD) viability of spermatozoa among non-ex (control) and ex (treatment) groups ( $\mathrm{n}=4$ /group).

D.5 Mean ( \pm SD) total motility of spermatozoa among non-ex (control) and ex (treatment) groups ( $\mathrm{n}=4 /$ group $)$.

D.6 Mean $( \pm \mathrm{SD})$ progressive motility of spermatozoa among non-ex (control) and ex (treatment) groups ( $\mathrm{n}=4 /$ group).

D.7 Mean $( \pm$ SD) COMPat of Sperm Chromatin Structure Assay among non-ex (control) and ex (treatment) groups ( $n=4 /$ group).

D.8 Mean $( \pm \mathrm{SD})$ percentages of normal spermatozoa among non-ex (control) and ex (treatment) groups ( $\mathrm{n}=4 /$ group$)$. 
E.1 Variations in mean gel-free volume $(\mathrm{mL})$ of individual stallions within treatment groups: (a) non-exercised (control; $n=4$ ) and (b) exercised (treatment; $n=4)$.

E.2 Variations in mean spermatozoal concentration (million spermatozoa $/ \mathrm{mL}$ ) of individual stallions within treatment groups:

(a) non-exercised (control; $n=4$ ) and (b) exercised (treatment; $n=4)$.

E.3 Variations in mean total numbers (million spermatozoa) of individual stallions within treatment groups: (a) non-exercised (control; $n=4$ ) and (b) exercised (treatment; $n=4$ ).

E.4 Variations in mean viability (\%) of individual stallions within treatment groups: (a) non-exercised (control; $n=4$ ) and (b) exercised (treatment; $n=4)$.

E.5 Variations in mean total motility (\%) of individual stallions within treatment groups: (a) non-exercised (control; $n=4$ ) and (b) exercised (treatment; $n=4$ ).

E.6 Variations in mean progressive motility (\%) of individual stallions within treatment groups: (a) non-exercised (control; $n=4$ ) and (b) exercised (treatment; $n=4$ ).

E.7 Variations in mean normal spermatozoa (\%) of individual stallions within treatment groups: (a) non-exercised (control; $n=4$ ) and (b) exercised (treatment; $n=4$ ).

F.1 Box plots representing mean percentages of abnormal heads among non-ex (control) and ex (treatment) groups at weeks $0,4,8$, and 12 (n=4/group).....

F.2 Box plots representing mean percentages of abnormal acrosomes among non-ex (control) and ex (treatment) groups at weeks $0,4,8$, and 12 (n=4/group)....

F.3 Box plots representing mean percentages of detached heads among non-ex (control) and ex (treatment) groups at weeks $0,4,8$, and 12 $(n=4 /$ group $)$. 
F.4 Box plots representing mean percentages of proximal droplets among non-ex (control) and ex (treatment) groups at weeks $0,4,8$, and 12 $(n=4 / g r o u p)$.

F.5 Box plots representing mean percentages of distal droplets among non-ex (control) and ex (treatment) groups at weeks $0,4,8$, and 12 $(n=4 /$ group $)$.

F.6 Box plots representing mean percentages of abnormal midpieces among non-ex (control) and ex (treatment) groups at weeks $0,4,8$, and 12 $(n=4 /$ group $)$.

F.7 Box plots representing mean percentages of bent midpieces among non-ex (control) and ex (treatment) groups at weeks $0,4,8$, and 12 (n=4/group)....

F.8 Box plots representing mean percentages of bent tails among non-ex (control) and ex (treatment) groups ( $\mathrm{n}=4$ /group)

F.9 Box plots representing mean percentages of coiled tails among non-ex (control) and ex (treatment) groups at weeks $0,4,8$, and 12 $(n=4 / g r o u p)$.

F.10 Box plots representing mean percentages of premature germ cells among non-ex (control) and ex (treatment) groups at weeks $0,4,8$, and 12 $(n=4 /$ group $)$.

G.1 Mean heart rates (beats per minute) of non-ex (control; $n=4)$ and ex (treatment; $n=4)$ stallions during standard exercise tests prior to (pre-EP) and following (post-EP) the exercise period.

G.2 Mean respiration rates (breaths per minute) of non-ex (control; $n=4$ ) and ex (treatment; $n=4)$ stallions during standard exercise tests prior to (pre-EP) and following (post-EP) the exercise period 


\section{LIST OF TABLES}

TABLE

Page

1 Mean ( \pm SD) body condition endpoints of non-exercised (non-ex; control) and exercised (ex; treatment) stallions at the onset (Week 0) and at the end (Week 12) of the study ( $\mathrm{n}=4$ /group).

2 Predicted daily spermatozoa output (DSO; in billions) for all stallions.....

3 Mean $( \pm \mathrm{SD})$ values of blocking criteria used to assign stallions to non-ex (control) or ex (treatment) groups ( $\mathrm{n}=4 /$ group)

$4 \quad$ Standard exercise test (SET) protocol for all stallions; used before (pre-EP) and after (post-EP) the exercise period (EP).

$5 \quad$ Exercise protocol ( $4 \mathrm{~d} / \mathrm{wk})$ used for ex (treatment) stallions during a 12-wk exercise period (EP), with temperatures recorded at each time point.

6 Mean ambient (AMBT), humidity (HUM), and temperature-heat index (THI) at each time point during exercise.

7 Mean $( \pm \mathrm{SD})$ rectal temperatures $(\mathrm{RCT})$ of non-ex (control) and ex (treatment) stallions during exercise protocol ( $\mathrm{n}=4 /$ group).

8 Mean $( \pm \mathrm{SD})$ subcutaneous neck temperatures (SQNT) of non-ex (control) and ex (treatment) stallions during exercise protocol ( $n=4 /$ group)

9 Mean $( \pm \mathrm{SD})$ subcutaneous scrotal temperatures (SQST) of non-ex (control) and ex (treatment) stallions during exercise protocol (n=4/group).

10 Mean correlations $\left(\mathrm{r}_{\mathrm{s}}\right)$ of subcutaneous scrotal temperature (SQST), subcutaneous neck temperature (SQNT), rectal temperature (RCT), ambient temperature (AMBT), relative humidity (HUM), and temperatureheat index (THI).

11 Mean ( \pm SD) semen characteristics for all samples (T0, T24, and T48) of non-ex (control) and ex (treatment) stallions ( $\mathrm{n}=4$ /group) 
TABLE

Page

12 Mean $( \pm \mathrm{SD})$ percentages of normal spermatozoa and morphologically abnormal spermatozoa observed in non-ex (control) and ex (treatment) stallions at 4 daily spermatozoa output determinations (Week 0, 4, 8, and 12) ( $\mathrm{n}=4$ /group).

13 Mean $( \pm \mathrm{SD})$ of heart rate $(\mathrm{HR})$ and respiration rate $(\mathrm{RR})$ of non-ex (control) and ex (treatment) stallions, recorded at $28 \mathrm{~min}$ of standard exercise tests conducted before exercise period (pre-EP) and upon completion of the exercise period (post-EP) ( $n=4$ /group)

A.1 Timeline of study protocol.

B.1 Chemical composition of forage diet

B.2 Chemical composition of concentrate diet 


\section{INTRODUCTION}

Results of a population estimate conducted during the early 1990s indicated that $25 \%$ of horses in Texas were being used for reproductive purposes (Jones et al., 1993). A 2004 survey determined that Texas is home to more horses than any other state, accounting for approximately $10 \%$ of the U.S. horse population (Deloitte, 2005). Although the more recent study does not report the number of horses used for breeding purposes specifically in Texas, it does acknowledge the importance of breeding on the U.S. equine industry. It specifically reviews the impact of breeding on the racing industry: of the 9.2 billion horses in the U.S., 844,500 are used for racing, and more than half of the horses used for racing are involved in breeding (Deloitte, 2005).

Unlike food animals that are valued for their reproductive abilities, a stallion's most attractive assets are his performance records and his ability to produce offspring with similar performance capabilities. While many stallions are retired to breeding-only activity, others are required to maintain a performance career as well, requiring stallion managers to simultaneously maintain these stallions at a high level of fitness and training while maintaining quality semen. A present concern regards stallions subjected to high levels of activity that may not permit preservation of testicular health and production of maximal sperm quality. A reduction in sperm quality would reduce fertility, especially in processed semen, which would reduce the efficiency of widely-used artificial reproductive technologies (ART). For example, artificial insemination (AI) has proven

This thesis follows the style of the Journal of Animal Science. 
beneficial to the success of the U.S. equine breeding industry; however, it has placed even more of a strain on stallion managers. High quality ejaculates are crucial to maximize the efficiency of cooled/shipped semen to facilitate the use of AI, as the process of extending, cooling or freezing, and shipping semen can be quite taxing on spermatozoa. A stallion with low quality semen may not be a candidate for shipment if his spermatozoa are unlikely to survive. Therefore, stallion managers must carefully control the many factors known to detrimentally affect stallion semen quality in order to optimize reproductive efficiency.

According to McKinnon and Voss (1993), thermal damage is often due to an increase in normal core body temperature of a stallion resulting from various factors such as systemic infection, prolonged increase in ambient temperature, or scrotal insulation from edema, dermatitis, or hemorrhage. Thermal damage is the most common cause of testicular degeneration that can result in subsequent decreased semen characteristics among stallions (McKinnon and Voss, 1993). Although equine breeders are aware that factors such as heat and exercise stress may hinder spermatozoal production, many are forced to subject their stallions to potential damages from these sources during the breeding season in an effort to maintain a level of training required for maximum athletic performance.

Hot and humid environmental temperatures add an additional challenge to stallion managers in south-central Texas, as ambient temperatures can become extremely high $\left(24^{\circ} \mathrm{C}\right.$ to $\left.36^{\circ} \mathrm{C}\right)$ during peak breeding/show season (NOAA, 2010). These temperatures, coupled with high relative humidity, can be taxing on the efficiency of 
sweating and other mechanisms responsible for cooling the body. Maintenance of a lower testicular temperature relative to core body temperature is achieved by means of the thermoregulatory tissues of the testes; namely, the pampiniform plexus, tunica dartos, cremaster muscle, and scrotum. Efficient thermoregulation is necessary for normal spermatogenesis; however, it is unclear whether a threshold exists due to high heat indices coupled with exercise and minimal airflow above which the scrotum and other thermoregulatory tissues are unable to thermoregulate. Many stallions endure rigorous training during the breeding season, which may cause a prolonged increase in body temperature resulting in thermal damage to the testes, leading to a decrease the quality of spermatozoa and potentially lower fertility.

Increased core body temperature achieved during excessive exercise (Webb et al., 1987; Scot, 1992; Kohn et al., 1999) may be a factor contributing to male infertility. Thermometers affixed to the skin of the scrotum have been used in past research to measure the scrotal surface temperatures (SST) of stallions during exercise (Staempfli et al., 2006) or testicular insulation (Love and Kenney, 1999; Blanchard et al., 2000); however, to our knowledge, no literature regarding stallions has documented subcutaneous scrotal temperatures resulting from increases in core body temperature due to exercise. Therefore, the objectives of the current study were to:

1. Determine whether subdermal thermal sensors could be applied to the measurement of rectal, subcutaneous neck, and subcutaneous scrotal temperatures, 
2. Determine the effect of exercise on rectal, subcutaneous neck, and subcutaneous scrotal temperatures of stallions in hot and humid environments,

3. Determine the relationship between changes in rectal, subcutaneous neck, and subcutaneous scrotal temperatures and sperm quality. 


\section{LITERATURE REVIEW}

\subsection{Common Causes of Testicular Degeneration}

Testicular degeneration (TD) can be defined as the process which causes deterioration in the structure of the testis with a consequent loss of testicular function (Oristalgo Turner, 2007). In stallions, TD is a common cause of acquired subfertility and infertility, which results in substantial economic losses due to loss of breeding fees, increased management costs, and loss of valuable male genetics (McKinnon and Voss, 1993). Stallions may experience TD subsequent to certain nutritional deficiencies, administration of exogenous androgens, spermatozoal outflow obstructions, neoplasia, infection, autoimmune disease, testicular trauma, exposure of the testes to heat or cold, radiation, toxins, or ischemia (Oristalgo Turner, 2007). However, the focus of literary review for the current study examined TD and subsequent effects on reproductive parameters in a variety of species relative to high ambient temperatures (AMBT), rectal temperatures (RCT), subcutaneous scrotal temperatures (SQST), SST, or exercise.

\subsection{Relationship between Scrotal Surface and Subcutaneous Scrotal Temperatures}

\section{and Intratesticular Temperatures}

Studies in bulls and rams have used infrared thermography to obtain SST and needle thermistors to obtain SQST and intratesticular temperature (ITT) in order to determine whether correlations existed among the temperatures recorded from different areas of the testes (bulls: Kastelic et al., 1995; rams: Coulter et al., 1988). Relationships 
between SST and SQST were moderate to high (mean $r=0.73$ ), with a mean difference of $3.4^{\circ} \mathrm{C}$; those between SQST and ITT were low to moderate (mean $r=0.45$ ), with a mean difference of $1.3^{\circ} \mathrm{C}$; and those between SST and ITT were low (mean $r=0.29$ ), with a mean difference of $4.7^{\circ} \mathrm{C}$ (Kastelic et al., 1995). These correlations suggest that inferences should not be made about ITT based on other scrotal temperature measurements (Kastelic et al., 1995).

\subsection{Influence of Increased Subcutaneous Scrotal Temperature on Reproductive}

\section{Parameters}

Needle thermistors have been used to measure SQST in rams as opposed to the aforementioned studies that used SST (Mieusset et al., 1992). Rams experienced a 1.4 to $2.2^{\circ} \mathrm{C}$ increase in SQST via scrotal insulation for $21 \mathrm{~d}$ which resulted in a significant increase in embryonic mortality rate (Mieusset et al., 1992). Similar to studies in bulls (Ross and Entwistle, 1979; Vogler et al., 1993) that used SST as the endpoint to measure thermal insult, deleterious effects were apparent as early as d 4 of insulation, suggesting that spermatozoa stored in the epididymis was initially affected, with significant decreases in viability, motility, and total number of spermatozoa subsequently observed (Mieusset et al., 1992).

A study conducted on men working in a steel-casting plant and being exposed to $38.9^{\circ} \mathrm{C}$ for $5 \mathrm{~h} / \mathrm{d}$ found no effects on semen parameters, as internal scrotal temperatures obtained through scrotal invagination thermography of these men did not differ from those of control subjects not exposed to high temperatures. Authors attributed this 
phenomenon to acclimatization of treated subjects (Momen et al., 2010). However, a similar study reported that welders working in temperatures of 31 to $45^{\circ} \mathrm{C}$ for $5 \mathrm{~h} / \mathrm{d}$ for 6 wk increased groin temperature by $1.4^{\circ} \mathrm{C}$, and a subsequent decrease in percent normal spermatozoa was noted (Bonde, 1992).

\subsection{Influence of Increased Scrotal Surface Temperature on Reproductive Parameters}

Scrotal heating or insulation has been used to increase stallion SST by approximately $3^{\circ} \mathrm{C}$ for 24 to $48 \mathrm{~h}$ (Freidman et al., 1991), 3 to $3.5^{\circ} \mathrm{C}$ for $36 \mathrm{~h}$ (Blanchard et al., 2000), and 2 to $3^{\circ} \mathrm{C}$ for $48 \mathrm{~h}$ (Love and Kenney, 1999). Results of these studies showed a decrease in spermatozoal motility, concentration, number of spermatozoa per ejaculate, percentage of morphologically normal spermatozoa (Freidman et al., 1991; Blanchard et al., 2000), as well as decreased DNA quality (Love and Kenney, 1999). Similar studies in bulls and boars maintained SST at $35^{\circ} \mathrm{C}$ for 10 or $20 \mathrm{~h}$ (bulls; Ross and Entwistle, 1979), 33.3 to $36.4^{\circ} \mathrm{C}$ for $48 \mathrm{~h}$ (bulls; Vogler et al., 1993), and $39.5^{\circ} \mathrm{C}$ for $3 \mathrm{~h}$ (boars; McNitt and First, 1970). Results of these studies demonstrated a decrease in spermatozoal concentration and percent live and motile spermatozoa (Ross and Entwistle, 1979; McNitt and First, 1970), as well as an increase in percent abnormal spermatozoa (Ross and Entwistle, 1979; Vogler et al., 1993). Studies aimed at more closely examining the effects of elevated temperature on morphological characteristics in the bovine agree that a 1 to $1.5^{\circ} \mathrm{C}$ increase in SST for 2 or $4 \mathrm{~d}$ interfered with spermatogenic processes, as indicated by observed increases in primary morphologic abnormalities of spermatozoa (Vogler et al., 1993; Barth and Bowman, 1994). After $8 \mathrm{~d}$ 
of insulation, a direct effect of heat on epididymal spermatozoa was apparent, shown by increases in dead spermatozoa and detached heads, which were found to be directly related to loss of cell membrane integrity (Barth and Bowman, 1994). Severity of the changes in seminal characteristics varied in these studies, and no assumptions can be made about the extent of these changes based on duration of thermal stress.

Similar studies conducted on men are consistent with the findings in domestic livestock species. Research investigating male contraception via genital heat stress introduced an apparatus for the immersion of the scrotum into a $45^{\circ} \mathrm{C}$ water bath (Watanabe, 1959). Hot water bath treatment for $30 \mathrm{~min} / \mathrm{d}$ for $12 \mathrm{~d}$ dramatically compromised semen quality for 5 to $12 \mathrm{wk}$, after which time spermatozoal parameters returned to normal (Watanabe, 1959). Men who wore close-fitting underwear that held the testes close to the inguinal canal for 2 to 6 mo experienced a decrease in concentration of motile spermatozoa to fewer than $5 \%$ of pre-treatment values (Mieusset and Bujan, 1994). Although significant increases in scrotal temperature appear to adversely affect male fertility, slight increases, such as 0.8 to $1.0^{\circ} \mathrm{C}$, appear to have no affect on spermatogenesis or spermatozoa function in the human male (Wang et al., 1997).

\subsection{Influence of Increased Ambient Temperature on Reproductive Parameters}

Numerous reports exist on the influence of increased ambient temperature on lab animal reproductive parameters. When exposed to a single episode of $43^{\circ} \mathrm{C}$ for $10 \mathrm{~min}$, mice experienced a decrease in testes weight $7 \mathrm{~d}$ post-exposure (Shilkina, 1976). Other 
studies have shown that DNA quality was reduced when mice were exposed to temperatures as low as $38^{\circ} \mathrm{C}$ (Sailer et al., 1997) and as high as $42^{\circ} \mathrm{C}$ for a period of 30 min (Perez-Crespo et al., 2008). Mice mated at predetermined intervals after heat treatment displayed subfertility consistent with damage to spermatocytes (Rockett et al., 2001), which is consistent with reports that damage to spermatocytes occurred within the testes at the time of heat stress (Perez-Crespo et al., 2008). Rabbits, a species extremely sensitive to heat stress, were exposed to an estimated temperature-humidity index (THI) value of 30.1 (very severe heat stress) for 3 mo, after which they presented significantly lower ejaculate volume, concentration of spermatozoa, and total sperm output; however, quality of spermatozoa remained unaffected (Marai et al., 2002). When compared to control group boars that were maintained at a temperature of $20^{\circ} \mathrm{C}$, boars exposed to ambient and radiant temperatures of $33^{\circ} \mathrm{C}$ for $72 \mathrm{~h}$ showed significant decreases in spermatozoal concentration, total motility of spermatozoa, and total spermatozoa per ejaculate, as well as an increase in primary and secondary abnormalities (McNitt and First, 1970). These results suggest that exposure to relatively low temperatures for an extended period of time may induce heat stress leading to fertility problems that can persist for 2 mo after initial exposure. Similarly, decreases in spermatozoal motility and percent normal spermatozoa was observed after boars were exposed to either $34.5^{\circ} \mathrm{C}$ for $8 \mathrm{~h} / \mathrm{d}$ or $31^{\circ} \mathrm{C}$ for $16 \mathrm{~h} / \mathrm{d}$ for $90 \mathrm{~d}$ (Wettemann et al., 1976). Pregnancy rates in gilts bred with semen from the heat stressed boars were significantly lower, as was embryonic survival at $30 \mathrm{~d}$ (Wettemann et al., 1976). Boars initially housed at a temperature of $20^{\circ} \mathrm{C}$ were subjected to an increase in air temperature of $1^{\circ} \mathrm{C} / \mathrm{d}$ for $20 \mathrm{~d}$, which elicited 
SST as high as $39^{\circ} \mathrm{C}$ (Stone, 1982). Seminal parameters were affected due to thermal insult to the testes, with a decrease in motility of spermatozoa observed when air temperature reached $30^{\circ} \mathrm{C}$ and a decrease in percent normal spermatozoa noted during the last week of heat treatment (Stone, 1982). These studies suggest that boars are more sensitive to lower ambient temperatures than other species, and heat stress is an extremely important factor to consider in their management (Konavongkrit et al., 2005). This information has prompted swine breeders to develop temperature management protocols to optimize the fertility of boars (Konavongkrit et al., 2005). Increased sensitivity to thermal insult may be due to the location of the testes close to the body wall, which differs from the more pendulous conformations of the scrota of stallions and bulls. The particularly pendulous structure of the bull scrotum allows the testes to remain farther from the body and to dissipate heat more efficiently. Nevertheless, when bulls were exposed to ambient temperatures higher than $29^{\circ} \mathrm{C}$, motility and concentration of spermatozoa decreased, as did total numbers of spermatozoa, for as long as 2 mo following heat stress (Casady et al., 1953).

\subsection{Influence of Exercise on Reproductive Parameters of Other Species}

Previous studies have been performed examining the reproductive parameters of men who exercise strenuously on a regular basis (Jung and Schuppe, 2007). An increase in spermatozoa morphological abnormalities was observed in endurance-trained cyclists (Gebreegziabher et al., 2004), while a decrease in spermatozoal concentration, ejaculate 
volume, and total number of spermatozoa was apparent in recreational male athletes subjected to more strenuous workouts (Vaamonde et al., 2006).

\subsection{Influence of Exercise on Stallion Reproductive Parameters}

A limited number of studies have explored the effects of thermal insult on stallion reproductive parameters, especially regarding thermal insult as a result of exercise, and results of these studies are conflicting. The first study of its kind, showed that daily minimal exercise by lunging at the walk and trot during a 16-wk period had no influence on semen quality (Dinger et al., 1986). However, the results were likely confounded by immaturity of the stallions as well as seasonal effects on spermatozoa production (Dinger et al., 1986). In contrast, Lange et al. (1997) recorded significantly better progressive spermatozoa motility in breeding stallions being trained and used in dressage and jumping competitions than in those used for breeding alone, although stallions were not blocked for age or other factors, and the extent of training was not defined. Results similar to those of Lange were obtained from a survey that found significantly higher fertility in intensively-trained Icelandic stallions when compared to moderately trained or untrained stallions (Davies Morel and Gunnarsson, 2000); however, again, intense versus moderate training was not clearly characterized. A more recent study with a better-defined exercise regimen consisting of repeated strenuous exercise (until exhaustion) on a high-speed treadmill $2 \mathrm{~d} / \mathrm{wk}$ for 4 wk was found to cause a distinct stress reaction in stallions that compromised the quality of fresh and frozenthawed semen during and up to 1 mo after exercise (Janett et al., 2006). Increases in the 
percentage of major spermatozoa defects immediately following the exercise period were observed, as well as increases in acrosome defects and nuclear vacuoles during the exercise period (Janett et al., 2006). Additionally, a decrease in motility of spermatozoa was observed 16 wk after the exercise period (Janett et al., 2006). Related research conducted by Staempfli et al. (2006) sought to determine how SST would be affected by treadmill or ridden exercise in stallions wearing a net-like testicular sling (suspensory) designed to hold the testicles close to the body during jumping, eventing, or fox hunting. The study showed that treadmill and ridden exercise significantly influenced SST, with levels being higher with suspensory than without, which was likely due to the impairment of airflow with suspensory (Staempfli et al., 2006). Also, SST of stallions subjected to treadmill exercise were significantly higher than those that were ridden, and scrotal cooling seemed to be directly related to gait in ridden horses, with a decrease in initial SST of approximately $3^{\circ} \mathrm{C}$ during cantering and an increase of more than $3^{\circ} \mathrm{C}$ from initial SST measurements during the walking cool-down (Staempfli et al., 2006). Semen characteristics were not evaluated during this study.

\subsection{Exercise and Related Increases in Core Body Temperatures in the Equine}

Numerous equine exercise physiology studies have documented the increase in body temperature that occurs when a horse is exercised. Several reports have recorded rectal temperatures in the range of 39.4 to $41.1^{\circ} \mathrm{C}$ for exercised horses, as well as persistent elevated temperatures during 30 min recovery periods (Webb et al., 1987;

Scott, 1992; Kohn et al., 1999). 


\subsection{Inferences}

Research examining the effects of exercise on male reproductive processes is questionable; however, increased thermal stress due to extreme ambient temperature or scrotal insulation clearly adversely affects reproductive processes in males of all species. Although literature has not conclusively elucidated the ambient temperatures at which spermatogenesis might be adversely affected, we can infer that animals dwelling in a hot and humid environment like south-central Texas may be more likely to suffer thermal testicular insult resulting in decreased reproductive parameters. Stallions being rigorously exercised during extreme temperatures may be at increased risk. If exercise can maintain a substantial increase in body temperature long enough to cause thermal insult to the testes, then many stallions could potentially suffer severe reproductive losses. High quality ejaculates are absolutely essential for success in artificial reproductive technologies such as $\mathrm{AI}$, which is becoming increasingly popular in the equine industry. If stallions do suffer reproductive losses preventing the use of AI, then substantial economic losses will ensue due to an increase in management costs, as well as loss of breeding fees and valuable male genetics. Research in other domestic livestock species has led to changes in management in order to minimize thermal stress. Further research is required to determine correlations between ambient, scrotal, and core body temperatures that are capable of causing impaired reproductive capacity so that stallion management practices can be improved in an effort to preserve the fertility of performance stallions, and thus their value as athletes and sires. 


\section{MATERIALS AND METHODS}

\subsection{Stallions, Housing, and Diets}

Eight mature Miniature Horse stallions (4 to $16 \mathrm{y}, 72.1$ to $110.7 \mathrm{~kg}$ ) were used in a 12 wk study from early July - early October (Table A.1). The stallions were brought to the Texas A\&M University Horse Center $90 \mathrm{~d}$ prior to the onset of the study and remained there throughout. Stallions were confined to individual stalls (1.8 $\mathrm{m} \mathrm{x} 1.8 \mathrm{~m})$ that allowed visual access to other horses and featured attached small dry paddock runs (7.3 $\mathrm{m} \times 1.8 \mathrm{~m})$ to allow each stallion free exercise every other day. Project approval was granted by the Texas A\&M University Institutional Agricultural Animal Care and Use Committee using guidelines set forth by the Federation of Animal Science Societies (1999).

Body weight (BW), rump fat (rf), and body condition scores (BCS) of each stallion were measured weekly to aid in diet determination, and percent extractable fat (EF) was determined as previously described (Westervelt et al., 1976). Each stallion was fed $12.5 \% \mathrm{CP}$ coastal hay (Table B.1) in amounts equal to $1.5 \%$ of his BW daily, and a $16.1 \% \mathrm{CP}$ concentrate (Table B.2) twice daily in amounts consistent with each stallion's body condition (Henneke et al., 1983; Westervelt et al., 1976). Each stallion was allowed free access to fresh water. Table 1 lists mean \pm SD values for each measurement of body condition at the onset ( Wk 0) and the end (Wk 12) of the project. 
TABLE 1. Mean ( \pm SD) body condition endpoints of non-exercised (non-ex; control) and exercised (ex) stallions at the onset (Week 0) and at the end (Week 12) of the study ( $n=4$ /group).

\begin{tabular}{lcccc}
\hline & \multicolumn{2}{c}{ WEEK 0 } & \multicolumn{2}{c}{ WEEK 12 } \\
& NON-EX & EX & NON-EX & EX \\
\hline Body Weight $(\mathrm{kg})$ & $87.09 \pm 10.27$ & $95.82 \pm 15.93$ & $93.67 \pm 8.16$ & $98.32 \pm 0.01$ \\
Body Condition Score & $4.75 \pm 0.87$ & $5.20 \pm 0.24$ & $5.13 \pm 0.63$ & $5.38 \pm 0.29$ \\
Rump Fat Thickness $(\mathrm{cm})$ & $0.35 \pm 0.17$ & $0.58 \pm 0.38$ & $0.34 \pm 0.13$ & $0.54 \pm 0.29$ \\
Extractable Fat $(\%)$ & $5.78 \pm 0.95$ & $7.07 \pm 2.15$ & $5.71 \pm 0.73$ & $6.82 \pm 0.29$ \\
\hline
\end{tabular}

\subsection{Initial Rest/Acclimation Period}

The stallions were allowed an initial 90-d rest period, during which they were stalled and only allowed free exercise every other day. This time period was also used to acclimatize the stallions to the breeding barn and the procedures involved with semen collection. Each stallion was trained to mount a breeding phantom and ejaculate into an artificial vagina $(\mathbf{A V})$.

\subsection{Thermal Sensor Implants}

Two subdermal thermal sensory devices (Digital Angel Corp., St. Paul, MN) were surgically implanted into each stallion by a veterinary surgeon. Prior to surgery, a intramuscular (IM) tetanus toxoid $(1 \mathrm{~mL})$ was administered in addition to intravenous (IV) flunixin meglumine $(1 \mathrm{mg} / \mathrm{kg})$ to control inflammation and analgesia. Xylazine (1 $\mathrm{mg} / \mathrm{kg}, \mathrm{IV})$ was administered as a sedative prior to induction with ketamine $(2 \mathrm{mg} / \mathrm{kg}$, IV), and stallions were placed in dorsal recumbency. A $10 \times 10 \mathrm{~cm}$ area on the left of the neck over the trapezius muscle was clipped, and the neck area and scrotum were 
aseptically prepared for surgery with a betadine and alcohol scrub. A sterile, prepackaged telemetric probe was preloaded into a syringe with a $12-\mathrm{g}$ needle. The most ventral portion of the scrotum was grasped and the vaginal tunic identified within the scrotum. By tenting the skin of the scrotum, the needle was inserted past the skin into the vaginal tunic, and the plunger was deployed. The skin surrounding the needle was tightly grasped as the needle was withdrawn in order to avoid removing the transponder with the needle. Transponder placement in the scrotum was verified by manual palpation. A second transponder was placed beneath the skin and subcutaneous tissue into the trapezius muscle on the left side of the neck using a similar technique. No closure of skin was needed at either site. Scanners (Pocket Reader EX ${ }^{\mathrm{TM}}$, Digital Angel Corp., St. Paul, MN) were used to read internal temperatures measured by the implanted microchips during the study.

\subsection{Predicted Daily Spermatozoa Output}

Testicular volume was computed from ultrasonographic measurements of width, height, and length of the testes. Predicted daily spermatozoa output (DSO) was calculated using a regression formula, as previously reported (Love et al., 1991) (Table 2). 
TABLE 2. Predicted daily spermatozoa output (DSO; in billions) for all stallions.

\begin{tabular}{ll}
\hline \hline STALLION & DSO \\
\hline Bon Jovi & 0.88 \\
Rock & 0.88 \\
Rockstar & 0.57 \\
Scooter & 0.87 \\
Beatle & 0.55 \\
Brave & 1.13 \\
Eagle & 1.07 \\
Mick Jagger & 1.63 \\
\hline
\end{tabular}

\subsection{Blocking and Treatment Groups}

Ejaculates from each stallion were collected each day for 5 consecutive days, which is sufficient for miniature stallions, although standard protocol for full-sized stallions requires 10 consecutive collections. The mean value for sperm output during the final $2 \mathrm{~d}$ of semen collection was used to represent actual DSO, as the numbers of spermatozoa no longer varied significantly (Gebauer et al., 1974; Thompson et al., 2004). No semen was collected for at least $7 \mathrm{~d}$ prior to each DSO determination.

Baseline DSO parameters for each stallion were determined at $0 \mathrm{wk}$.

Subsequently, stallions were blocked by age, testicular volume (TV), total number of spermatozoa/ejaculate, and percent normal spermatozoa before being randomly assigned to either a non-exercised (non-ex; control) or exercised (ex; treatment) group (Table 3). 
TABLE 3. Mean ( \pm SD) values of blocking criteria used to assign stallions to non-ex (control) or ex (treatment) groups ( $\mathrm{n}=4$ /group).

\begin{tabular}{lcc}
\hline \hline & NON-EX & EX \\
\hline Age $(\mathrm{yr})$ & $8.25 \pm 4.35$ & $9 \pm 5.10$ \\
TV $(\mathrm{mL})$ & $67.5 \pm 9.81$ & $74.25 \pm 18.84$ \\
Total \# Cells $\left.(\mathrm{x} \mathrm{10})^{9}\right)$ & $1.217 \pm 0.34$ & $1.684 \pm 1.057$ \\
Normal Cells $(\%)$ & $63.75 \pm 17.50$ & $70 \pm 8.98$ \\
\hline
\end{tabular}

\subsection{Fitness Evaluation - Standard Exercise Tests}

A high speed, motorized equine treadmill was used to complete a standard exercise test (SET) on each stallion before (pre-EP) and after (post-EP) the exercise period (EP) in order to determine whether exercised stallions achieved a higher level of fitness as a result of the exercise protocol prescribed during the study. Prior to the SET, each stallion was fitted with a Polar Equine WearLink ${ }^{\mathrm{TM}}$ heart rate monitor (Polar Electro Oy, HQ, Finland). Resting heart rate (HR), respiration rate (RR), subcutaneous neck temperature (SQNT), RCT, SQST, AMBT, and relative ambient humidity (HUM) were recorded. The 28-min SET protocol is listed in Table 4. Heart rate was recorded at $5,10,15,20$, and 25 min during exercise. Upon completion of the SET at $28 \mathrm{~min}, \mathrm{HR}$, RR, and all temperatures (SQNT, RCT, and SQST) were recorded. The HR monitor remained on each stallion during a 10 min hand-walking cool-down to record the HR, $\mathrm{RR}$, and temperatures (SQNT, RCT, and SQST) at 10 min post-SET. The monitor was then removed, and the stallion was tied in a stall for $20 \mathrm{~min}$. All temperatures (SQNT, RCT, and SQST) were recorded at 30 min post-SET. Additionally, blood was collected from each stallion immediately before and after SET via jugular venipuncture with 
lithium heparinized vacutainer tubes (BD Vacutainer ${ }^{\circledR}$, Franklin Lakes, NJ). Tubes of blood were immediately placed in a $16^{\circ} \mathrm{C}$ cooler (Koolatron PC3, Koolatron ${ }^{\mathrm{TM}}$, Brantford, Ontario) until delivery to the Clinical Pathology Laboratory at the Texas A\&M University Veterinary Medical Teaching Hospital within 4 h of collection, where blood lactate concentrations were analyzed.

TABLE 4. Standard exercise test (SET) protocol for all stallions; used before (pre-EP) and after (post-EP) the exercise period (EP).

\begin{tabular}{c|c|c}
\hline \hline TIME $(\min )$ & VELOCITY $(\mathrm{m} / \mathrm{s})$ & GRADE $(\%)$ \\
\hline 0 & 1.8 & 0 \\
5 & 2.4 & 0 \\
10 & 2.4 & 2.1 \\
15 & 4 & 2.1 \\
20 & 4.5 & 2.8 \\
25 & 5.5 & 2.8 \\
28 & & \\
\hline
\end{tabular}

\subsection{Exercise}

During a 12-wk period (between DSO determinations at wk 0, 4, 8, and 12), ex stallions $(n=4)$ were exercised on a high-speed motorized equine exerciser. Exercise protocol was divided into 3 phases: Phase I ( 8 min walking warm-up), Phase II (15 min long trot/canter), and Phase III (8 min walking cool-down) (Table 5). Exercise began at $1200 \mathrm{~h}$ on each day of exercise. A target heart rate of 150 to 160 beats per minute (bpm) was achieved and maintained during Phase II. Temperatures (SQST, SQNT, and RCT) were recorded before exercise, immediately after Phase II, immediately after 
Phase III, and at 60 and 120 min post-exercise. Ambient temperatures were recorded

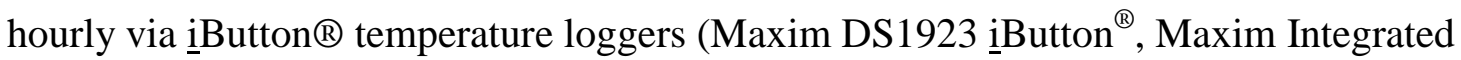
Products, Inc., Sunnyvale, CA) located in the shaded stall area (where pre- and postexercise temperatures were measured) and in the sun near the motorized equine exerciser (where Phase II and III temperatures were measured). Hourly HUM percentages were obtained (NOAA, 2010) and used to calculate the hourly THI experienced during exercise periods. Non-exercised stallions were tied outside while the ex stallions were being exercised, which subjected both groups to consistent ambient temperatures.

TABLE 5. Exercise protocol ( $4 \mathrm{~d} / \mathrm{wk}$ ) used for ex (treatment) stallions during a $12-\mathrm{wk}$ exercise period (EP) with temperatures recorded at each time point.

\begin{tabular}{ccccc}
\hline TIME POINTS & DESCRIPTION & DURATION & INTENSITY & LOCATION \\
\hline 0 & Resting & & & Shade \\
& Phase I & $8 \mathrm{~min}$ & Walk & Sun \\
22 & Phase II & $15 \mathrm{~min}$ & Long trot/Canter & Sun \\
30 & Phase III & $7 \mathrm{~min}$ & Walk & Sun \\
60 min-post & Recovery & $60 \mathrm{~min}$ & & Shade \\
120 min-post & Recovery & $60 \mathrm{~min}$ & & Shade \\
\hline
\end{tabular}

${ }^{\mathrm{a}}$ Exercise began at $1200 \mathrm{~h}$ on each day of exercise

\subsection{Semen Collection}

Throughout the study, 4 DSO determinations were completed for each stallion at 0, 4, 8, and 12 wk, with 0 wk representing baseline values. Ejaculates were collected at the Texas A\&M University Horse Center Breeding Farm facilities using a $33 \mathrm{~cm}$ Missouri-model AV (Nasco, Ft. Atkinson, WI). Each AV was lubricated thoroughly 
with Priority Care Lube ${ }^{\circledR}$ (Nasco, Ft. Atkinson, WI) and then filled with 48.8 to $54.4^{\circ} \mathrm{C}$ water, depending on stallion preference. All collection materials were kept in an

incubator at $37^{\circ} \mathrm{C}$. A sterilized liner (Playtex ${ }^{\circledR}$ Drop-Ins ${ }^{\circledR}$, Neenah, WI) was placed inside of a collection container with a micromesh semen filter stretched across the opening to filter gel and debris from the semen sample. Gel was discarded upon semen collection, and the sample was taken immediately to the laboratory and placed into a $37^{\circ} \mathrm{C}$ incubator.

\subsection{Semen Processing}

Equipment used for semen analysis during this study was maintained at the Texas A\&M School of Veterinary Medicine in the Theriogenology Laboratory, which was located approximately $2 \mathrm{~km}(5 \mathrm{~min})$ from the Texas A\&M University Horse Center. Therefore, semen was initially processed at the Horse Center and had to be transported to the Theriogenology Laboratory for analysis. For short-term storage, semen may be cooled to room temperature $\left(20^{\circ} \mathrm{C}\right)$ in an effort to reduce the metabolic rate of spermatozoa, thus preventing a decline in viability and motility (Davies Morel, 2008). Additionally, spermatozoa are susceptible to ultraviolet light (Davies Morel, 2008). Therefore, during the present study, semen samples were placed in a placed in a $16^{\circ} \mathrm{C}$ cooler (Koolatron ${ }^{\mathrm{TM}} \mathrm{PC} 3$, Koolatron ${ }^{\mathrm{TM}}$, Brantford, Ontario), which allowed for maintenance of the samples at a temperature of approximately $20^{\circ} \mathrm{C}$ in an environment free from ultraviolet light. 
Gel-free volume (VOL) of each ejaculate was measured in the Horse Center Laboratory using a $10-\mathrm{mL}$ graduated cylinder. Two $0.5-\mathrm{mL}$ aliquots of raw semen were prepared; one aliquot was placed in a $16^{\circ} \mathrm{C}$ cooler until analysis for concentration and viability, and the second aliquot was frozen in liquid nitrogen and maintained at $-80^{\circ} \mathrm{C}$ for later assessment of DNA quality using the Sperm Chromatin Structure Assay (SCSA). Raw semen was also diluted in buffered formol saline for pending morphological analysis. Three additional $1.5-\mathrm{mL}$ aliquots were prepared using the remaining fresh semen diluted with a commercial semen extender (INRA-96, Breeder's Choice, Aubrey, TX) at a 1:4 dilution. One aliquot was placed in the $16^{\circ} \mathrm{C}$ cooler for later analysis of motion characteristics, and the other two aliquots were stored in separate cooling devices (Equitainer-11, Hamilton-Thorne Biosciences, Beverly, MA), with one sample analyzed after $24 \mathrm{~h}$ and the other after $48 \mathrm{~h}$ of storage. All the materials used in processing ejaculates were maintained at $37^{\circ} \mathrm{C}$ in an incubator until time of use. The $16^{\circ} \mathrm{C}$ cooler, containing a $0.5-\mathrm{mL}$ aliquot of raw semen and a $1.5-\mathrm{mL}$ aliquot of extended semen, was transported to the Theriogenology Laboratory for analysis within 30 min of collection.

\subsection{Semen Analysis}

Concentration of Spermatozoa. Upon arrival at the Theriogenology Laboratory, fresh (T0) samples were removed from the $16^{\circ} \mathrm{C}$ cooler. Raw semen was analyzed for fluorescence-based spermatozoa concentration analysis (CONC) and membrane integrity (VIAB) (NucleoCounter SP-100; ChemoMetec, Allerød, Denmark). 
Spermatozoal Motility. Extended semen was analyzed for percentage of total motile (TMOT) and progressively motile (PMOT) spermatozoa via computer-assisted spermatozoa motion analysis (CASMA, IVOS version 12.2 L, Hamilton-Thorne Biosciences, Beverly, MA). Prior to analysis, extended semen samples were further diluted with INRA-96® extender to achieve a concentration of 25 to $30 \times 10^{6}$ spermatozoa/mL. Samples were then placed in a $37^{\circ} \mathrm{C}$ incubator for $15 \mathrm{~min}$ prior to motility analysis (Jasko et al., 1992). Warmed $\left(37^{\circ} \mathrm{C}\right)$ analysis chambers (fixed height of $20 \mu \mathrm{m}$ ) affixed to microscope slides (Leja Standard Count 2 Chamber slides; Leja Products, B.V., Nieuw-Vennep, The Netherlands) were loaded with a $6-\mu \mathrm{L}$ volume of extended semen and inserted into the CASMA instrument for evaluation. A total of 10 microscopic fields and a minimum of 500 spermatozoa were examined per sample. Settings for the IVOS system included the following: frames acquired-45; frame rate-60 Hz; minimum contrast-70; minimum cell size-4 pixels; minimum static contrast-30; straightness threshold for progressive motility-50\%; average-path velocity (VAP) threshold for PMOT-30 $\mu / \mathrm{sec}$, VAP threshold for static cells $<15 \mu /$ sec; cell intensity106 ; static head size- 0.60 to 2.00 ; static head intensity, -0.20 to 2.01 ; static elongation-40 to 85 ; LED illumination intensity-2200.

Spermatozoal Morphology. Fresh semen samples preserved in 10\% buffered formol saline were evaluated using a wet mount preparation and differential interference contrast microscopy (Olympus BX60, Olympus America, Inc., Melville, NY, 1250X magnification). Specifically, 1 to $2-\mu 1$ drop was placed under a coverslip, and a total of 100 cells were counted per sample. The percent of morphologically normal sperm 
(NORMCELL) were identified, as well as specific morphologic abnormalities including abnormal heads (AH), acrosomes (AA), and midpieces (AM), detached heads (DH), bent tails (BT), coiled tails (CT), and premature germ cells (PGC) (Kenney et al., 1983). Percentages of each abnormality were determined by counting all abnormalities present on a sperm.

Sperm Chromatin Quality. Quality of DNA was assessed using the SCSA as previously described (Love and Kenney, 1998). Samples were thawed in a $37^{\circ} \mathrm{C}$ water bath. Aliquots (approximately $5 \mu \mathrm{L}$ ) of thawed semen were mixed with $195 \mu \mathrm{L}$ of a TNE buffer solution (500 mL mixture of $0.79 \mathrm{~g}$ Trizma $\mathrm{HCl}, 4.38 \mathrm{~g} \mathrm{NaCl}$, and $0.186 \mathrm{~g}$ EDTA), which was then combined with $400 \mu \mathrm{L}$ of a low $\mathrm{pH}(\sim 1.2)$ Triton $\mathrm{X}-100$ detergent solution $(500 \mathrm{~mL}$ mixture of $4.38 \mathrm{~g} \mathrm{NaCl}, 20 \mathrm{~mL} 2 \mathrm{~N} \mathrm{HCl}$, and $0.5 \mathrm{~mL}$ Triton$\mathrm{X}$ detergent) for $30 \mathrm{sec}$. A solution of the heterochromatic dye, acridine orange, was added $(1.2 \mathrm{~mL}$ at $4.0 \mu \mathrm{g} / \mathrm{mL})$ to the sample, and it was processed within $30 \mathrm{sec}$ on a flow cytometer (FACScan, Becton Dickinson, Mountain View, CA). Quantification of DNA denaturation in each cell was determined by the term alpha-t $(\alpha t)$, defined as the ratio of $\mathrm{red} /(\mathrm{red}+$ green fluorescence). The alpha-t $(\alpha \mathrm{t})$ designation is used to describe the relationship between the amounts of green (native, double-stranded DNA) and red (single-stranded DNA) fluorescence. The endpoint evaluated was the percent of cells with abnormal DNA, or the cells outside the main population (COMPat). This was determined by selecting those sperm cells to the right of the main population and represents the number of sperm cells outside the main population as a percentage of the total number of sperm cells evaluated. 
Cooled Samples. Samples stored for 24 h (T24) and 48 h (T48) were evaluated for concentration and viability of spermatozoa, TMOT, PMOT, and DNA quality.

\subsection{Statistical Analysis}

Spermatozoa and SET data were normally distributed; therefore, they were subjected to parametric analysis by repeated measures in time using PROC MIXED (SAS v 9.1; SAS Inst. Inc., Cary, NC). The model included group (non-ex or ex), wk (0, 4, 8, or 12), semen collection sample (T0, T24, and T48), and all interactions, with stallion as the subject of the repeated measures.

Temperature data were analyzed using a nonparametric test that made no assumptions based on distribution of the data, as the data were not normally distributed. A Spearman's Rank Correlation test was performed to determine relationships between SQST, SQNT, RCT, AMBT, HUM, and THI during exercise. 


\section{RESULTS}

\subsection{Implantation of Subcutaneous Scrotal Devices}

No fever, edema, or other deleterious effects were noted from the subdermal implantation of thermal sensory devices into the necks or scrotums of the stallions in this study.

\subsection{Ambient Temperature, Humidity, and Temperature-Heat Index}

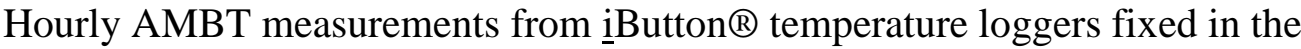
stall area (shade) and exercise area (sun) did not differ among weeks of the study; however, a difference among time points $(\mathrm{P}<0.0001)$ was observed (Fig. 1). The increase in AMBT at 22 and 30 min were recorded during Phases I-III of exercise in the heat of the sun, while 0 min, 60 min-post, and 120 min-post were recorded in the shaded stall area. A steady decrease in HUM was observed during exercise time points but was not significant. Ambient temperature and HUM were used to calculate THI, which did not differ among weeks, but did increase at 22 and 30 min of exercise $(\mathrm{P}<0.0001)$ (Fig. 1). Mean AMBT, HUM, and THI for each time point during exercise are listed in Table 6. 


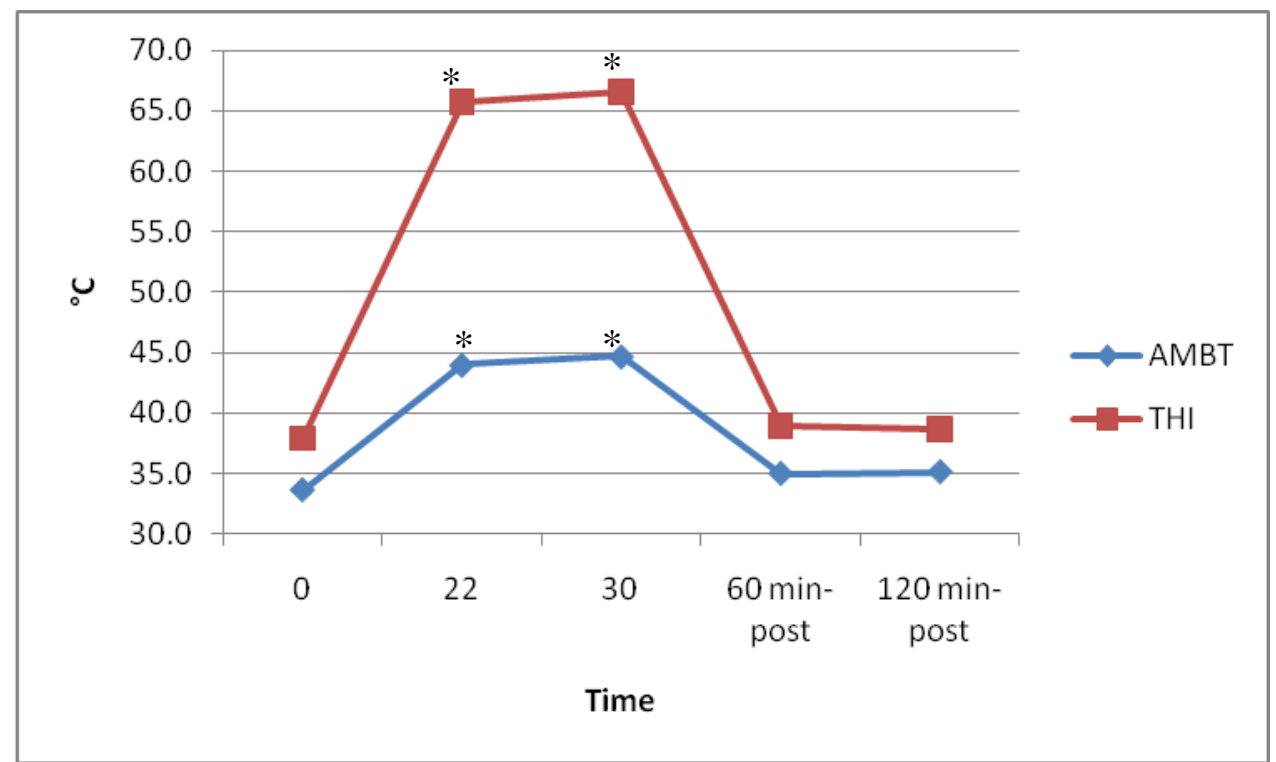

FIGURE 1. Mean ambient (AMBT) and temperature-heat index (THI) to which all stallions were subjected at each time point during exercise protocol.

* Indicates significant increase from other time points along the same line $(\mathrm{P}<0.0001)$

TABLE 6. Mean ambient (AMBT), humidity (HUM), and temperature-heat index (THI) at each time point during exercise.

\begin{tabular}{lccccc}
\hline \hline & 0 & 22 & 30 & 60 min-post & 120 min-post \\
\cline { 2 - 6 } AMBT $\left({ }^{\circ} \mathrm{C}\right)$ & 33.6 & $44.0^{*}$ & $44.7^{*}$ & 35.0 & 35.1 \\
HUM $(\%)$ & 51.3 & 47.6 & 46.7 & 45.9 & 44.0 \\
THI $\left({ }^{\circ} \mathrm{C}\right)$ & 37.9 & $65.7^{*}$ & $66.5^{*}$ & 38.9 & 38.7 \\
\hline
\end{tabular}

* Indicates significant increase from other time points $(\mathrm{P}<0.0001)$

\subsection{Changes in Stallion Temperatures Due to Exercise}

During exercise protocol, ex stallions achieved a mean average HR of $149 \mathrm{bpm}$ with a mean peak value of $164 \mathrm{bpm}$. Temperatures (RCT, SQNT, and SQST) during exercise were significantly higher $(\mathrm{P}<0.0002)$ in both ex and non-ex stallions at time 
points 22 and $30 \mathrm{~min}$ compared to other times ( $0 \mathrm{~min}, 60 \mathrm{~min}$-post, and $120 \mathrm{~min}$-post; Tables 7 to 9$)$. Rectal and SQNT of ex stallions increased $(\mathrm{P}<0.0001)$ by $1.9^{\circ} \mathrm{C}$ and $2.4^{\circ} \mathrm{C}$, respectively, at $22 \mathrm{~min}$ of exercise (Fig. 2). Although not significant $(\mathrm{P}>0.05)$, a mean increase in SQST of $0.8^{\circ} \mathrm{C}$ from was achieved from 0 to 22 min of exercise (Fig. 3). Changes in temperature of individual stallions within groups can be viewed in Tables C.1 and C.2.

TABLE 7. Mean $( \pm \mathrm{SD})$ rectal temperatures $(\mathrm{RCT})$ of non-ex (control) and ex (treatment) stallions during exercise protocol ( $n=4$ /group).

\begin{tabular}{|c|c|c|c|c|c|}
\hline \multirow{3}{*}{$\frac{\text { TIME }}{0}$} & \multicolumn{5}{|c|}{ RCT } \\
\hline & \multicolumn{2}{|r|}{ NON-EX } & \multicolumn{3}{|c|}{ EX } \\
\hline & 37.60 & $\pm 0.18^{\mathrm{ac}}$ & 37.30 & \pm & $0.15^{\mathrm{ac}}$ \\
\hline 22 & 37.78 & $\pm 0.24^{\mathrm{ac}}$ & 39.22 & \pm & $0.43^{\mathrm{bd}}$ \\
\hline 30 & 37.82 & $\pm \quad 0.20^{\mathrm{ac}}$ & 39.21 & \pm & $0.47^{\text {bd }}$ \\
\hline 60 min-post & 37.56 & $\pm 0.17^{\mathrm{ac}}$ & 37.44 & \pm & $0.14^{\mathrm{ac}}$ \\
\hline 120 min-post & 37.41 & $\pm 0.17^{\mathrm{ac}}$ & 37.23 & \pm & $0.19^{\mathrm{ac}}$ \\
\hline
\end{tabular}

${ }^{\mathrm{ab}}$ Means not sharing the same superscript within a column are different $(\mathrm{P}<0.03)$
${ }^{\mathrm{cd}}$ Means not sharing the same superscript within a row are different $(\mathrm{P}<0.03)$ 
TABLE 8. Mean ( \pm SD) subcutaneous neck temperatures (SQNT) of non-ex (control) and ex (treatment) stallions during exercise protocol ( $\mathrm{n}=4 /$ group).

\begin{tabular}{llllll}
\hline \hline & \multicolumn{5}{c}{ SQNT } \\
\cline { 2 - 5 } TIME & \multicolumn{3}{c}{ NON-EX } & EX \\
\hline 0 & 37.72 & \pm & $0.23^{\mathrm{ac}}$ & 37.46 & $\pm 0.23^{\mathrm{ac}}$ \\
22 & 38.03 & \pm & $0.32^{\mathrm{ac}}$ & 39.94 & $\pm 0.98^{\mathrm{bd}}$ \\
30 & 38.01 & \pm & $0.36^{\mathrm{ac}}$ & $39.71 \quad \pm 0.90^{\mathrm{bd}}$ \\
60 min-post & 37.66 & \pm & $0.31^{\mathrm{ac}}$ & $37.55 \quad \pm 0.23^{\mathrm{ac}}$ \\
120 min-post & 37.54 & \pm & $0.25^{\mathrm{ac}}$ & $37.46 \pm 0.06^{\mathrm{ac}}$ \\
\hline not sharing the same superscript within a column are different $(\mathrm{P}<0.0001)$
\end{tabular}

TABLE 9. Mean ( \pm SD) subcutaneous scrotal temperatures (SQST) of non-ex (control) and ex (treatment) stallions during exercise protocol ( $\mathrm{n}=4$ /group).

\begin{tabular}{lllll}
\hline \hline & \multicolumn{4}{c}{ SQST } \\
\cline { 2 - 4 } TIME & \multicolumn{3}{c}{ NON-EX } & EX \\
\hline 0 & $33.80 \pm 0.23^{\mathrm{a}}$ & $33.91 \pm 1.02^{\mathrm{a}}$ \\
22 & $34.23 \pm 0.50^{\mathrm{a}}$ & $34.68 \pm 0.92^{\mathrm{b}}$ \\
30 & $34.31 \pm 0.44^{\mathrm{a}}$ & $34.66 \pm 0.99^{\mathrm{b}}$ \\
60 min-post & $33.83 \pm 0.24^{\mathrm{a}}$ & $33.69 \pm 0.24^{\mathrm{a}}$ \\
120 min-post & $33.97 \pm 0.26^{\mathrm{a}}$ & $33.59 \pm 0.11^{\mathrm{a}}$ \\
\hline
\end{tabular}

${ }^{\mathrm{ab}}$ Means not sharing the same superscript within a column are different $(\mathrm{P}<0.03)$

Means across rows are not different $(\mathrm{P}>0.05)$ 


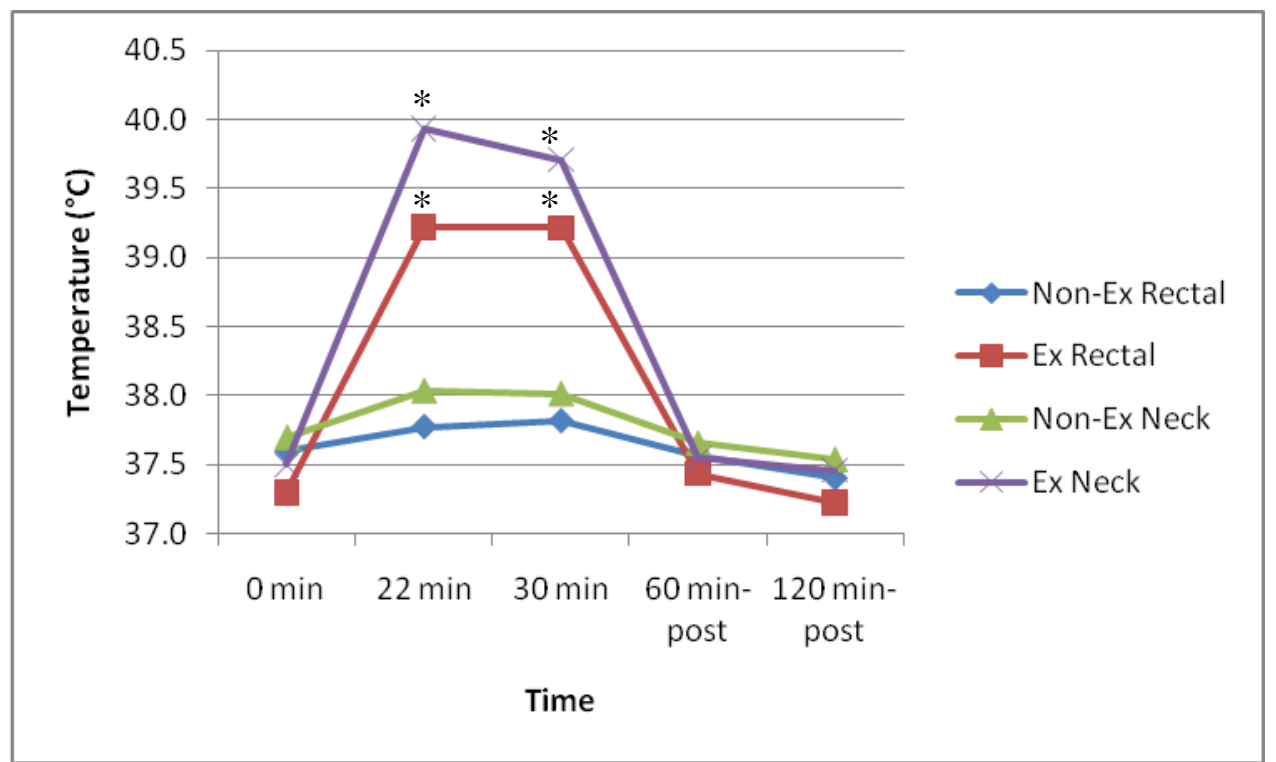

FIGURE 2. Mean rectal and neck temperatures for non-ex (control) and ex (treatment) stallions during the exercise period ( $\mathrm{n}=4$ /group).

*Indicates significant increase from like time points along the same line $(\mathrm{P}<0.0001)$

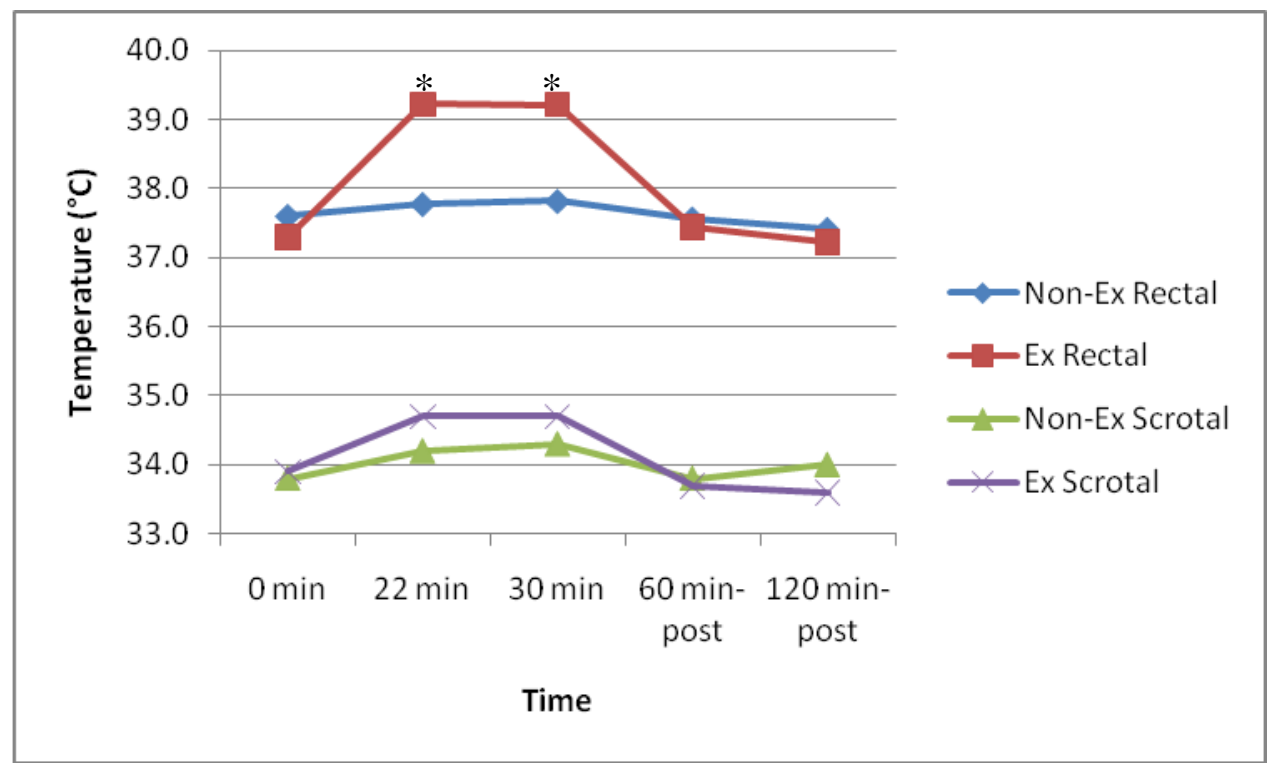

FIGURE 3. Mean rectal and scrotal temperatures for non-ex (control) and ex (treatment) stallions during the exercise period ( $\mathrm{n}=4$ /group).

*Signifies significant increase from like time points along the same line $(\mathrm{P}<0.0001)$ 


\subsection{Correlation Analysis between Different Temperature Recordings}

Significant, positive correlations existed between SQST, SQNT, RCT, AMBT, and THI (Table 10). Humidity showed no significant relationships with any of the measured temperatures in this study.

\subsection{Fresh Semen}

Semen parameter measurements from the final $2 \mathrm{~d}$ of each DSO determination were averaged to determine mean values of fresh semen for wk 0, 4, 8, and 12 (Table 11). No effects of treatment by time were observed for any of the endpoints measured.

TABLE 10. Mean correlations $\left(r_{s}\right)$ of subcutaneous scrotal temperature (SQST), subcutaneous neck temperature (SQNT), rectal temperature (RCT), ambient temperature (AMBT), humidity (HUM), and temperature-heat index (THI).

\begin{tabular}{c|ccc}
\hline \hline \multirow{2}{*}{ SQNT } & SQST & SQNT & RCT \\
\hline RCT & $0.476^{*}$ & & \\
AMBT & $0.761^{*}$ & $0.453^{*}$ & \\
HUM & $0.625^{*}$ & $0.513^{*}$ & $0.558^{*}$ \\
THI & $-0.074^{*}$ & $-0.263^{*}$ & -0.025 \\
\hline
\end{tabular}

* indicates $\mathrm{P}<0.0001$ 
TABLE 11. Mean ( \pm SD) semen characteristics for all samples (T0, T24, and T48) of non-ex (control) and ex (treatment) stallions ( $\mathrm{n}=4$ /group).

\begin{tabular}{|c|c|c|c|c|c|c|c|c|}
\hline & \multicolumn{2}{|c|}{ WEEK 0} & \multicolumn{2}{|c|}{ WEEK 4} & \multicolumn{2}{|c|}{ WEEK 8} & \multicolumn{2}{|c|}{ WEEK 12} \\
\hline & NON-EX & EX & NON-EX & $\mathbf{E X}$ & NON-EX & EX & NON-EX & EX \\
\hline \multicolumn{9}{|c|}{ VOLUME (mL) } \\
\hline T0 & $9.1 \pm 4.1$ & $9.1 \pm 4.3$ & $12.0 \pm 7.7$ & $7.7 \pm 4.8$ & $11.8 \pm 5.2$ & $11.9 \pm 2.6$ & $7.9 \pm 5.5$ & $10.1 \pm 8.2$ \\
\hline \multicolumn{9}{|c|}{ CONCENTRATION (million/mL) } \\
\hline T0 & $139.9 \pm 35.5$ & $193.5 \pm 39.0$ & $172.1 \pm 29.6$ & $203.8 \pm 51.3$ & $100.5 \pm 24.1$ & $169.8 \pm 35.1$ & $102.6 \pm 61.0$ & $218.1 \pm 127.7$ \\
\hline \multicolumn{9}{|c|}{ TOTAL CELLS (million spermatozoa) } \\
\hline T0 & $1217 \pm 339.7$ & $1683.9 \pm 1057.2$ & $1676.6 \pm 1215.5$ & $1466.3 \pm 808.0$ & $1100.0 \pm 628.1$ & $1908.0 \pm 899.5$ & $667.6 \pm 422.4$ & $1573.4 \pm 592.9$ \\
\hline \multicolumn{9}{|c|}{ NORMAL CELLS (\%) } \\
\hline T0 & $61.0 \pm 17.5$ & $72.5 \pm 5.8$ & $58.8 \pm 16.0$ & $74.5 \pm 3.7$ & $51.8 \pm 7.3$ & $73.3 \pm 6.1$ & $57.8 \pm 4.6$ & $71.3 \pm 6.5$ \\
\hline \multicolumn{9}{|c|}{ VIABILITY (\%) } \\
\hline T0 & $72.6 \pm 2.4$ & $72.9 \pm 5.5$ & $72.1 \pm 1.9$ & $75.8 \pm 4.1$ & $62.1 \pm 11.3$ & $72.6 \pm 5.7$ & $67.0 \pm 12.8$ & $76.1 \pm 7.2$ \\
\hline $\mathbf{T 2 4}$ & $66.1 \pm 9.0$ & $69.0 \pm 3.1$ & $64.6 \pm 6.2$ & $70.6 \pm 5.5$ & $66.4 \pm 2.9$ & $65.1 \pm 6.8$ & $68.5 \pm 6.9$ & $77.9 \pm 5.4$ \\
\hline T48 & $58.8 \pm 13.8$ & $66.3 \pm 2.4$ & $64.4 \pm 11.5$ & $70.8 \pm 5.1$ & $59.4 \pm 5.0$ & $68.6 \pm 7.4$ & $74.0 \pm 5.7$ & $79.8 \pm 2.4$ \\
\hline \multicolumn{9}{|c|}{ TOTAL MOTILITY (\%) } \\
\hline T0 & $71.8 \pm 7.1$ & $75.8 \pm 6.1$ & $71.5 \pm 5.8$ & $77.5 \pm 4.7$ & $63.3 \pm 5.9$ & $70.1 \pm 3.8$ & $58.3 \pm 15.4$ & $76.0 \pm 6.8$ \\
\hline T24 & $68.6 \pm 12.6$ & $73.4 \pm 9.9$ & $67.4 \pm 12.7$ & $74.9 \pm 9.9$ & $55.5 \pm 8.2$ & $65.9 \pm 4.6$ & $49.6 \pm 12.7$ & $64.1 \pm 9.2$ \\
\hline T48 & $49.9 \pm 13.4$ & $56.3 \pm 11.0$ & $44.1 \pm 20.7$ & $56.6 \pm 15.0$ & $35.3 \pm 8.1$ & $47.6 \pm 14.7$ & $40.1 \pm 14.3$ & $56.3 \pm 13.9$ \\
\hline \multicolumn{9}{|c|}{ PROGRESSIVE MOTILITY (\%) } \\
\hline T0 & $48.4 \pm 10.7$ & $52.9 \pm 8.8$ & $46.1 \pm 3.6$ & $53.5 \pm 8.7$ & $36.8 \pm 7.2$ & $46.3 \pm 9.1$ & $35.6 \pm 10.9$ & $55.1 \pm 8.5$ \\
\hline T24 & $37.8 \pm 14.2$ & $50.3 \pm 11.8$ & $39.4 \pm 15.3$ & $50.4 \pm 13.4$ & $26.6 \pm 8.4$ & $42.9 \pm 6.4$ & $23.1 \pm 10.7$ & $34.6 \pm 13.6$ \\
\hline T48 & $24.4 \pm 14.7$ & $31.5 \pm 10.7$ & $23.5 \pm 15.3$ & $34.4 \pm 15.4$ & $11.6 \pm 6.1$ & $27.5 \pm 12.7$ & $17.5 \pm 7.1$ & $32.6 \pm 13.0$ \\
\hline \multicolumn{9}{|c|}{ \% COMP-at } \\
\hline T0 & $12.7 \pm 8.3$ & $10.8 \pm 6.7$ & $13.3 \pm 6.3$ & $8.3 \pm 0.8$ & $12.8 \pm 3.3$ & $11.2 \pm 6.4$ & $12.1 \pm 4.5$ & $8.8 \pm 2.9$ \\
\hline T24 & $20.9 \pm 11.2$ & $16.0 \pm 5.1$ & $20.4 \pm 8.3$ & $13.9 \pm 3.7$ & $21.9 \pm 3.7$ & $16.9 \pm 5.2$ & $19.6 \pm 3.9$ & $15.1 \pm 4.0$ \\
\hline T48 & $22.7 \pm 13.5$ & $18.0 \pm 3.2$ & $22.9 \pm 7.0$ & $13.8 \pm 1.4$ & $25.2 \pm 4.4$ & $18.0 \pm 4.4$ & $22.3 \pm 3.4$ & $16.9 \pm 4.3$ \\
\hline
\end{tabular}

*No significant differences were observed $(\mathrm{P}>0.05)$ 
No significant difference was observed in gel-free semen volume or spermatozoal concentration between groups (Figures D.1 and D.2, respectively). Total spermatozoal number was calculated by multiplying semen concentration by gel-free semen volume, and no significant difference was observed (Figure D.3). Analysis of spermatozoal viability showed no significant differences between treatment groups (Figure D.4). No effects of treatment by time were observed on TMOT, PMOT, or DNA quality (Figures D.5, D.6, and D.7, respectively). Variations in fresh semen parameters of individual stallions within groups can be viewed in Figures E.1 to E.7.

Mean percentages of normal spermatozoa and morphological abnormalities are presented in Table 12. Percent normal spermatozoa differed consistently between groups for the duration of the study, with ex stallions having a greater percentage of normal spermatozoa than non-ex $(\mathrm{P}<0.02)$ (Figure D.8). No effect of treatment by week was observed. Comparisons of respective morphological abnormalities between groups can be viewed in Figures F.1 to F.10.

\subsection{Cooled Semen}

Cooled semen samples (T24 and T48) were analyzed for VIAB, TMOT, PMOT, and COMPat. No significant differences were reported for any of the endpoints measured (Table 11). 
TABLE 12. Mean $( \pm$ SD) percentages of normal spermatozoa and morphologically abnormal spermatozoa observed in non-ex (control) and ex (treatment) stallions at 4 daily spermatozoa output determinations (Week 0, 4, 8, and 12) (n=4/group).

\begin{tabular}{|c|c|c|c|c|c|c|c|c|}
\hline & \multicolumn{2}{|c|}{ WEEK O } & \multicolumn{2}{|c|}{ WEEK 4} & \multicolumn{2}{|c|}{ WEEK 4} & \multicolumn{2}{|c|}{ WEEK 8} \\
\hline & NON-EX & EX & NON-EX & EX & NON-EX & EX & NON-EX & EX \\
\hline $\begin{array}{l}\text { NORMAL } \\
\text { CELL }\end{array}$ & $60.88 \pm 17.43$ & $7.25 \pm 5.92$ & $58.63 \pm 15.99$ & $74.25 \pm 3.97$ & $51.75 \pm 7.27$ & $73.25 \pm 6.13$ & $5.75 \pm 4.65$ & $71.25 \pm 6.50$ \\
\hline $\begin{array}{l}\text { ABNORMAL } \\
\text { HEAD }\end{array}$ & $14.63 \pm 9.69$ & $7.63 \pm 4.13$ & $11.75 \pm 0.65$ & $8.25 \pm 0.96$ & $12.25 \pm 2.63$ & $7.25 \pm 4.27$ & $13.75 \pm 8.42$ & $10.50 \pm 6.40$ \\
\hline $\begin{array}{l}\text { ABNORMAL } \\
\text { ACROSOME }\end{array}$ & $1.50 \pm 1.29$ & $0.25 \pm 0.50$ & $3.88 \pm 3.33$ & $0.88 \pm 0.63$ & $4.50 \pm 4.65$ & $1.25 \pm 1.26$ & $4.75 \pm 2.06$ & $1.75 \pm 1.71$ \\
\hline $\begin{array}{l}\text { DETACHED } \\
\text { HEAD }\end{array}$ & $2.25 \pm 1.71$ & $3.00 \pm 3.67$ & $3.00 \pm 3.46$ & $1.38 \pm 1.25$ & $5.25 \pm 3.30$ & $2.25 \pm 1.89$ & $4.50 \pm 2.65$ & $3.25 \pm 2.50$ \\
\hline $\begin{array}{l}\text { PROXIMAL } \\
\text { DROPLET }\end{array}$ & $12.50 \pm 9.60$ & $10.75 \pm 2.75$ & $11.38 \pm 7.02$ & $7.88 \pm 4.13$ & $10.75 \pm 2.87$ & $9.00 \pm 5.60$ & $9.75 \pm 1.50$ & $6.25 \pm 1.71$ \\
\hline $\begin{array}{l}\text { DISTAL } \\
\text { DROPLET }\end{array}$ & $6.358 \pm 3.28$ & $4.88 \pm 4.25$ & $4.00 \pm 2.80$ & $4.88 \pm 3.40$ & $5.25 \pm 3.30$ & $5.75 \pm 5.91$ & $6.00 \pm 7.35$ & $3.25 \pm 1.26$ \\
\hline $\begin{array}{l}\text { ABNORMAL } \\
\text { MIDPIECE }\end{array}$ & $6.13 \pm 3.68$ & $3.25 \pm 1.32$ & $7.38 \pm 4.99$ & $4.88 \pm 1.93$ & $16.00 \pm 4.32$ & $4.50 \pm 2.08$ & $11.75 \pm 3.77$ & $7.75 \pm 6.24$ \\
\hline $\begin{array}{l}\text { BENT } \\
\text { MIDPIECE }\end{array}$ & $1.25 \pm 0.29$ & $0.63 \pm 0.75$ & $4.00 \pm 3.83$ & $1.63 \pm 1.31$ & $3.50 \pm 1.91$ & $1.25 \pm 0.50$ & $3.50 \pm 2.38$ & $1.50 \pm 0.58$ \\
\hline $\begin{array}{l}\text { BENT } \\
\text { TAIL }\end{array}$ & $1.00 \pm 0.71$ & $0.75 \pm 0.29$ & $2.00 \pm 1.58$ & $1.38 \pm 0.48$ & $2.50 \pm 1.29$ & $0.75 \pm 1.50$ & $2.25 \pm 0.96$ & $2.25 \pm 1.26$ \\
\hline $\begin{array}{l}\text { COILED } \\
\text { TAIL }\end{array}$ & $1.88 \pm 1.44$ & $0.88 \pm 0.48$ & $3.38 \pm 5.76$ & $0.75 \pm 0.50$ & $3.25 \pm 0.96$ & $1.00 \pm 0.00$ & $2.50 \pm 1.00$ & $0.75 \pm 0.50$ \\
\hline $\begin{array}{l}\text { PREMATURE } \\
\text { GERM CELL }\end{array}$ & $0.13 \pm 0.25$ & $0.38 \pm 0.48$ & $0.13 \pm 0.25$ & $0.13 \pm 0.25$ & $0.25 \pm 0.50$ & $0.00 \pm 0.00$ & $0.25 \pm 0.50$ & $0.00 \pm 0.00$ \\
\hline
\end{tabular}

*No significant differences in normal cells were observed $(\mathrm{P}>0.05)$; statistical analyses were not performed on abnormalities 


\subsection{Evaluation of Fitness Level}

Mean ( \pm SD) HR, RR, and blood lactate concentrations were analyzed to determine whether ex stallions achieved a higher level of fitness as a result of exercise protocol (Table 13). Although decreases in all endpoints (HR, RR, and lactate) at 28 min of SET were observed in both groups from pre-EP to post-EP, none were significant (Figures G.1 to G.3). However, differences in RR were reported at the 28 min time point of SET from pre-EP to Post EP $(\mathrm{P}<0.0002)$.

TABLE 13. Mean $( \pm$ SD) of heart rate $(H R)$ and respiration rate $(R R)$ of non-ex (control) and ex (treatment) stallions, recorded at 28 min of standard exercise tests conducted before exercise period (pre-EP) and upon completion of the exercise period (post-EP) (n=4/group).

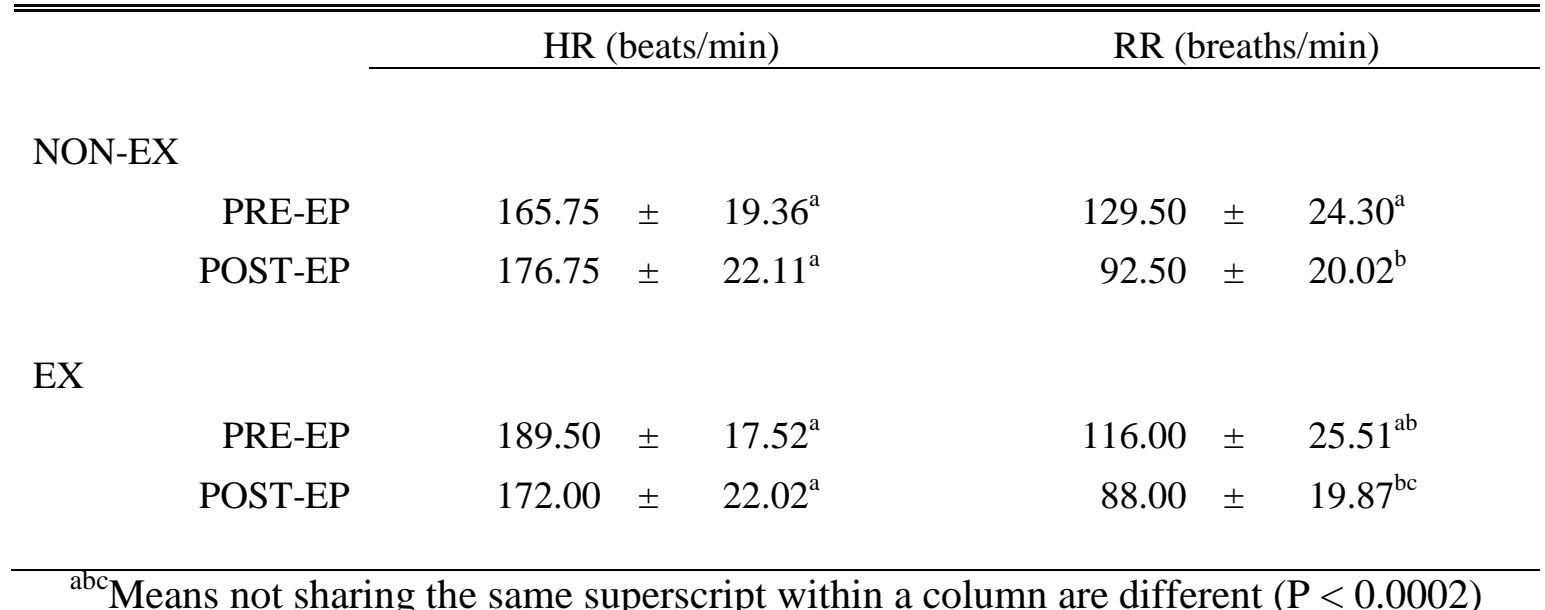

${ }^{\mathrm{abc}}$ Means not sharing the same superscript within a column are different $(\mathrm{P}<0.0002)$ 


\section{DISCUSSION}

To our knowledge, no research has attempted to implant a thermal sensory device into the scrota of stallions. Therefore, the first and foremost objective of the current study sought to determine whether subdermal thermal sensors could be applied to the measurement of neck and scrotal temperatures. No complications arose during scrotal implantation surgery, and one day after the surgery, no swelling, edema, increase in temperature, or trauma to the testicle was apparent. Similarly, no adverse reactions were recorded for the implantation of devices into the subdermis of the neck. One stallion did lose his scrotal implant within $1 \mathrm{wk}$ of surgery; however, it was replaced with no complications. Subdermal thermal sensors can be applied and maintained to the measurement of neck and scrotal temperatures for 3 to 4 mo.

The second objective of the current study was to determine the effect of exercise on RCT, SQST, and SQNT of stallions in hot and humid conditions. Horses exercising in such conditions are not extremely effective at thermoregulation, as they use twice the muscle mass as do humans for locomotion, yet they only have about 50\% the skin surface area per unit body mass for evaporative heat loss (Lindinger, 1999). Additionally, since humid conditions cause a decrease in the water vapor gradient for evaporation of sweat, the rate of evaporative heat loss is reduced (Lindinger, 1999). Peak mean AMBT and corresponding HUM during exercise protocol of the current study ranged between 44.0 to $44.7^{\circ} \mathrm{C}$ and 46.7 to $47.6 \%$, respectively. Ambient temperature and HUM were used to calculate THI, which can be defined as the apparent 
temperature in the shade (NOAA, 2010). Peak mean THI during exercise protocol of this project was calculated using AMBT measurements in the heat of the sun, resulting in an extremely high range of 65.7 to $66.5^{\circ} \mathrm{C}$, considered extremely dangerous to humans (NOAA, 2010). If humans have a higher capacity for evaporative cooling than do horses, then extreme THI to which stallions were subjected during this study could be potentially more dangerous for horses than for humans. Ambient temperature, HUM, and THI to which stallions were subjected during exercise protocol of the current study were extremely hot and humid, as is characteristic for south-central Texas. Stallions being intensely exercised in such climates may experience difficulty dissipating body heat, resulting in inefficient thermoregulation of the body and, subsequently, the scrotum.

However, stallions used in the current study did not appear to have difficulty dissipating heat generated from the exercise protocol. Increases in RCT of stallions in the present study were not as high as increases in core temperature previously documented in exercised horses (Webb et al., 1987; Scott, 1992; Kohn et al., 1999). Quarter horses conditioned aerobically and trained for cutting work in 45-min bouts experienced a mean RCT of approximately $38.9^{\circ} \mathrm{C}$ during exercise, with a higher mean temperature of $40.5^{\circ} \mathrm{C}$ recorded at 30 min recovery (Webb et al., 1987). Daily aerobic conditioning by galloping around an oval track for approximately $50 \mathrm{~d}$ prior to a final anaerobic exercise test consisting of four 600-m sprints resulted in maximal core body temperatures averaging $41^{\circ} \mathrm{C}$ at 15 min recovery (Scott, 1992). Thoroughbred horses were also subjected to 46-min standard exercise tests in varying AMBT and HUM on a 
high speed treadmill, with RCT reaching $41.5^{\circ} \mathrm{C}$ in hot and humid conditions (Kohn et al., 1999). The core temperatures achieved during the exercise protocols employed in these projects were approaching $42^{\circ} \mathrm{C}$, which is considered the critical body temperature in the equine that may elicit voluntary exhaustion (Lindinger, 1999). Although mean peak RCT of $39.2^{\circ} \mathrm{C}$ and $\mathrm{SQNT}$ of $39.9^{\circ} \mathrm{C}$ were achieved, these increases in temperature were not extreme. Therefore, exercise protocol used on the current study cannot be considered as an intense regimen capable of producing maximal core body temperatures. Also, the current study cannot define the period of recovery, as the earliest recovery temperatures were recorded at 60 min-post exercise when temperatures had returned to resting values. By recording stallion temperatures every 10 -min for a period of 60 -min post exercise, a more defined recovery period would have been apparent.

All stallions experienced an increase in RCT, SQNT, and SQST at 22 and $30 \mathrm{~min}$ of exercise protocol, which was likely due to the extreme THI and related AMBT and HUM during those time points. All stallions were removed from their shaded stalls at 0 min and were either tied (non-ex) or exercised (ex) in the heat of the sun; this explains the temperature increases seen in the control (non-ex) group. All stallions were returned to their shaded stalls immediately following the 30 min time point, which elicited an apparent decrease in RCT, SQNT, and SQST at 60 min-post and 120 min-post exercise in all stallions.

The third and final objective of the present study sought to determine the relationship between changes in RCT, SQST, and SQNT and sperm quality. Abundant literature describes changes in concentration, number of spermatozoa per ejaculate, 
motility, morphology, and integrity of spermatozoal chromatin structure resulting from SST increases of 1 to $3.5^{\circ} \mathrm{C}$ via scrotal insulation for prolonged periods of time (Freidman et al., 1991; Blanchard et al., 2000; Love and Kenney, 1999; Ross and Entwistle, 1979; Vogler et al., 1993; McNitt and First, 1970; Barth and Bowman, 1994). In the present study, a mean increase in SQST of only $0.8^{\circ} \mathrm{C}$ was achieved in ex stallions from 0 to 22 min of exercise. No adverse effects on semen parameters were observed, which is similar to results from studies in men that reported no changes in semen quality due to SQST increases of 0.8 to $1.0^{\circ} \mathrm{C}$ (Wang et al., 1997). Rams subjected to a 1.4 to $2.2^{\circ} \mathrm{C}$ increase in SQST for $21 \mathrm{~d}$ experienced a significant decrease in fertility (Mieusset et al., 1992). Clearly, prolonged increases in scrotal temperature due to insulation have detrimental effects on reproductive parameters, as the scrotum cannot regulate and dissipate heat. Stallions in the current study did experience elevated RCT and SQNT during exercise; however, the increase only lasted a maximum of $30 \mathrm{~min} / \mathrm{d}$ for $4 \mathrm{~d} / \mathrm{wk}$, and SQST never increased significantly. The implemented exercise protocol more appropriately mirrored current industry standard exercise regimens for performance horses, and only intermittent increases in temperature due to exercise were noted. Since Miniature Horse stallions were used, effects of breed differences may exist. Nevertheless, results of the current study suggest that daily short-term exercise in hot and humid climates has little effect on RCT and SQST and no adverse effects on semen quality.

Most studies have used SST as the endpoint to determine thermoregulatory efficiency of the testes. However, studies utilizing intratesticular thermistors measured 
the temperature of the testicular parenchyma and found a low correlation between SST and ITT (Kastelic et al., 1995). Subcutaneous scrotal temperature displayed a low to moderate correlation with ITT (Kastelic et al., 1995), which suggests that SQST may be a slightly more accurate measurement than SST for predicting ITT. Subcutaneous scrotal temperature in the current study showed a correlation with RCT $\left(r_{s}=0.761\right)$, $\operatorname{AMBT}\left(r_{\mathrm{s}}=0.625\right)$ and THI $\left(r_{\mathrm{s}}=0.629\right)$, which implies that further studies might be successful in formulating an equation to determine SQST based on RCT.

Standard exercise tests to which the stallions were subjected during the current study displayed no significant evidence that a higher level of fitness was achieved by the exercise protocol employed. Although respiration rates of ex stallions were significantly lower during the post-EP SET, heart rates did not differ from the pre- to post-EP SET. Blood lactate levels may have indicated a higher level of fitness post-EP; however, some samples were hemolyzed, which compromised the integrity of the assay. Therefore, the exercise protocol can only be considered as minimally intense, as it did not achieve greater fitness in the stallions subjected to it.

Results of the current study are consistent with reports by Dinger et al. (1986) that showed no change in semen parameters of stallions being subjected to minimal or short term daily exercise. A conflicting report claimed that stallions trained for intermediate and advanced levels of dressage or show jumping for 1 to $2 \mathrm{~h} / \mathrm{d}$ had significantly higher spermatozoal motility than stallions used for breeding alone (Lange et al., 1997). However, these stallions were not blocked into groups according to age or reproductive parameters, and no specific exercise protocol was defined. Further 
contradictory literature reported a decrease in the percent normal cells and motility of fresh and frozen-thawed semen during and up to 1 month after intense exercise until exhaustion for $2 \mathrm{~d} / \mathrm{wk}$ (Janett et al., 2006). Neither rectal nor scrotal temperatures were measured during this study; therefore, it cannot be determined whether the detriment to spermatozoal parameters was due to increased temperatures or stress levels.

Future research on this topic may benefit from a longer exercise protocol. In the current study, the stallion scrotum appeared to efficiently dissipate heat during and after exercise; however, prolonged exercise protocol would likely result in prolonged elevated body temperatures, which might have more of an effect on reproductive parameters. Staempfli et al. (2006) recorded peak SST values when horses were at rest following exercise; the faster the horse moved, the more efficient the scrotal cooling. Roping and cutting stallions are subjected to intense but interrupted exercise regimens, stopping between performances. These horses likely experience elevated temperatures during exercise, followed by a period of standing at rest, followed by exercise. During rest periods, the scrotum may not receive optimal air flow; therefore, efficient scrotal cooling could be hindered. A future study could examine the effects of interrupted exercise on stallion reproductive efficiency. 


\section{CONCLUSION}

The objectives of the current study were to: determine whether subdermal thermal sensors could be applied to the measurement of SQNT, RCT, and SQST; determine the effect of exercise on RCT, SQST, and SQNT of stallions in hot and humid environments; determine the relationship between changes in RCT, SQST, and SQNT and sperm quality. The results indicate that SQST was not affected by exercise protocol, and thus semen parameters remained unaffected.

Although an increase in core body temperature was successfully induced by exercise protocol, scrotal temperatures were not significantly affected, indicating efficient thermoregulation of the testes by the scrotum. Since the testes experienced no significant thermal insult during the exercise protocol, no significant changes in fresh or cooled semen parameters were evident as a result of exercise or elevated core temperature.

No fever, edema, or other deleterious effects were noted from the subdermal implantation of thermal sensory devices into the necks or scrotums of the stallions in this study. Therefore, the implantation of thermal sensory devices into the subdermis of the scrotum can be considered as a safe and effective method to monitor testicular thermal stress. 


\section{LITERATURE CITED}

Barth, A.D. and P.A. Bowman. 1994. The sequential appearance of spermatozoa abnormalities after scrotal insulation or dexamethasone treatment in bulls. Can. Vet. J. 35:93-102.

Blanchard, T., D. Varner, L. Johnson, J. Roser, J. Hill, and C. Miller. 2000. Testicular and hormonal changes in stallions with thermally induced testicular degeneration. J. Reprod. Fertil. Suppl. 56:51-59.

Bonde, J.P. 1992. Semen quality in welders exposed to radiant heat. Br. J. Ind. Med. 49:5-10.

Casady, R.B., R.M. Myers, and J.E. Legates. 1953. The effect of exposure to high ambient temperature on spermatogenesis in the dairy bull. J. Dairy Sci. 36:1423.

Coulter, G.H., P.L. Senger, and D. Bailey. 1988. Relationship of scrotal surface temperature measured by infrared thermography to subcutaneous and deep testicular temperature in the ram. J. Reprod. Fertil. 84:417-423.

Davies Morel, M.C.G., and V. Gunnarsson. 2000. A survey of the fertility of Icelandic stallions. Anim. Reprod. Sci. 64:49-64.

Davies Morel, M.C.G. 2008. Equine Reproductive Physiology, Breeding, and Stud Management. CAB International, Wallingford, Oxfordshire, UK.

Deloitte. 2005. The Economic Impact of the Horse Industry in the United States. American Horse Council Federation. Washington, DC.

Dinger J. E., E. E. Noiles, T. A. Hoagland. 1986. Effect of controlled exercise on semen characteristics in two-year-old stallions. Therio. 25:525-535.

Freidman, R., M. Scott, S.E. Heath, J.P. Hughes, P.F. Daels, and T.Q. Tran. 1991. The effects of increased testicular temperature on spermatogenesis in the stallion. J. Reprod. Fertil., Suppl. 44:127-134.

Gebauer M.R, B.W. Pickett, J.L. Voss, and E.E. Swierstra. 1974. Reproductive physiology of the stallion: Daily spermatozoa output and testicular measurements. J. Am. Vet. Med. Assoc. 165:711-713. 
Gebreegziabher, Y., E. Marcos, W. McKinnon, and G. Rogers. 2004. Spermatozoa characteristics of endurance trained cyclists. Int. J. Sports Med. 25(4):247-251.

Henneke, D. R., G. D. Potter, J. L. Kreider, and B. F. Yeates. 1983. Relationship between condition score, physical measurements and body fat percentage in mares. Equine Vet. J. 15(4):371-372.

Janett, F., C. Burkhardt, D. Burger, I. Imboden, M. Hassig, and R. Thun. 2006. Influence of repeated treadmill exercise on quality and freezability of stallion semen. Therio. 65:1737-1749.

Jasko D.J., T.V. Little, D.H. Lein, and R.H. Foote. 1992. Comparison of spermatozoal movement and semen characteristics with fertility in stallions: 64 cases (19871988). J. Am. Vet. Med. Assoc. 200:979-985.

Jones, L.L., D.D. Householder, P.G. Gibbs, and G.D. Potter. 1993. Population Estimates for the Texas Horse Industry. Departmental Information Report No. 94-2. Department of Agricultural Economics, Texas A\&M University, College Station.

Jung, A. and H.C. Schuppe. 2007. Influence of genital heat stress on semen quality in humans. Int. J. Androl. 39:203-215.

Kastelic, J.P., G.H. Coulter, and R.B. Cook. 1995. Scrotal surface, subcutaneous, intratesticular, and intraepididymal temperatures in bulls. Therio. 44:147-152.

Kenney R.M., J.P. Hurtgen, and R. Pierson. 1983. Theriogenology and the equine, part II, the stallion. Therio. 9:88-90.

Kohn, C.W., K.W. Hinchcliff, and K.H. McKeever. 1999. Effect of ambient temperature and humidity on pulmonary artery temperature of exercising horses. Eq. Vet. J., 31:404-411.

Konavongkrit, A., A. Suriyasomboon, N. Lundeheim, T. Heard, and S. Einarsson. 2005. Management and spermatozoa production of boars under differing environmental conditions. Therio. 63:657-667.

Lange, J., S. Matheja, E. Klug, C. Aurich, and J.E. Aurich. 1997. Influence of training and competition on the endocrine regulation of testicular function and on semen parameters in stallions. Reprod. Dom. Anim. 32:297-302.

Lindinger, M.I. 1999. Exercise in the heat: Thermoregulatory limitations to performance in humans and horses. Can. J. Appl. Physiol. 24(2):152-163. 
Love, C.C., M.C. Garcia, F.R. Riera, and R.M. Kenney. 1991. Evaluation of measures taken by ultrasonography and caliper to estimate testicular volume and predict daily spermatozoa output in the stallion. J. Reprod. Fertil. Suppl. 44:99-105.

Love, C.C. and R.M. Kenney. 1998. The relationship of increased susceptibility of spermatozoa DNA to denaturation and fertility in the stallion. Therio. 52:955972.

Love, C.C. and R.M. Kenney. 1999. Scrotal heat stress induces altered spermatozoa chromatin structure associated with a decrease in protamine disulfide bonding in the stallion. Biol. Reprod. 60:615-620.

Marai, I.F.M, A.A.M. Habeeb, and A.E. Gad. 2002. Reproductive traits of male rabbits as affected by climatic conditions in the subtropical environment of Egypt. J. Anim. Sci. 75:451-458.

McKinnon, A.O. and J.L. Voss. 1993. Equine Reproduction. Lea \& Febiger, Malvern, PA.

McNitt, J.I. and N.L. First. 1970. Effects of 72-hour heat stress on semen quality in boars. Int. J. Biometeor. 14:373-380.

Mieusset, R., P. Quintana Casares, L.G. Sanchez Partida, S.F. Sowerbutts, J.L. Zupp, and B.P. Setchell. 1992. Effects of heating the testes and epididymides of rams by scrotal insulation on fertility and embryonic mortality in ewes inseminated with frozen semen. J. Reprod. Fertil. 94:337-343.

Mieusset, R. and L. Bujan. 1994. The potential of mild testicular heating as a safe, effective and reversible contraceptive method for men. Int. J. Androl. 17:186191.

Momen, M.N., F.B. Ananian, and I.M Fahmy. 2010. Effect of high environmental temperature on semen parameters among fertile men. Fertil. and Steril. 93:18841886.

National Oceanic and Atmospheric Administration (NOAA). 2010. Hourly relative humidities recorded during the summer of 2010. Accessed Oct. 20, 2010. http://www.noaa.com/pastweather.html.

Oristalgo Turner, R.M. 2007. Pathogenesis, diagnosis, and management of testicular degeneration in stallions. Clin. Tech. Equine Pract. 6:278-284. 
Perez-Crespo, M., B. Pintado, and A. Gutierrez-Adan. 2008. Scrotal heat stress in spermatozoa viability, spermatozoa DNA integrity and the offspring sex ratio in mice. Mol. Reprod. Dev. 75:40-47.

Rockett, J.C., F.L. Mapp, J.B. Garges, J.C. Luft, C. Mori, and D.J. Dix. 2001. Effects of hyperthermia on spermatogenesis, apoptosis, gene expression, and fertility in adult male mice. Biol. Reprod. 65:229-239.

Ross, A.D. and K.W. Entwistle. 1979. The effect of scrotal insulation on spermatozoal morphology and the rates of spermatogenesis and epididymal passage of spermatozoa in the bull. Therio. 11(2):111-129.

Sailer, B.L., L.J. Sarkar, J.A. Bjordahl, L.K. Jost, and D.P. Evenson. 1997. Effect of heat stress on mouse testicular cells and spermatozoa chromatin structure. J. Androl. 18:294-301.

Scott, B.D. 1992. Efficacy of a fat-supplemented diet to maintain muscle glycogen stores and reduce thermal stress in exercising Thoroughbred horses. Ph.D. Dissertation, Texas A\&M University, College Station, TX.

Shilkina, L.A. 1976. Effect of hyperthermia on spermatogenesis in mice and the role of heat training in adaptation of the sex cells to high temperature. Bull. Experi. Biol. Med. 82:1860-1863.

Staempfli, S., F. Janett, D. Burger, H. Kundig, I. Imboden, M. Hassig, and R. Thun. 2006. Effect of exercise and suspensory on scrotal surface temperature in the stallion. Therio. 66:2120-2126.

Stone, B.A. 1982. Heat induced infertility of boars: The inter-relationship between depressed spermatozoa output and fertility and an estimation of the critical air temperature above which spermatozoa output is impaired. Anim. Reprod. Sci. 4:283-299.

Thompson J.A., D.D. Love, K.L Stich, S.P. Brinsko, T.L. Blanchard, and D.D Varner. 2004. A Bayesian approach to prediction of stallion daily spermatozoa output. Therio. 62:1607-1617.

Vaamonde, D., M.E. Da Silva, M.S. Pablador, and J.L. Lancho. 2006. Reproductive profile of physically active men after exhaustive endurance exercise. Int. J. Sports Med. 27(9):680-689.

Vogler, C.J., J.H. Bame, J.M. DeJarnette, M.L. McGilliard, and R.G. Saacke. 1993. Effects of elevated testicular temperature on morphology characteristics of ejaculated spermatozoa in the bovine. Therio. 40:1207-1219. 
Wang C., V. McDonald, A. Leung, L. Superlano, N. Berman, L Hull, and R.S. Swerdloff. 1997. Effect of increased scrotal temperature on spermatozoa production in normal men. Fertil. Steril. 68:334-339.

Watanabe, A. 1959. The effect of heat on the human spermatogenesis. Kyushu J. Med. Sci. 10:101-117.

Webb, S.P., G.D. Potter, J.W. Evans, C.A. Schwab, and B.D. Scott. 1987. Physiological responses of cutting horses to exercise testing and training. Pages 351-356 in Proceedings of the $10^{\text {th }}$ Equine Nutritional Physiology Symposium. Ft. Collins, CO.

Westervelt, R.G., J.R. Stouffer, H.F. Hintz, and H.F. Schryver. 1976. Estimating fatness in horses and ponies. J. Anim. Sci. 43:781-785.

Wettemann, R.P., M.E. Wells, I.T. Omtvedt, C.E. Pope, and E.J. Turman. 1976. Influence of elevated temperature on reproductive performance of boars. J. Anim. Sci. 42:664-669. 


\section{APPENDIX A}

\section{PROJECT ORGANIZATION}

TABLE A.1 Timeline of study protocol.

\begin{tabular}{|c|c|c|c|c|c|c|c|c|}
\hline $\begin{array}{c}\text { Dates } \\
(2010) \\
\end{array}$ & Week & Sun & Mon & Tues & Wed & Thurs & Fri & Sat \\
\hline $4 / 4-7 / 3$ & & & & INITIAL RES & PERIOD & & & \\
\hline $5 / 27 \& 6 / 3$ & & & & IMPLANT S & RGERIES & & & \\
\hline $7 / 4-7 / 10$ & 0 & BCS & DSO 1 & DSO 1 & DSO1 & DSO 1 & DSO 1 & EX \\
\hline $7 / 11-7 / 17$ & 1 & BCS & pre-EP SET & pre-EP SET & & & EX & EX \\
\hline $7 / 18-7 / 24$ & 2 & BCS & EX & EX & & & EX & EX \\
\hline $7 / 25-7 / 31$ & 3 & BCS & EX & EX & & & EX & EX \\
\hline $8 / 1-8 / 7$ & 4 & BCS & DSO 2 & DSO 2 & DSO 2 & DSO 2 & DSO 2 & EX \\
\hline $8 / 8-8 / 14$ & 5 & BCS & EX & EX & & & EX & EX \\
\hline $8 / 15-8 / 21$ & 6 & BCS & EX & EX & & & EX & EX \\
\hline $8 / 22-8 / 28$ & 7 & BCS & EX & EX & & & EX & EX \\
\hline $8 / 29-9 / 4$ & 8 & BCS & DSO 3 & DSO 3 & DSO 3 & DSO 3 & DSO 3 & EX \\
\hline $9 / 5-9 / 11$ & 9 & BCS & EX & EX & & & EX & EX \\
\hline $9 / 12-9 / 18$ & 10 & BCS & EX & EX & & & EX & EX \\
\hline $9 / 19-9 / 25$ & 11 & $\mathrm{BCS}$ & EX & EX & & & EX & EX \\
\hline $\begin{array}{l}9 / 26-10 / 2 \\
10 / 2-10 / 5\end{array}$ & $\begin{array}{l}12 \\
13\end{array}$ & $\begin{array}{l}\text { BCS } \\
\text { BCS }\end{array}$ & $\begin{array}{c}\text { DSO } 4 \\
\text { post-EP SET }\end{array}$ & $\begin{array}{c}\text { DSO } 4 \\
\text { post-EP SET }\end{array}$ & DSO 4 & DSO 4 & DSO 4 & EX \\
\hline
\end{tabular}

${ }^{\mathrm{a}} \mathrm{BCS}=$ measurement of weight, body condition, and rump fat

${ }^{\mathrm{b}}$ DSO1-DSO4=daily spermatozoa output determinations

${ }^{c} \mathrm{EX}=$ exercise

${ }^{\mathrm{d}}$ pre-EP SET $=$ standard exercise test performed prior to the exercise period

e post-EP SET=standard exercise test performed at the cessation of the exercise period 


\section{APPENDIX B}

\section{DIET}

TABLE B.1 Chemical composition of forage diet.

\begin{tabular}{lcc}
\hline \hline & \multicolumn{2}{c}{ AMOUNT } \\
\cline { 2 - 3 } INGREDIENT & $\%$ & $\mathrm{ppm}$ \\
\hline Crude protein & 12.5 & \\
Acid detergent fiber & 27.5 & \\
Calcium & 0.5 & \\
Phosphorus & 0.25 & \\
Potassium & 1.54 & \\
Magnesium & 0.18 & \\
Sodium & & 702 \\
Zinc & & 39 \\
Iron & & 34 \\
Copper & & 13 \\
Manganese & & 96 \\
\hline
\end{tabular}

TABLE B.2 Chemical composition of concentrate diet.

\begin{tabular}{lcc}
\hline \hline & \multicolumn{2}{c}{ AMOUNT } \\
\cline { 2 - 3 } INGREDIENT & $\%$ & $\mathrm{ppm}$ \\
\hline Crude protein & 16.1 & \\
Acid detergent fiber & 12.1 & \\
Calcium & 0.93 & \\
Phosphorus & 0.6 & \\
Potassium & 1.06 & \\
Magnesium & 0.27 & \\
Sodium & & 3418 \\
Zinc & & 98.5 \\
Iron & & 29.5 \\
Copper & & 36 \\
Manganese & & 193 \\
\hline
\end{tabular}




\section{APPENDIX C}

\section{TEMPERATURE CHANGES OF INDIVIDUAL STALLIONS WITHIN GROUPS}
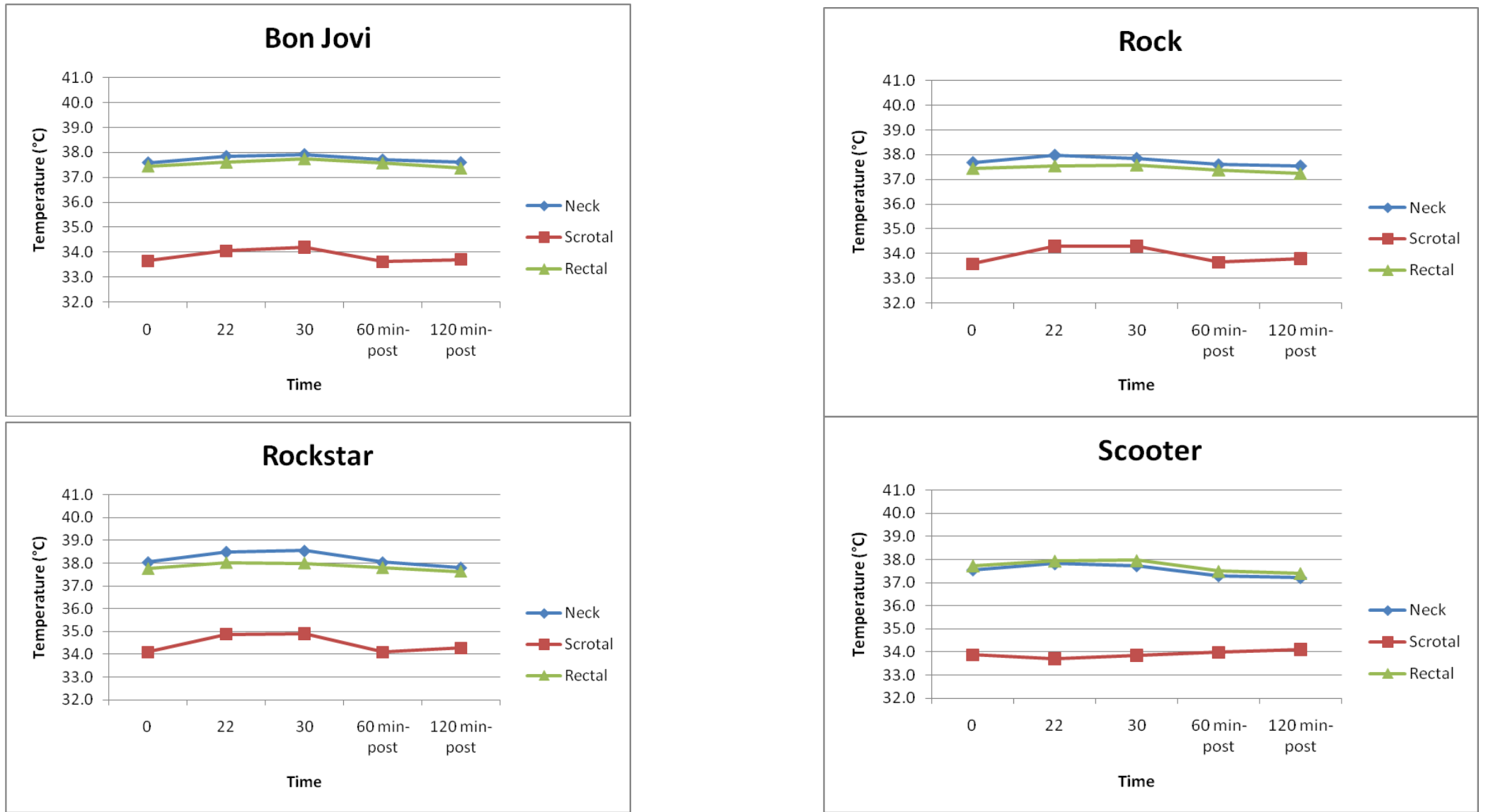

FIGURE C.1 Variations in mean temperature (neck, scrotal, and rectal) of individual stallions within the non-exercised (control) group $(n=4)$. 

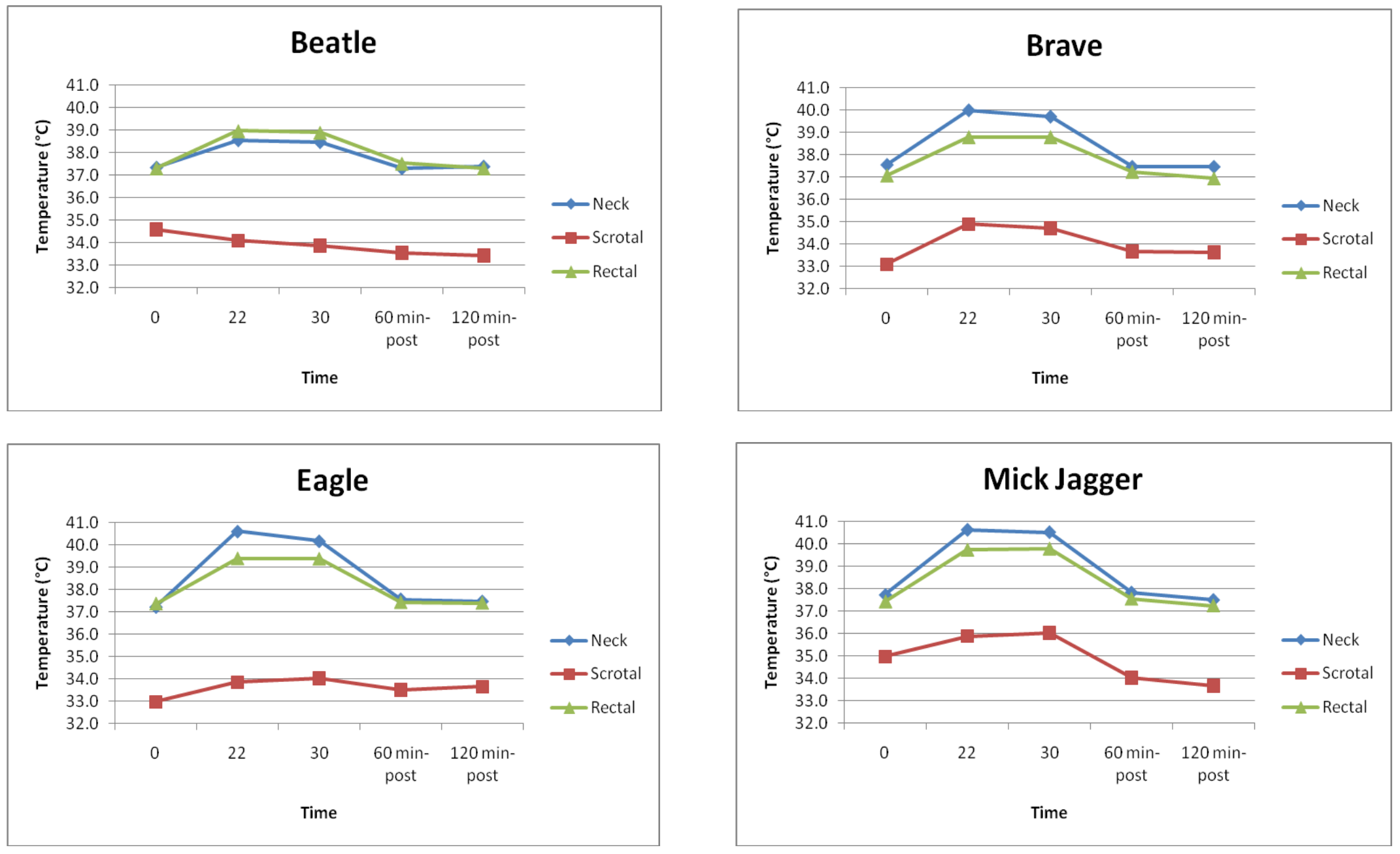

FIGURE C.2 Variations in mean temperature (neck, scrotal, and rectal) of individual stallions within the exercised (treatment) group $(n=4)$. 


\section{APPENDIX D}

FIGURES DISPLAYING MEAN SEMEN PARAMETERS WITHIN GROUPS

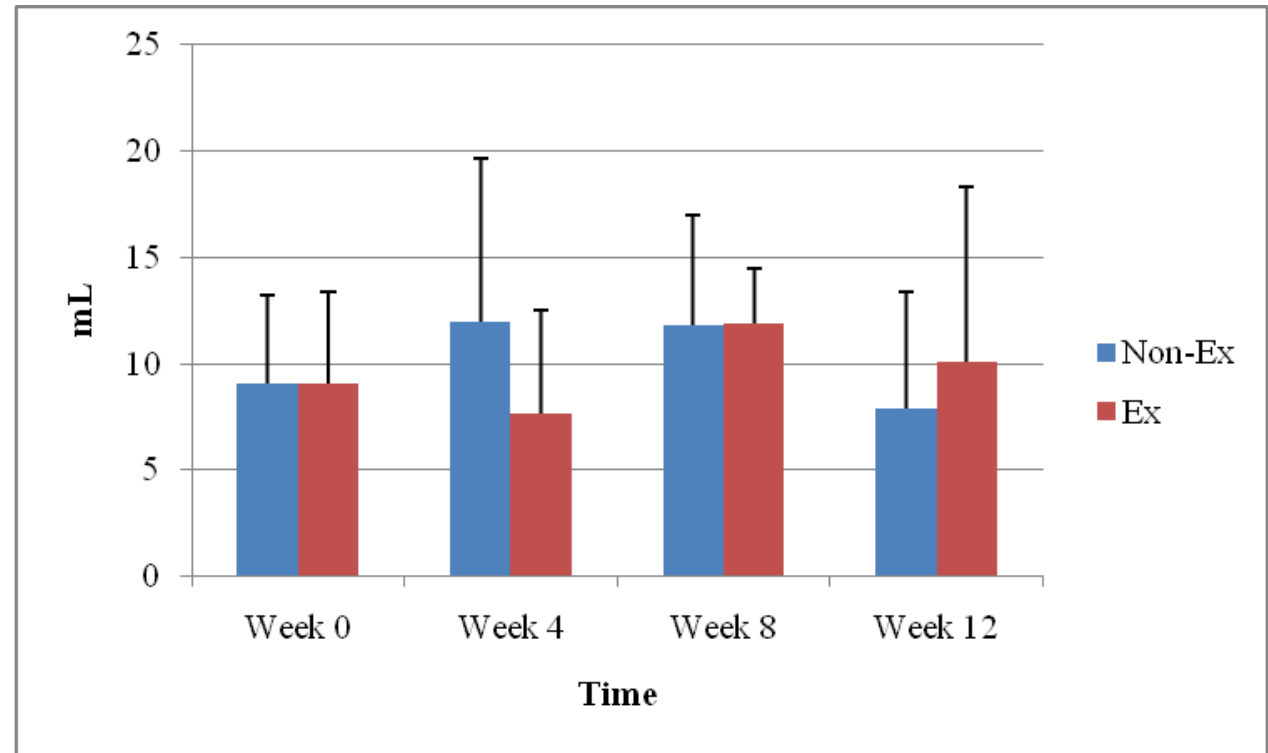

FIGURE D.1 Mean ( \pm SD) gel-free semen volume among non-ex (control) and ex (treatment) groups ( $\mathrm{n}=4 /$ group).

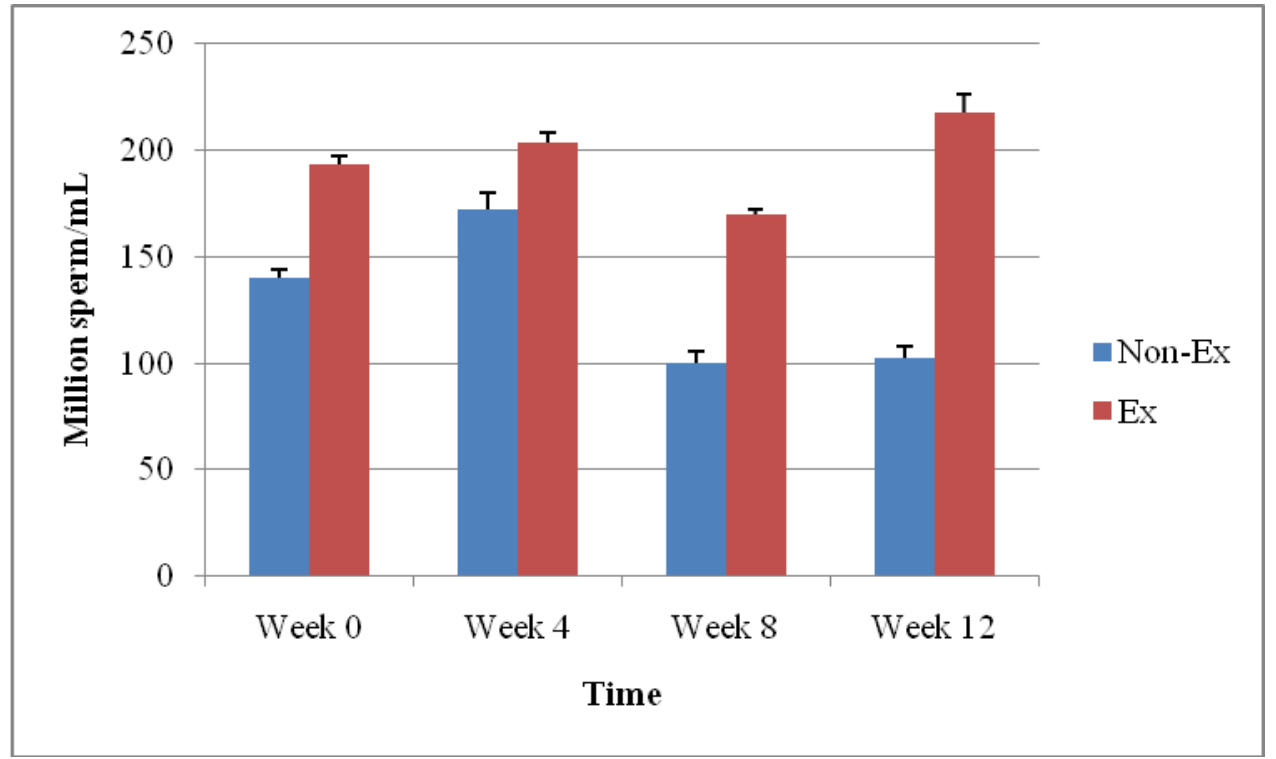

FIGURE D.2 Mean ( \pm SD) spermatozoal concentration among non-ex (control) and ex (treatment) groups ( $\mathrm{n}=4$ /group). 


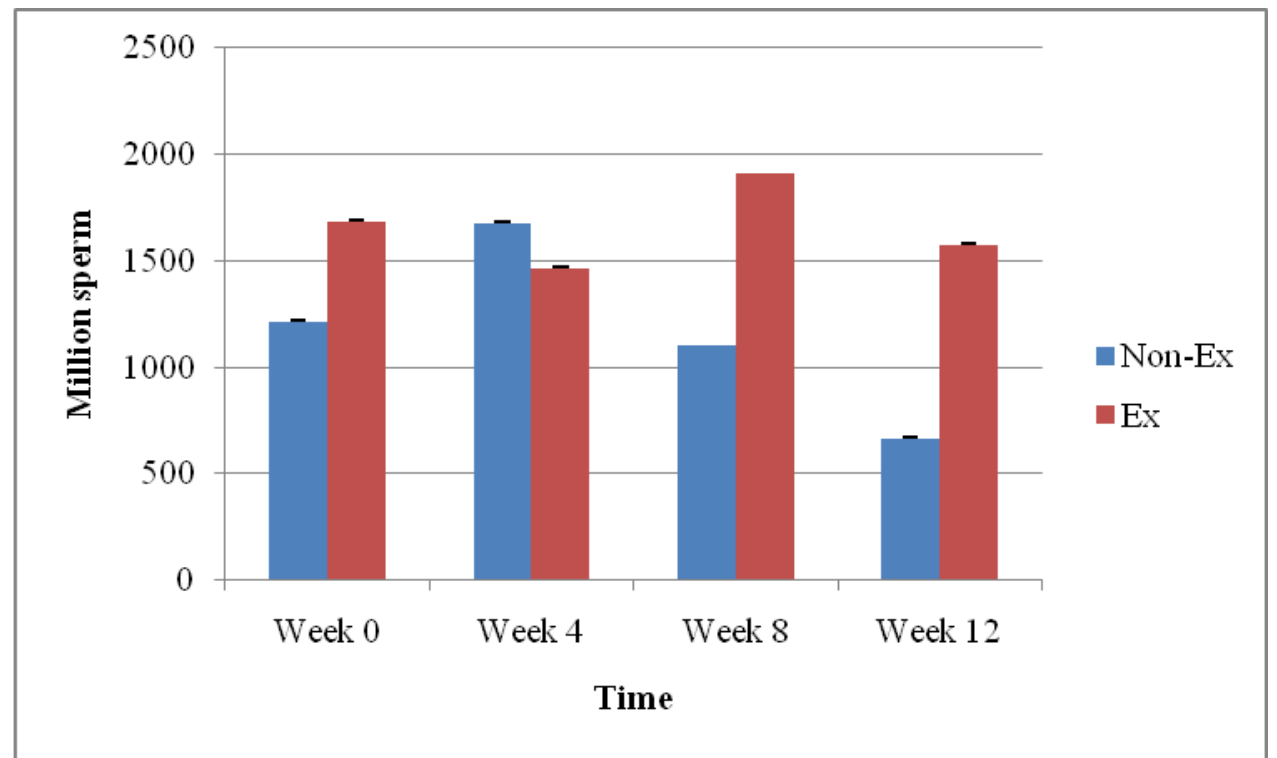

FIGURE D.3 Mean $( \pm$ SD) total number of cells among non-ex (control) and ex (treatment) groups ( $\mathrm{n}=4$ /group).

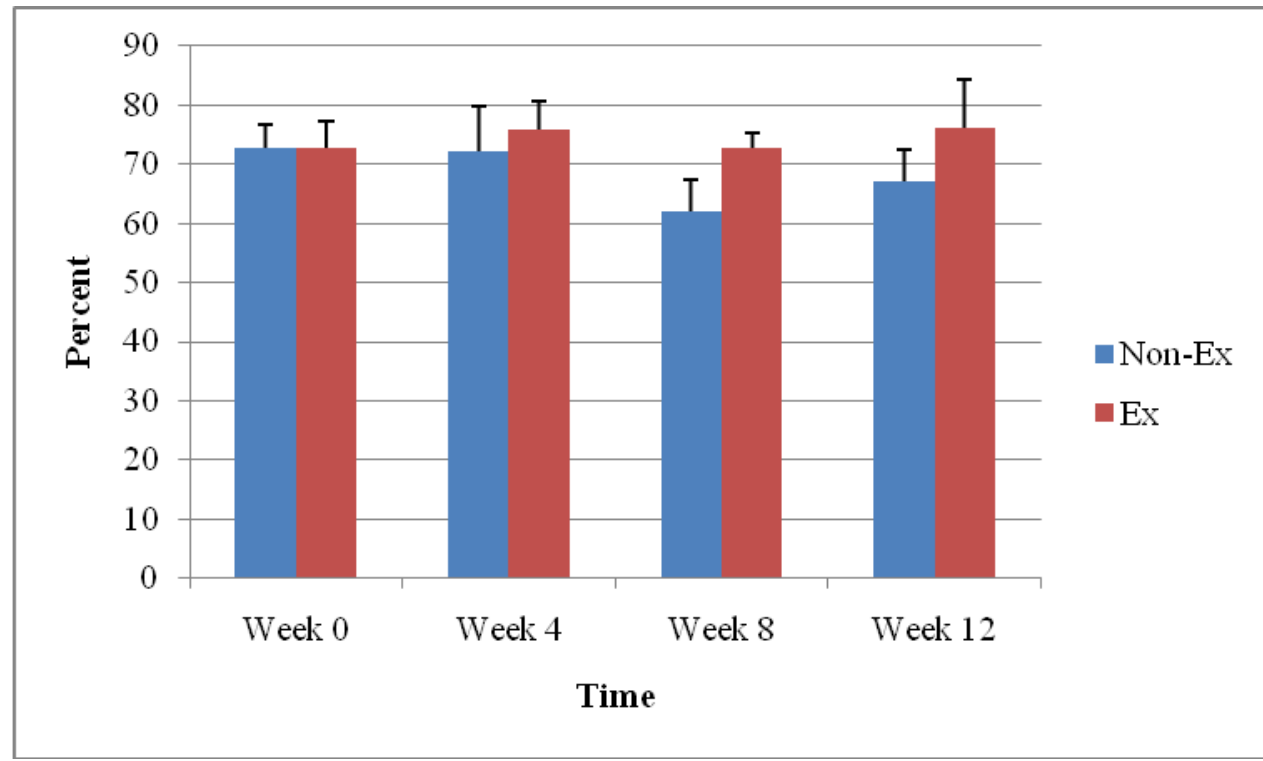

FIGURE D.4 Mean ( \pm SD) viability of spermatozoa among non-ex (control) and ex (treatment) groups ( $\mathrm{n}=4 /$ group). 


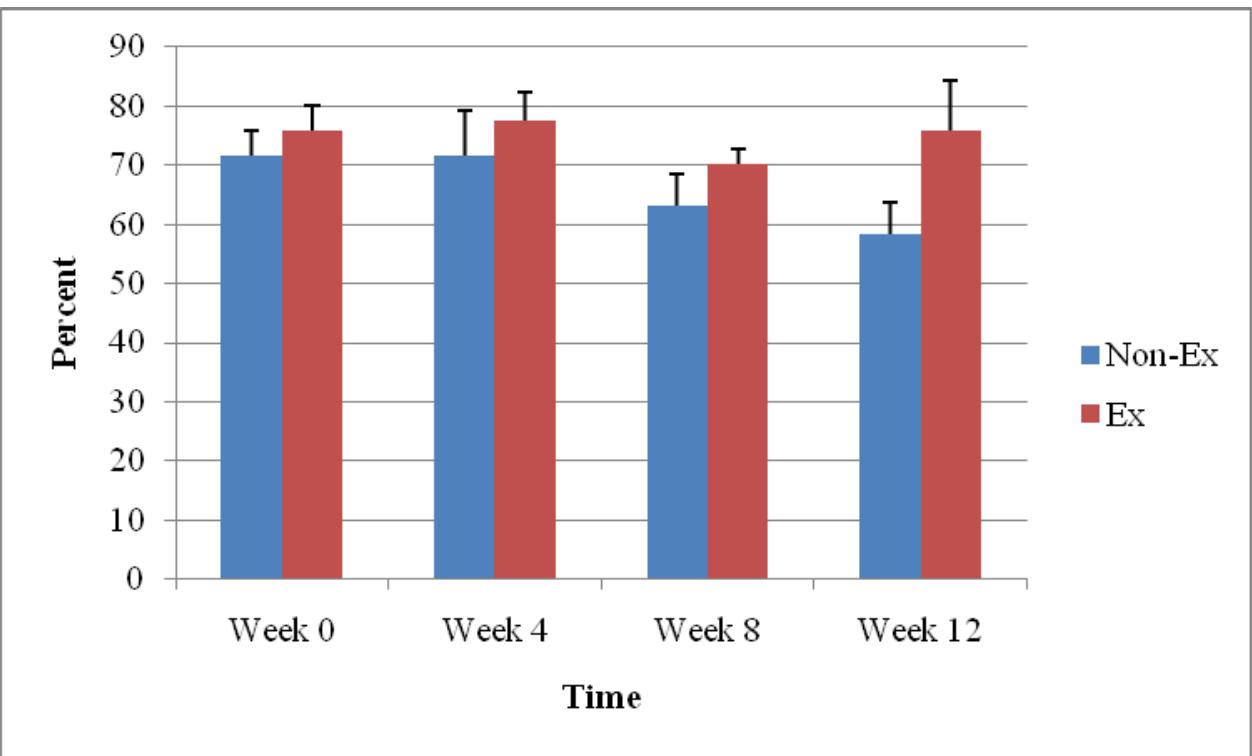

FIGURE D.5 Mean ( \pm SD) total motility of spermatozoa among non-ex (control) and ex (treatment) groups ( $\mathrm{n}=4$ /group).

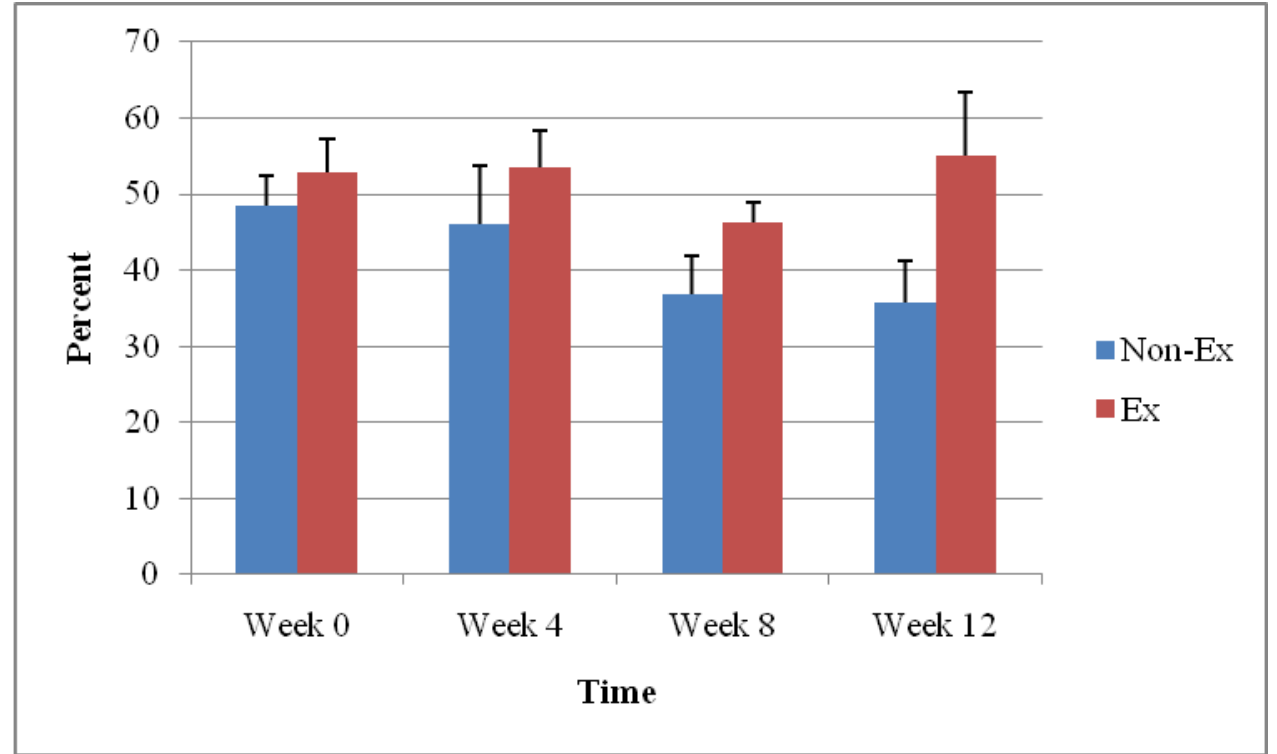

FIGURE D.6 Mean ( \pm SD) progressive motility of spermatozoa among non-ex (control) and ex (treatment) groups ( $\mathrm{n}=4$ /group). 


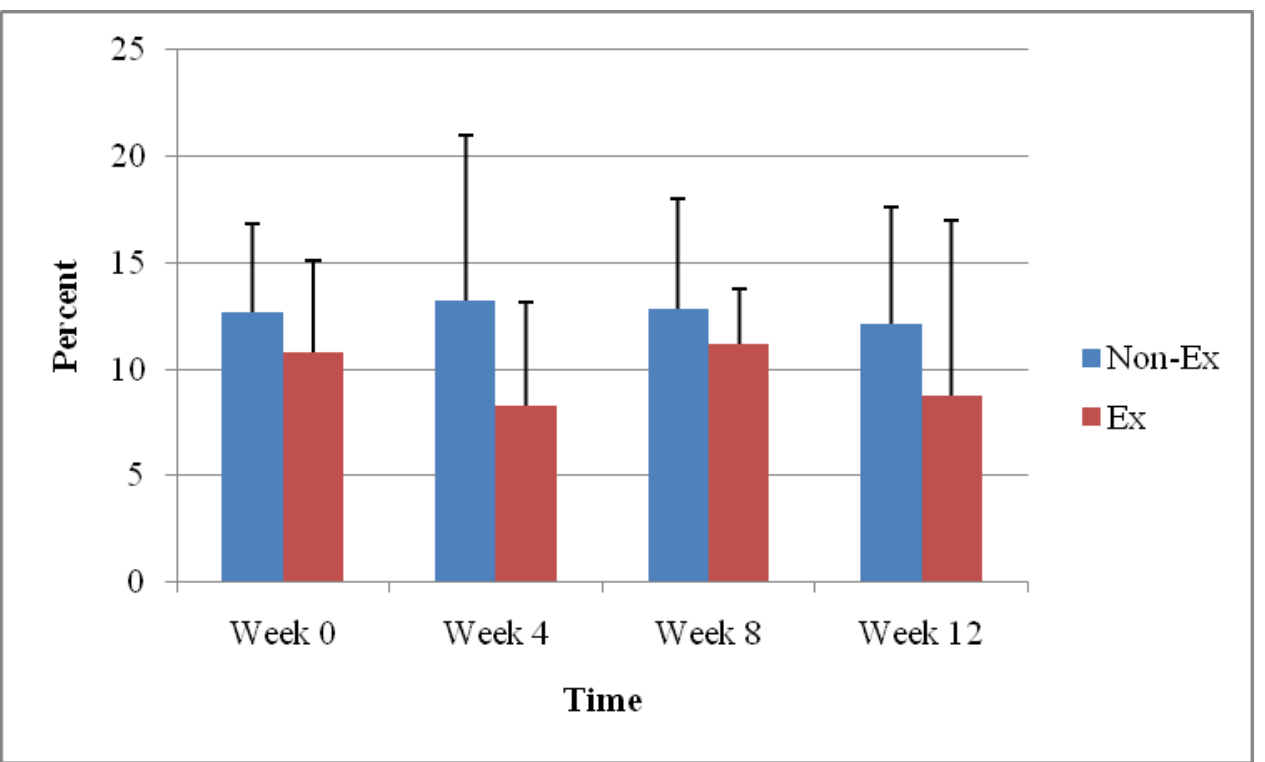

FIGURE D.7 Mean ( \pm SD) COMPat of Sperm Chromatin Structure Assay among nonex (control) and ex (treatment) groups (n=4/group).

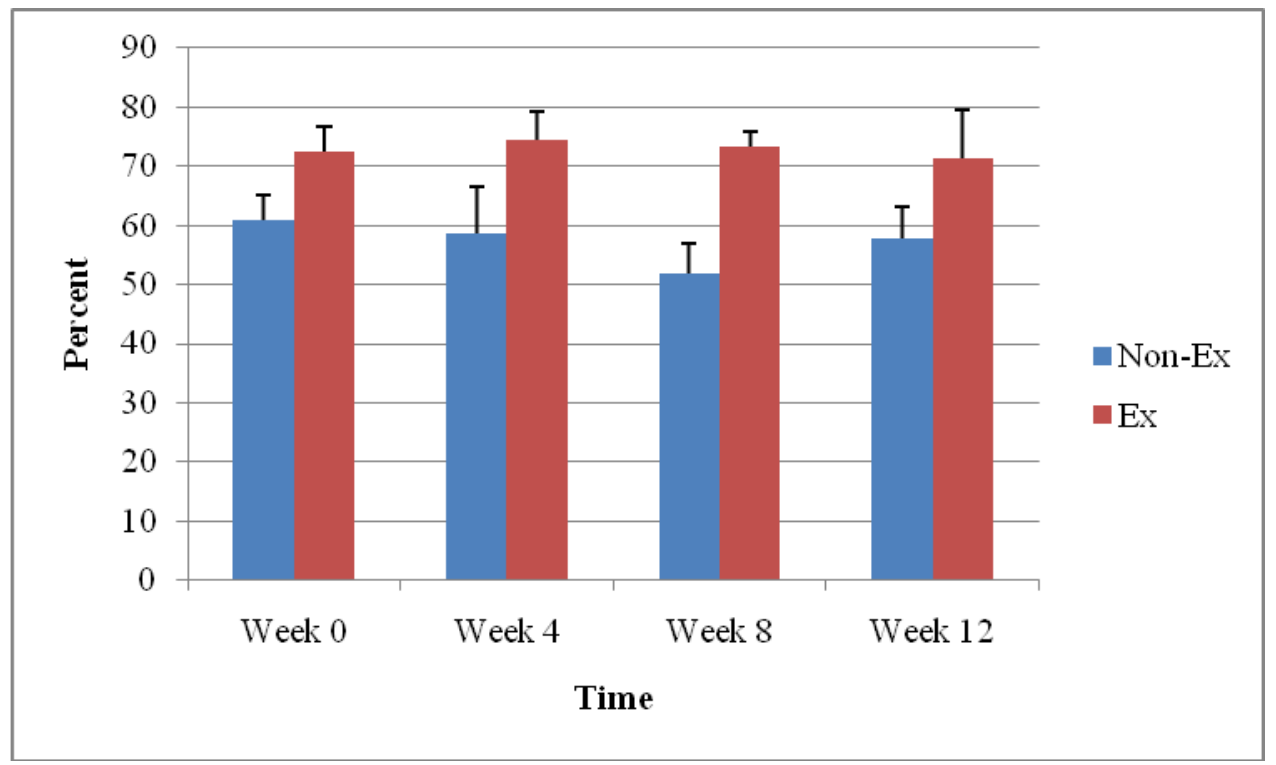

FIGURE D.8 Mean $( \pm$ SD) percentages of normal spermatozoa among non-ex (control) and ex (treatment) groups ( $\mathrm{n}=4$ /group). 


\section{APPENDIX E}

\section{INDIVIDUAL STALLION SEMEN PARAMETER FIGURES}

a)

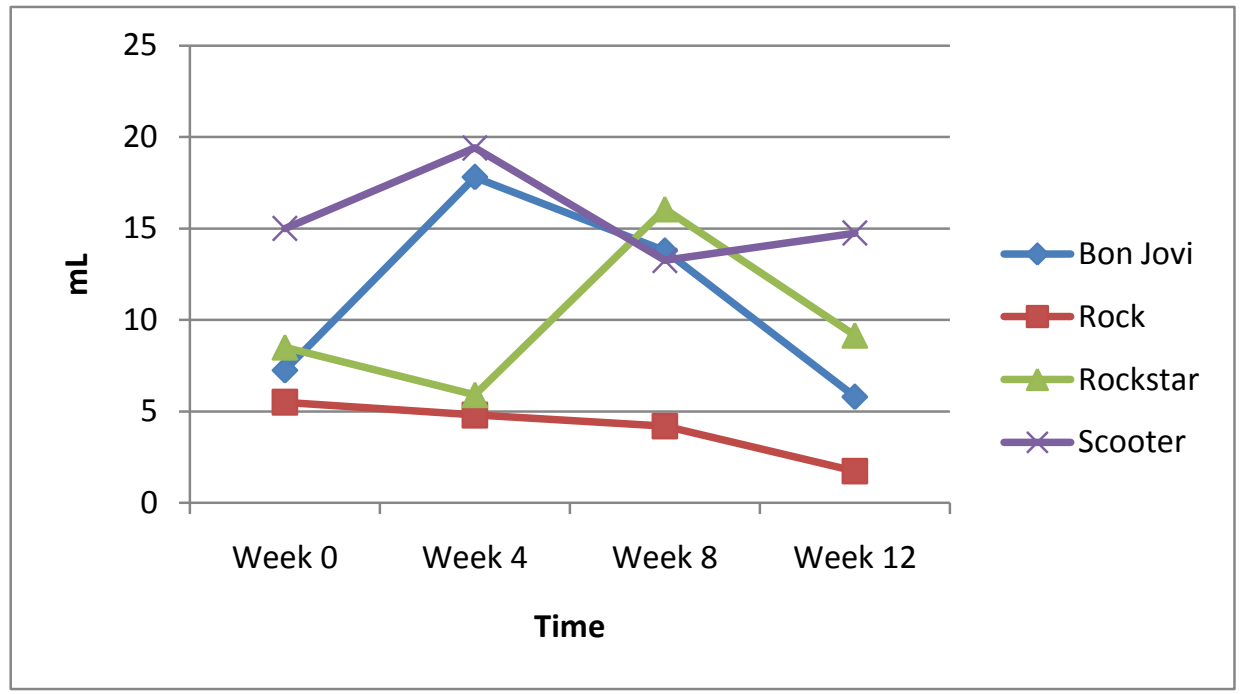

b)

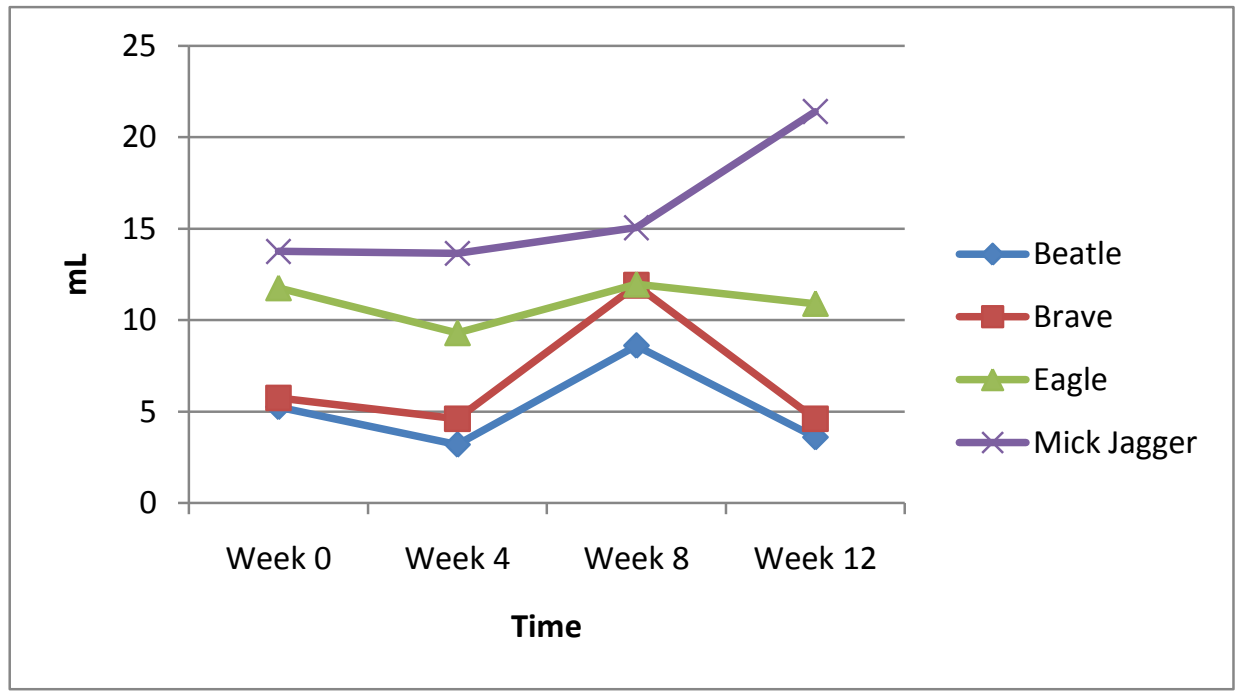

FIGURE E.1 Variations in mean gel-free volume ( $\mathrm{mL})$ of individual stallions within treatment groups: (a) non-exercised (control; $n=4$ ) and (b) exercised (treatment; $n=4$ ). 
a)

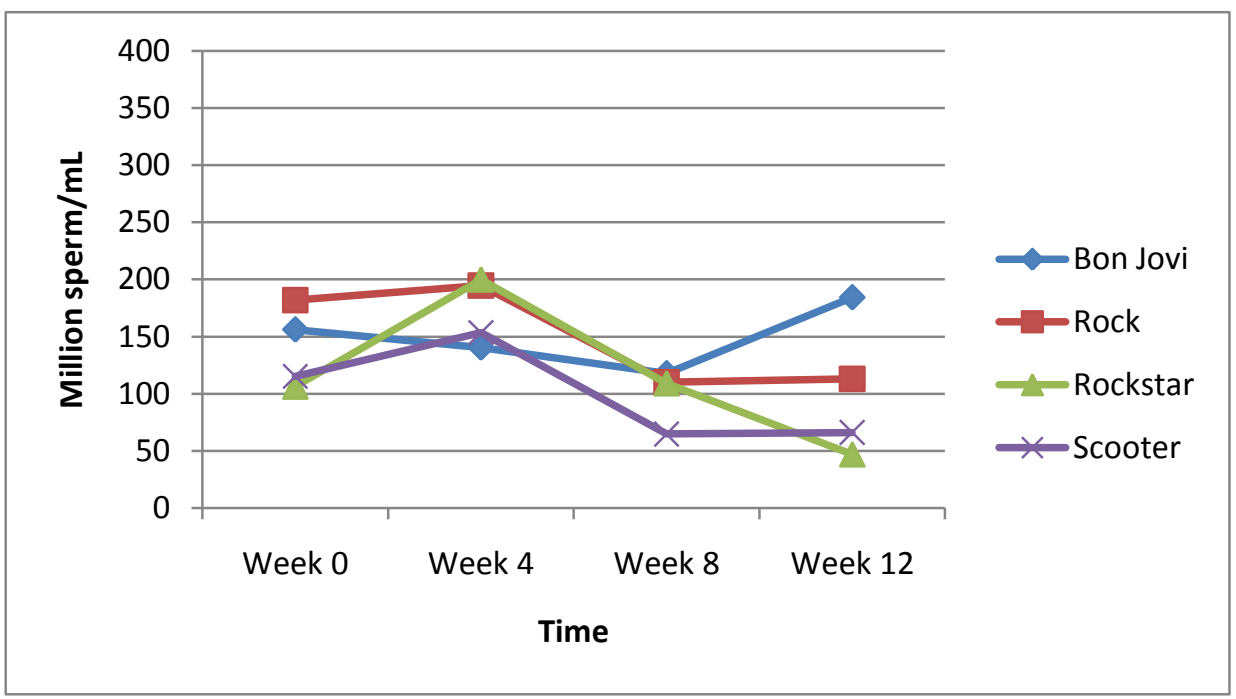

b)

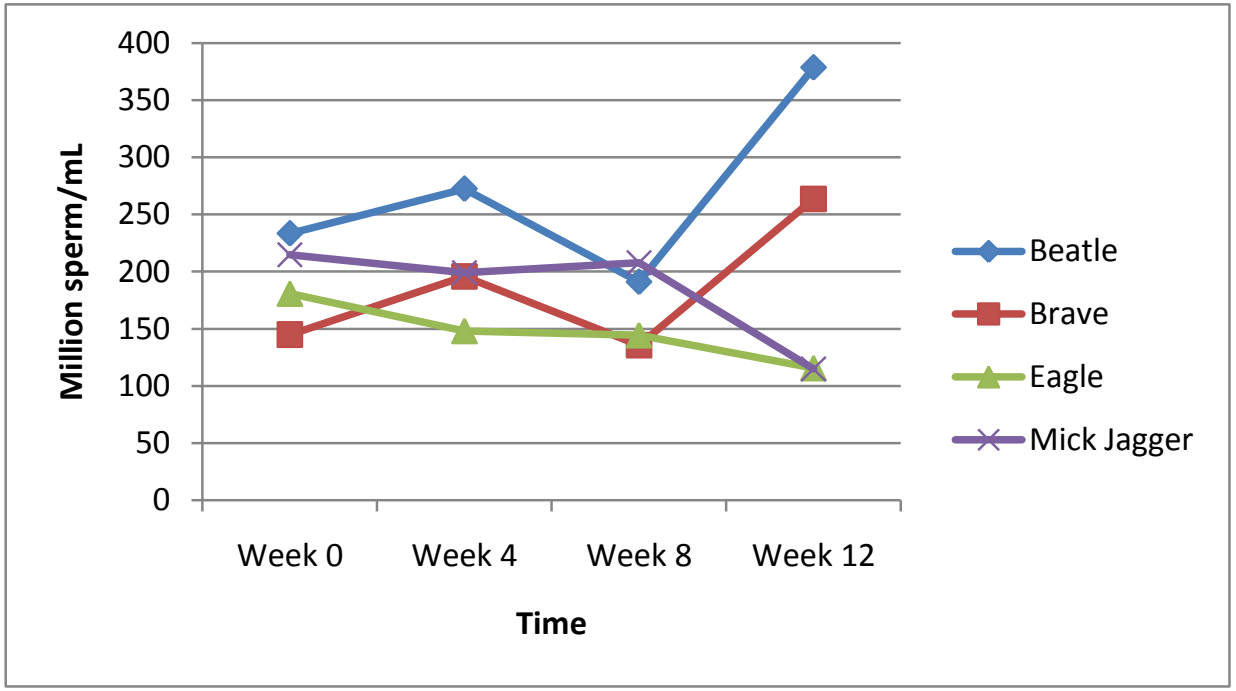

FIGURE E.2 Variations in mean spermatozoal concentration (million spermatozoa/mL) of individual stallions within treatment groups: (a) non-exercised (control; $n=4$ ) and (b) exercised (treatment; $n=4$ ). 
a)

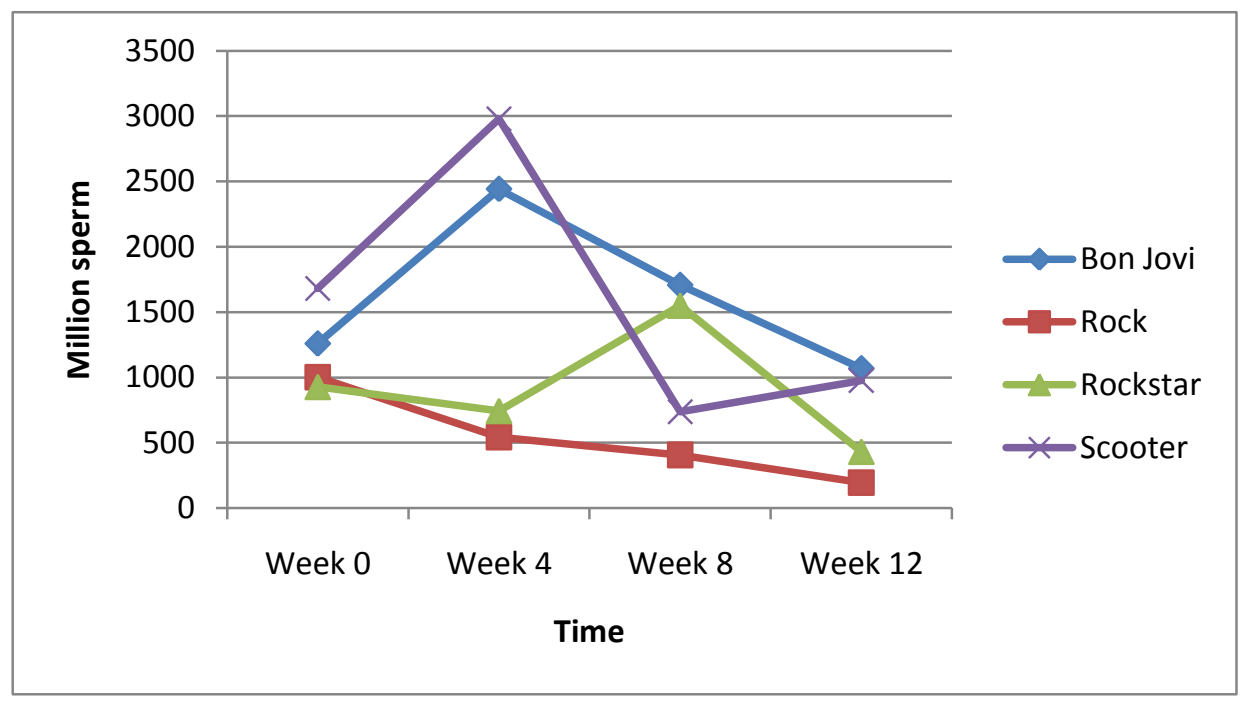

b)

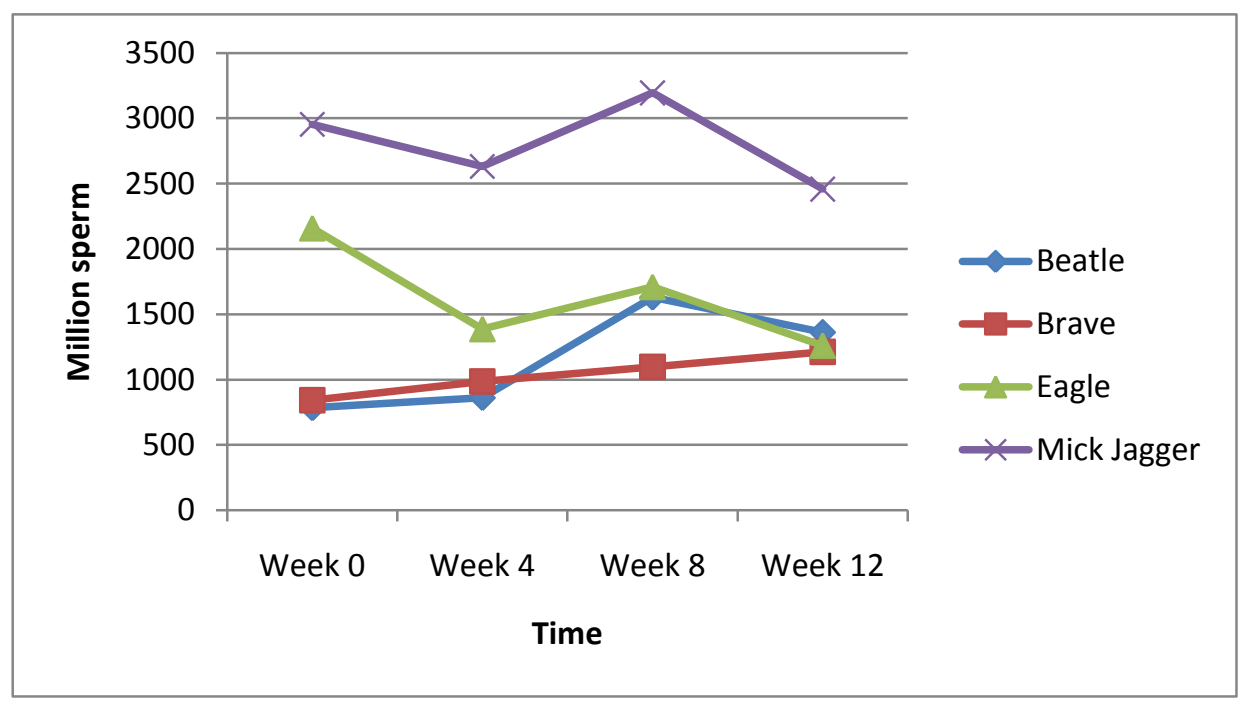

FIGURE E.3 Variations in mean total numbers (million spermatozoa) of individual stallions within treatment groups: (a) non-exercised (control; $n=4$ ) and (b) exercised (treatment; $n=4$ ). 
a)

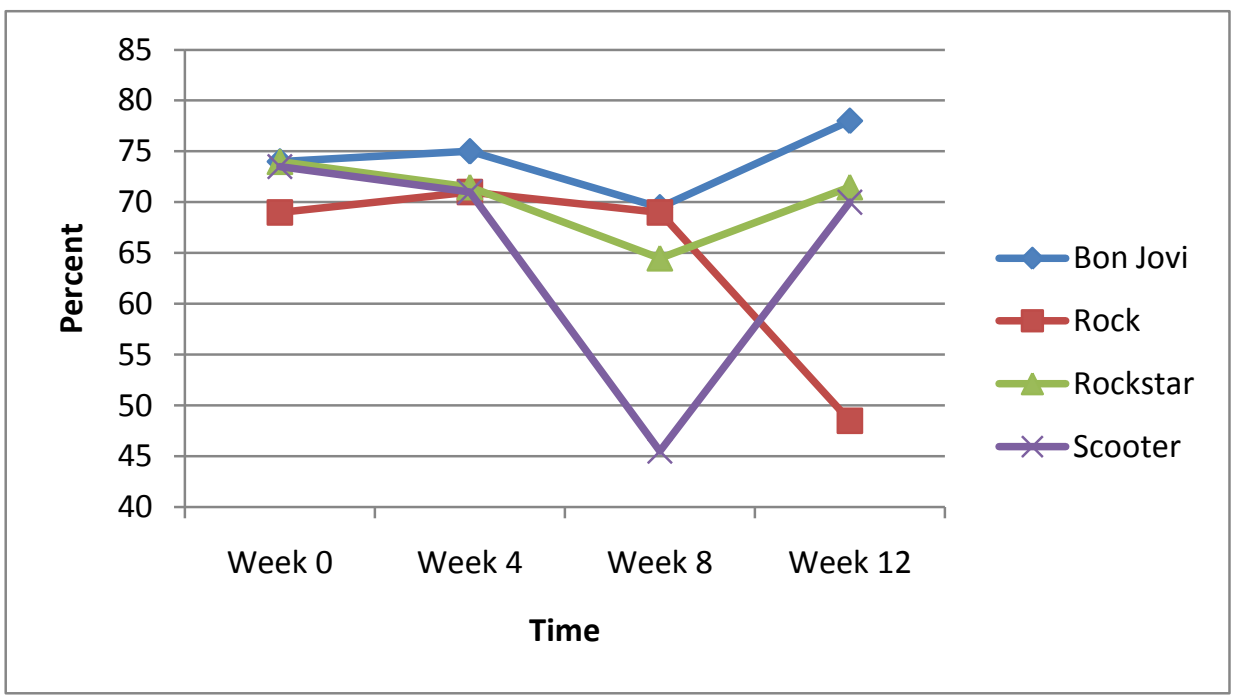

b)

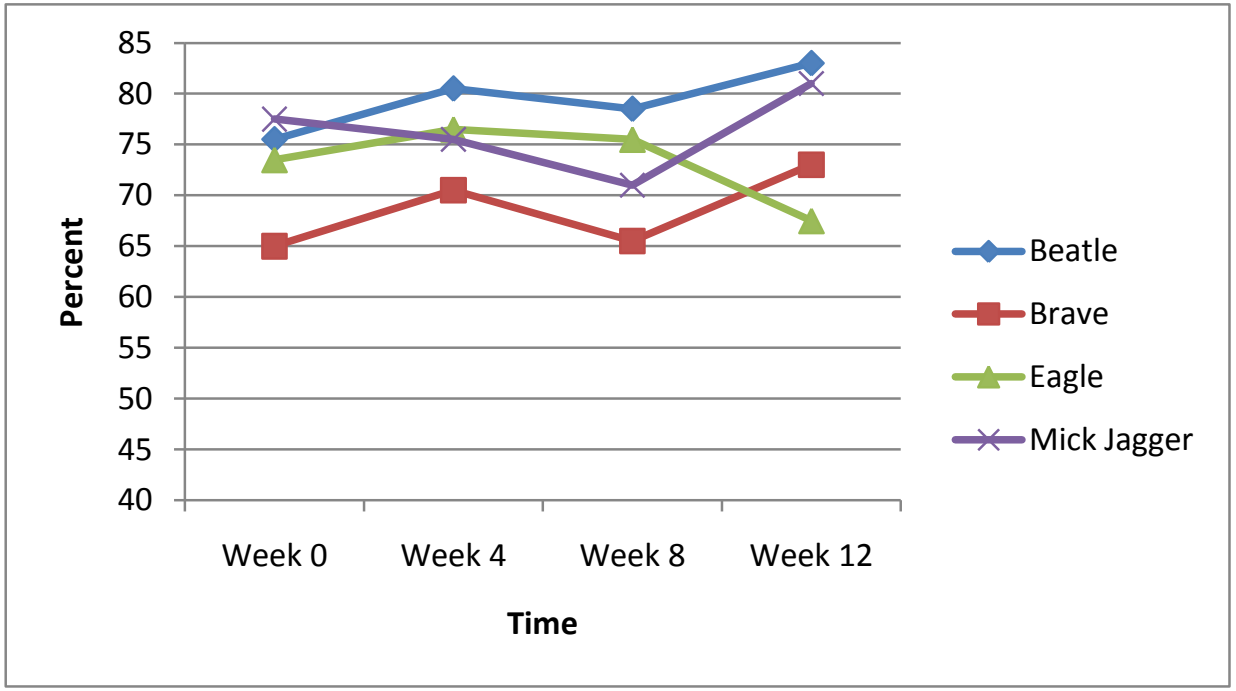

FIGURE E.4 Variations in mean viability (\%) of individual stallions within treatment groups: (a) non-exercised (control; $n=4$ ) and (b) exercised (treatment; $n=4$ ). 
a)

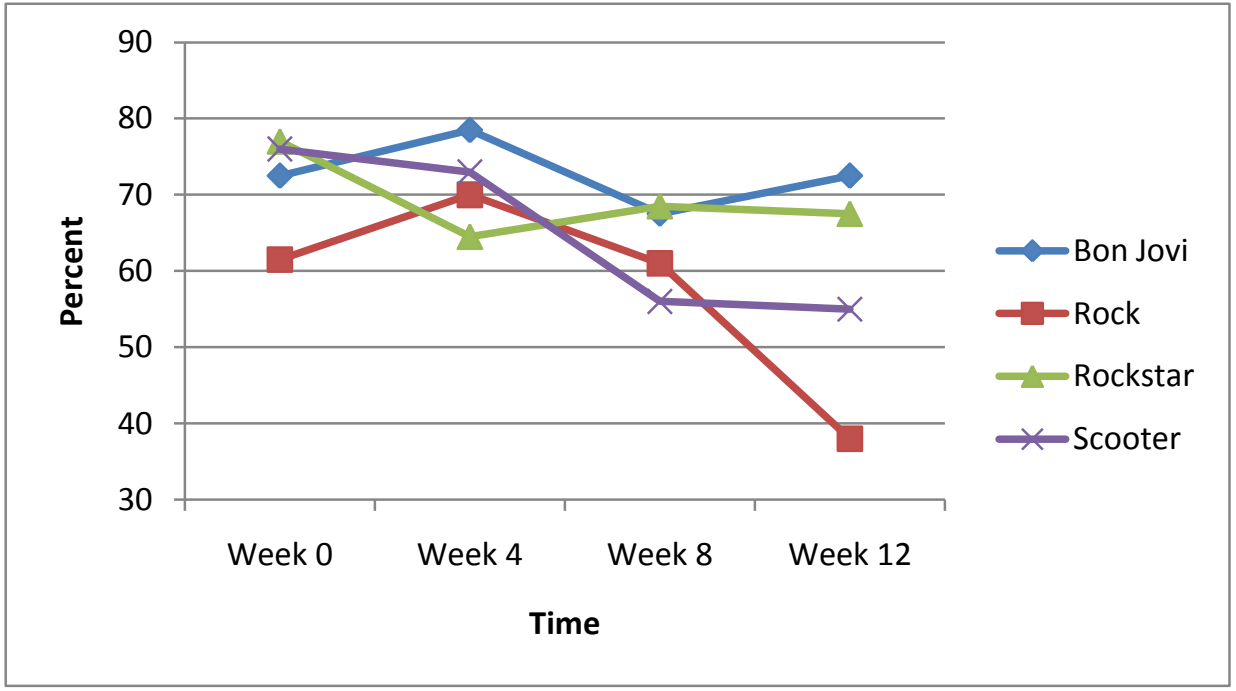

b)

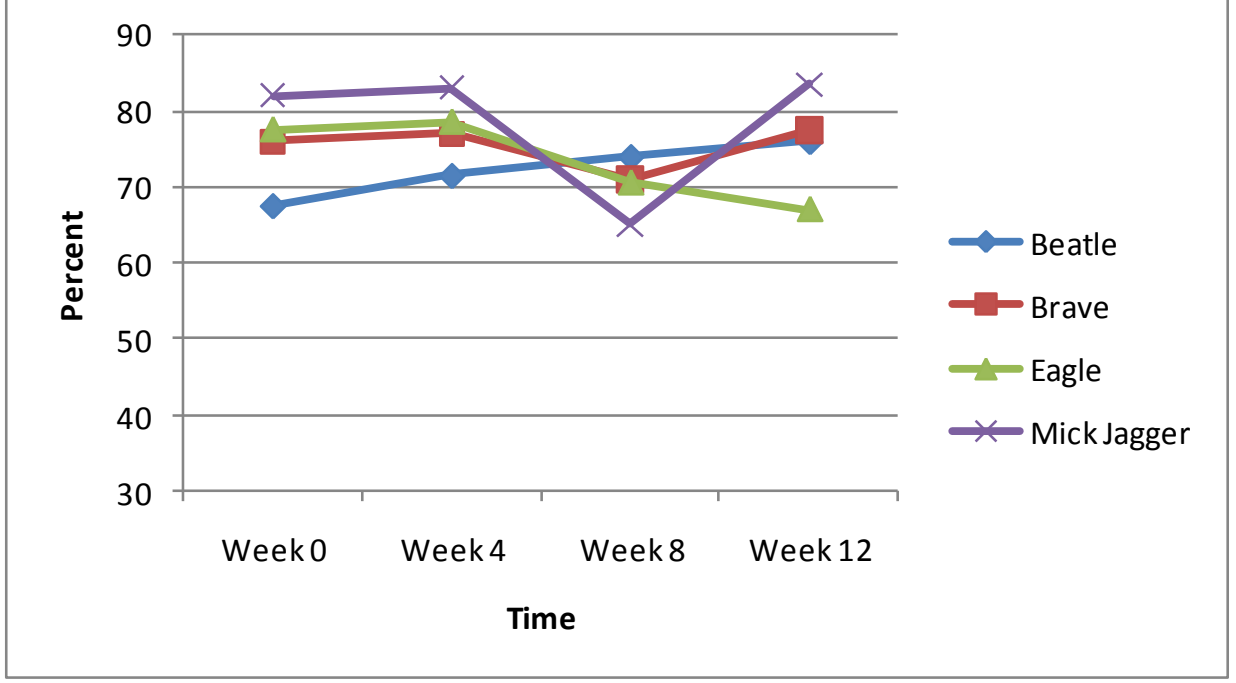

FIGURE E.5 Variations in mean total motility (\%) of individual stallions within treatment groups: (a) non-exercised (control; $n=4$ ) and (b) exercised (treatment; $n=4$ ). 
a)

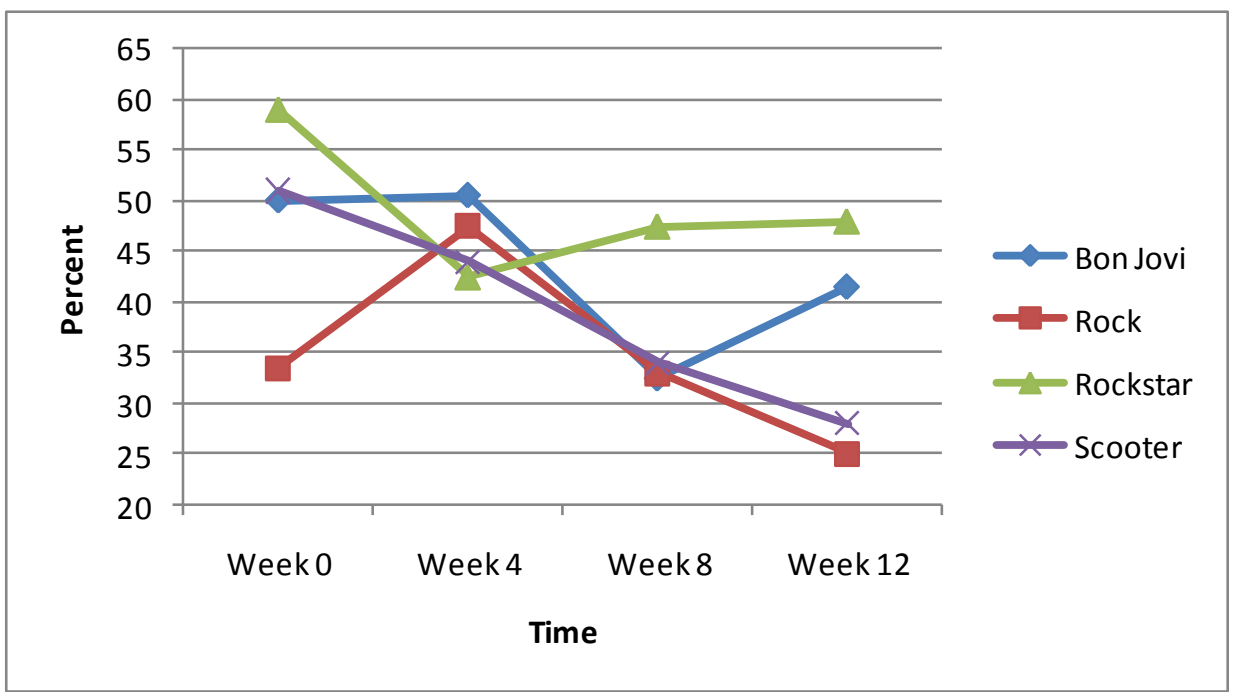

b)

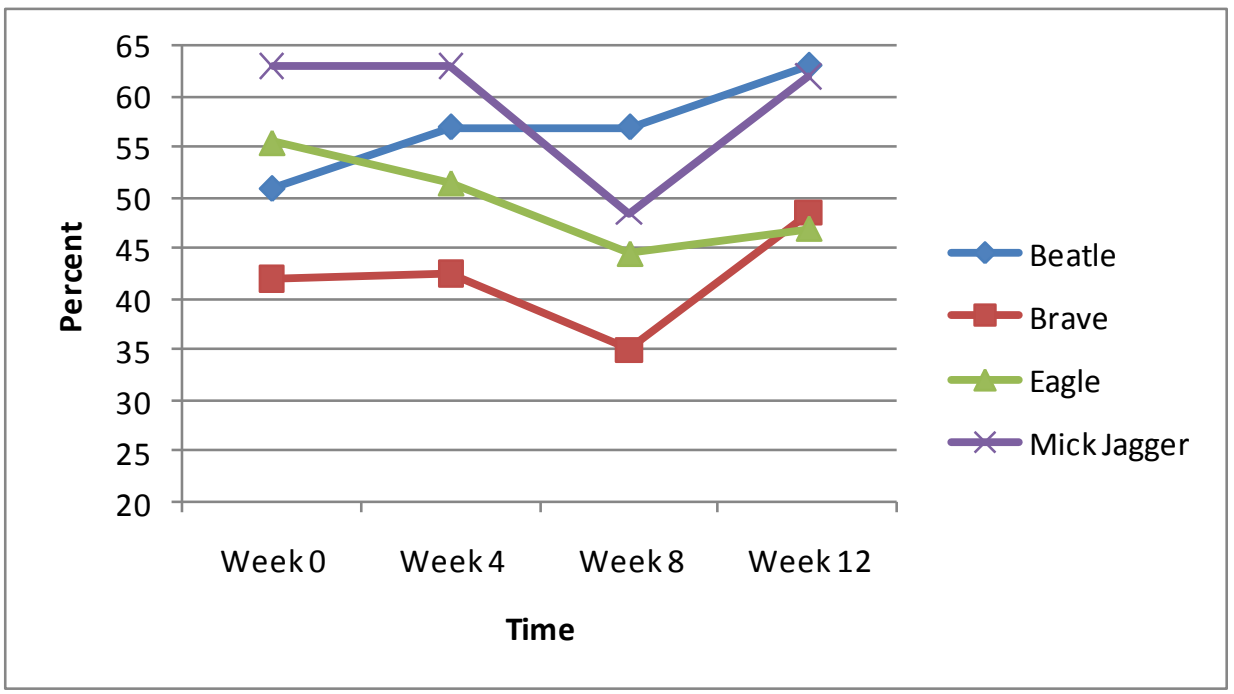

FIGURE E.6 Variations in mean progressive motility (\%) of individual stallions within treatment groups: (a) non-exercised (control; $n=4$ ) and (b) exercised (treatment; $n=4$ ). 
a)

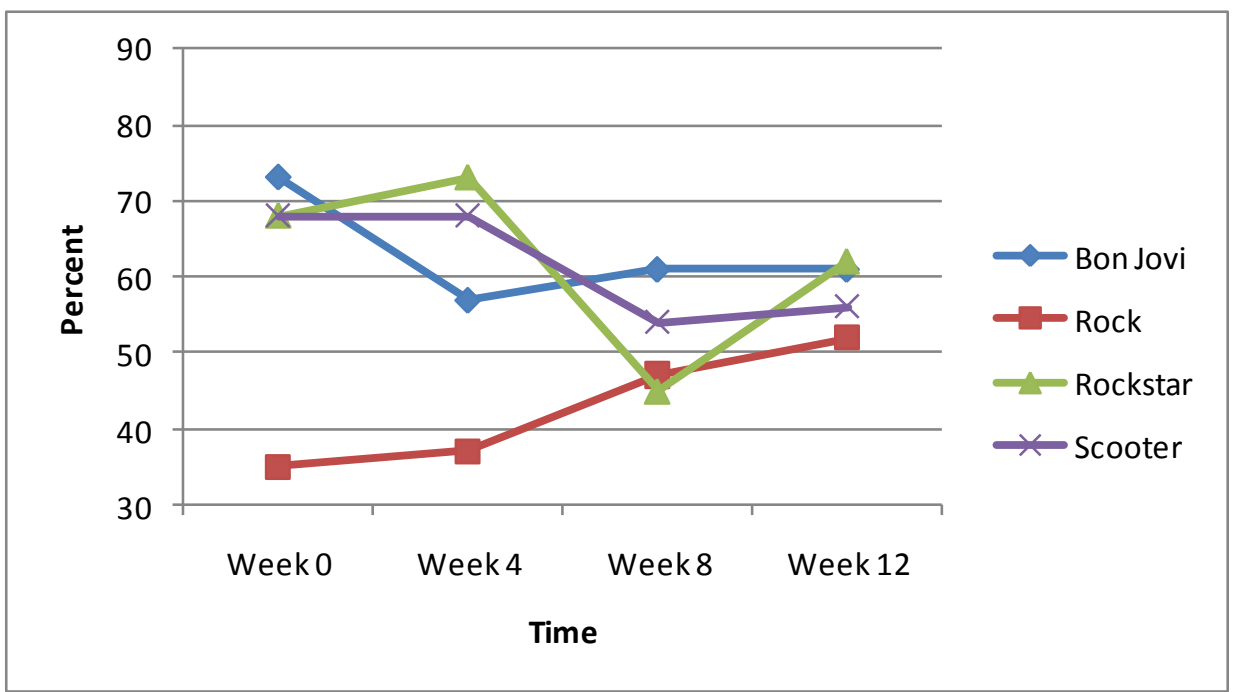

b)

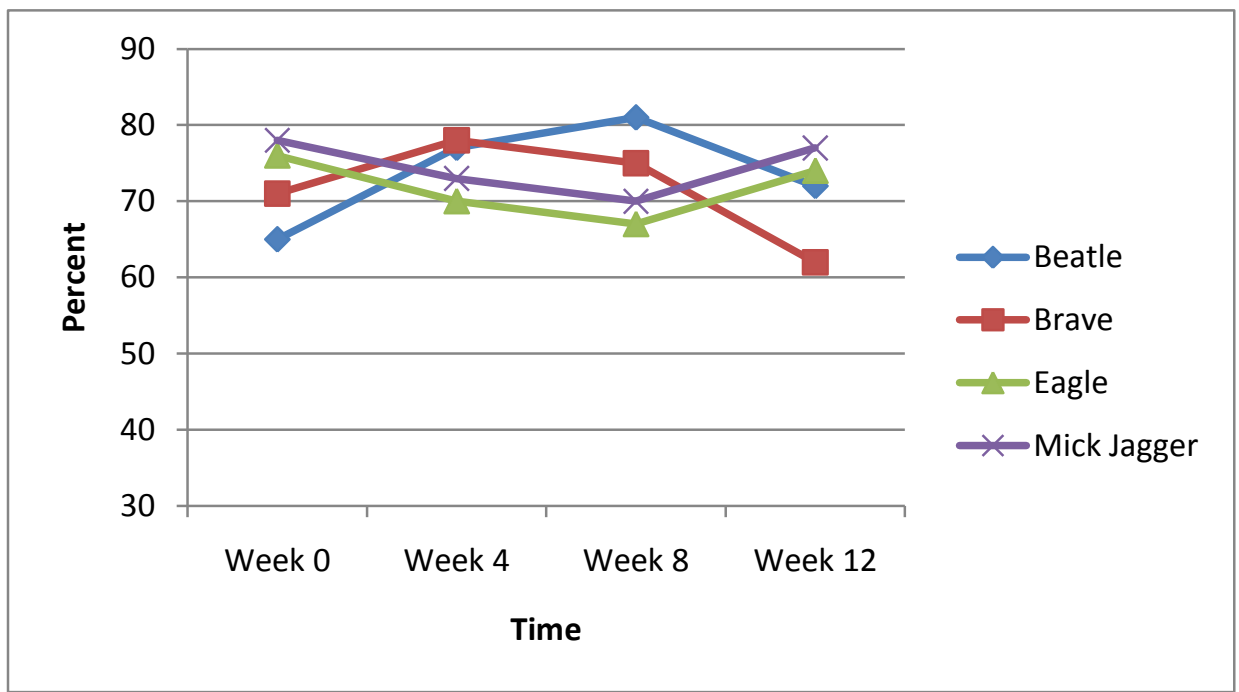

FIGURE E.7 Variations in mean normal spermatozoa (\%) of individual stallions within treatment groups: (a) non-exercised (control; $n=4$ ) and (b) exercised (treatment; $n=4$ ). 


\section{APPENDIX F}

\section{MORPHOLOGICALLY ABNORMAL SPERMATOZOA BETWEEN GROUPS}

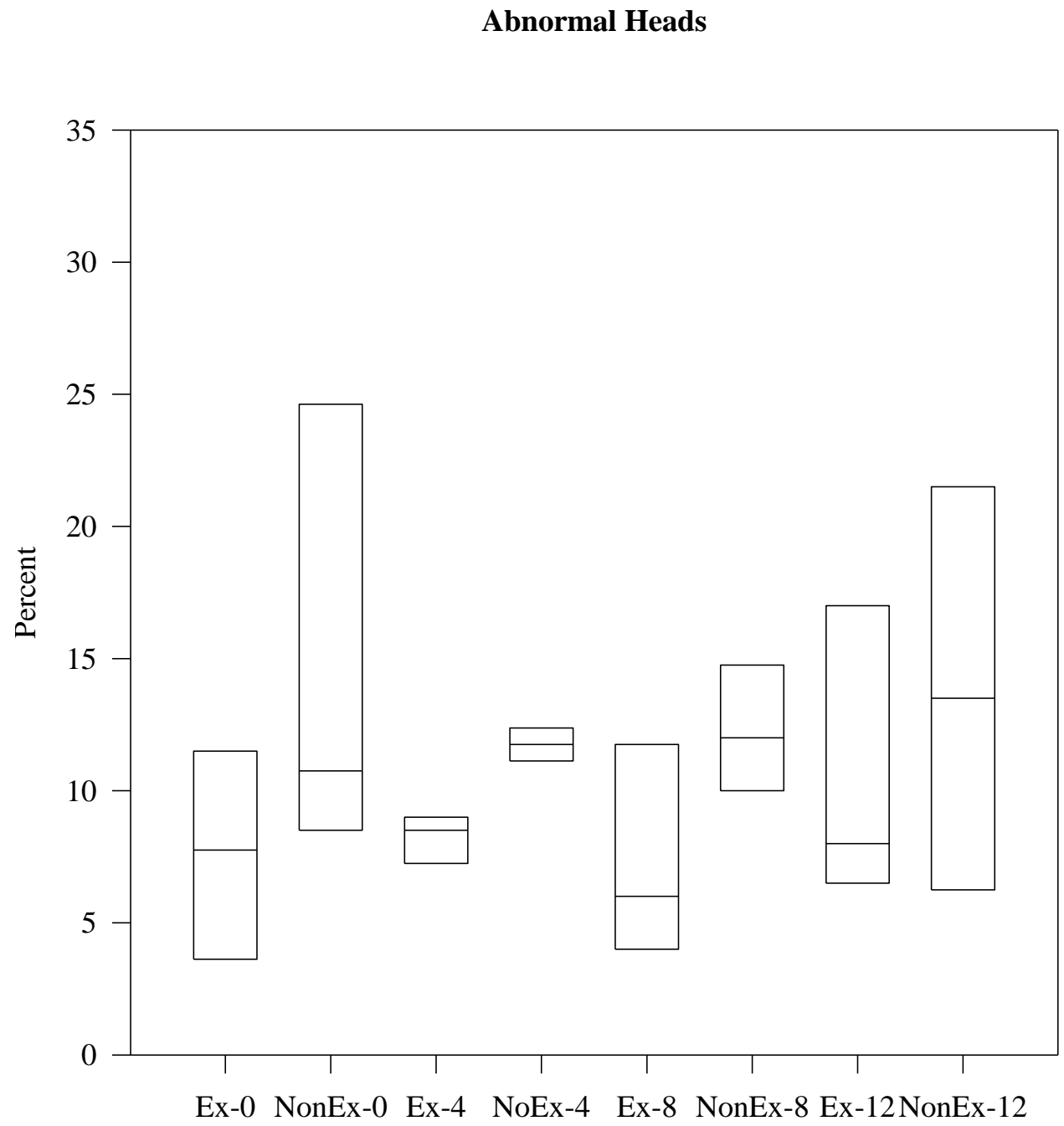

FIGURE F.1 Box plots representing mean percentages of abnormal heads among nonex (control) and ex (treatment) groups at weeks $0,4,8$, and 12 ( $\mathrm{n}=4$ /group). 


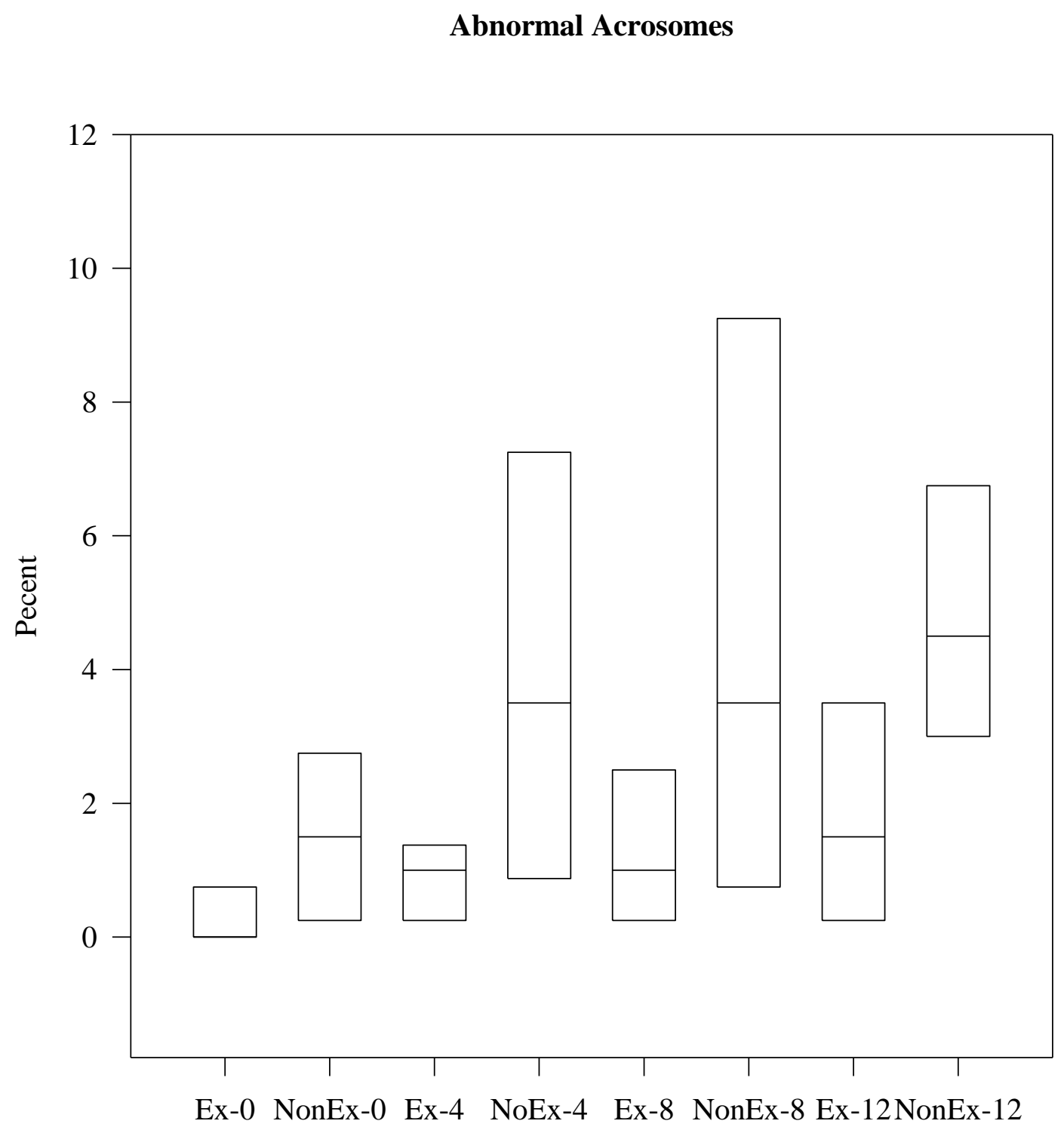

FIGURE F.2 Box plots representing mean percentages of abnormal acrosomes among non-ex (control) and ex (treatment) groups at weeks $0,4,8$, and 12 (n=4/group). 
Detached Heads

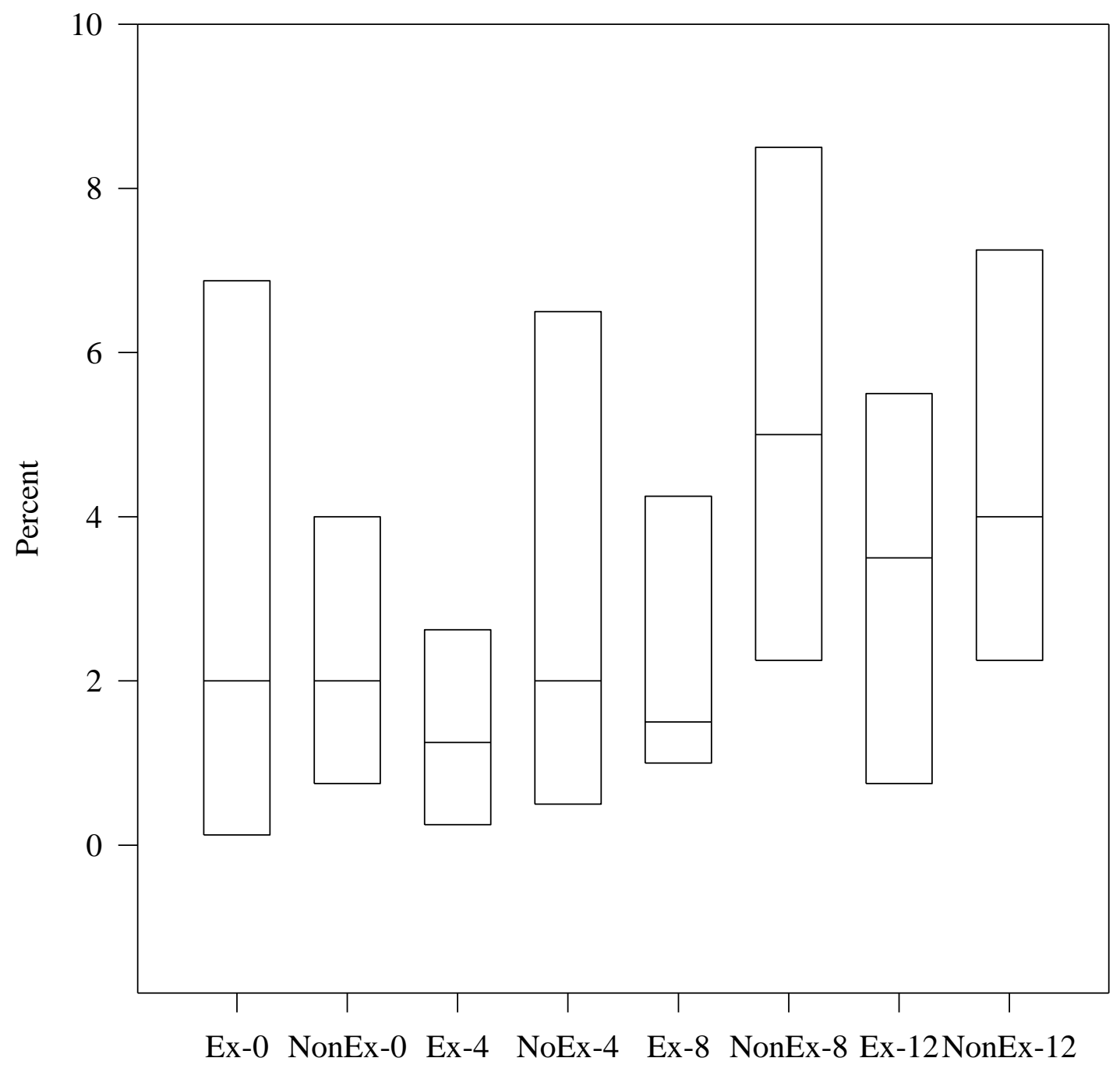

FIGURE F.3 Box plots representing mean percentages of detached heads among nonex (control) and ex (treatment) groups at weeks $0,4,8$, and 12 ( $\mathrm{n}=4$ /group). 


\section{Proximal Droplets}

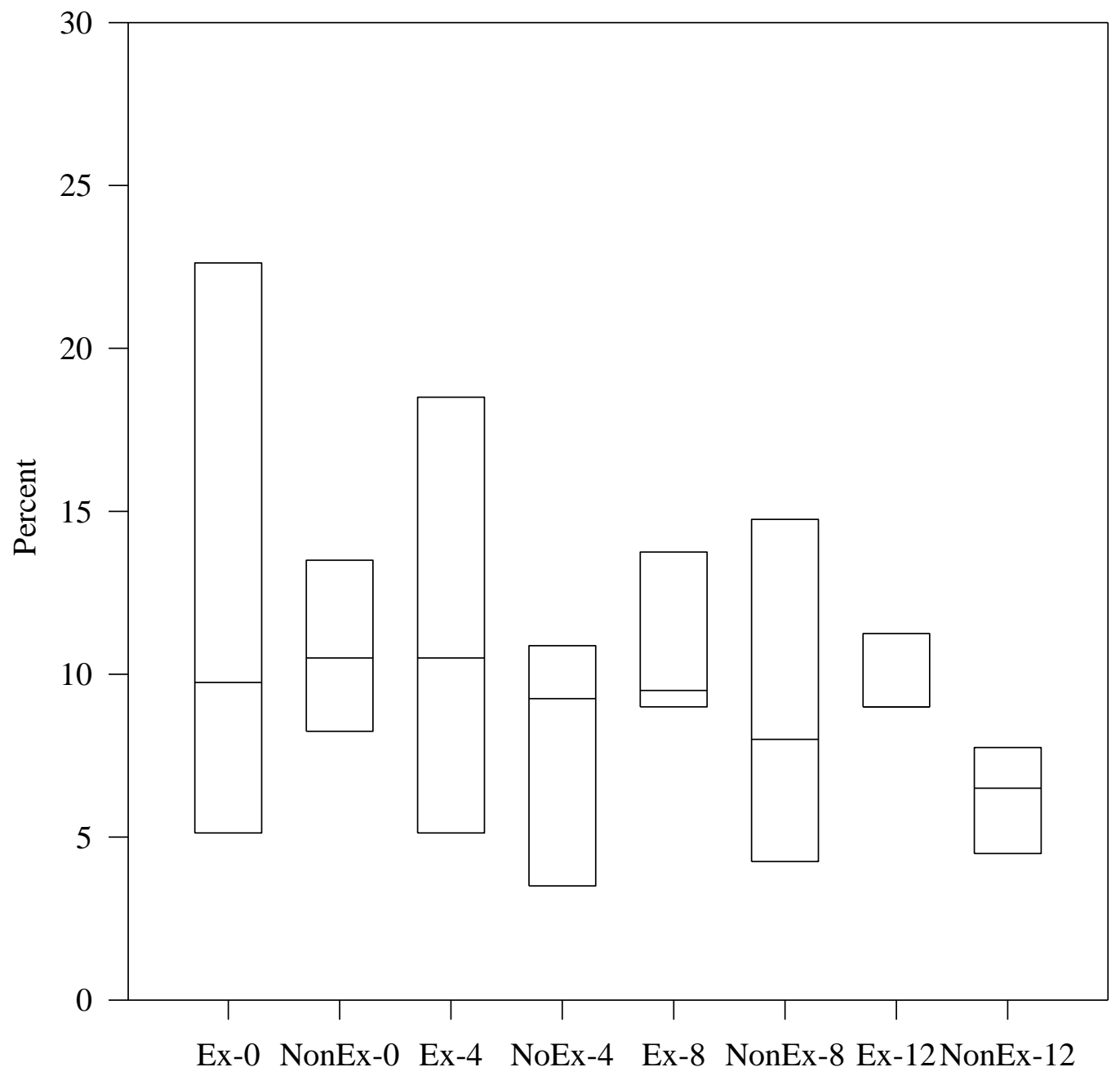

FIGURE F.4 Box plots representing mean percentages of proximal droplets among non-ex (control) and ex (treatment) groups at weeks $0,4,8$, and 12 (n=4/group). 


\section{Distal Droplets}

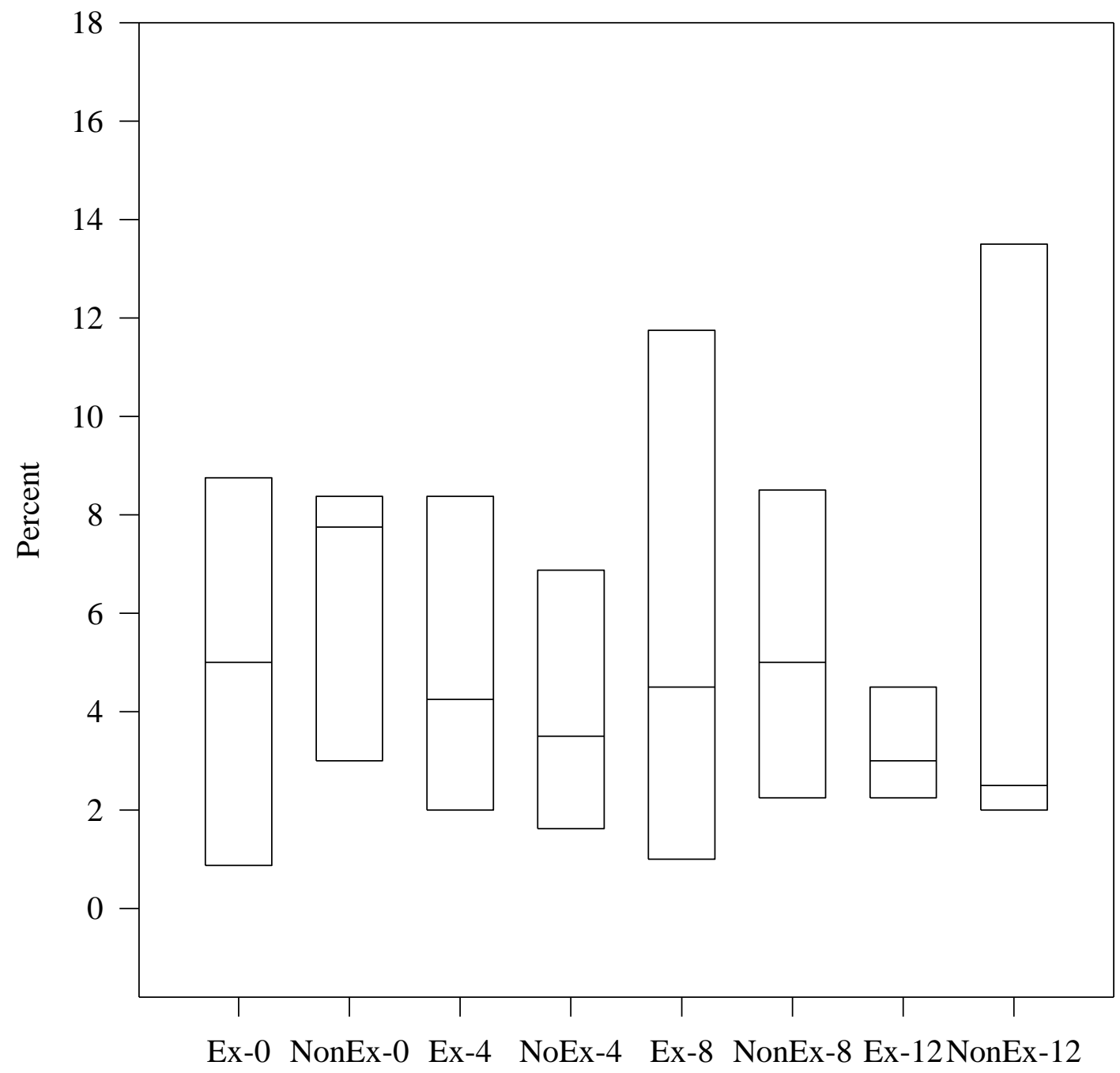

FIGURE F.5 Box plots representing mean percentages of distal droplets among non-ex (control) and ex (treatment) groups at weeks 0, 4, 8, and 12 (n=4/group). 
Abnormal Midpieces

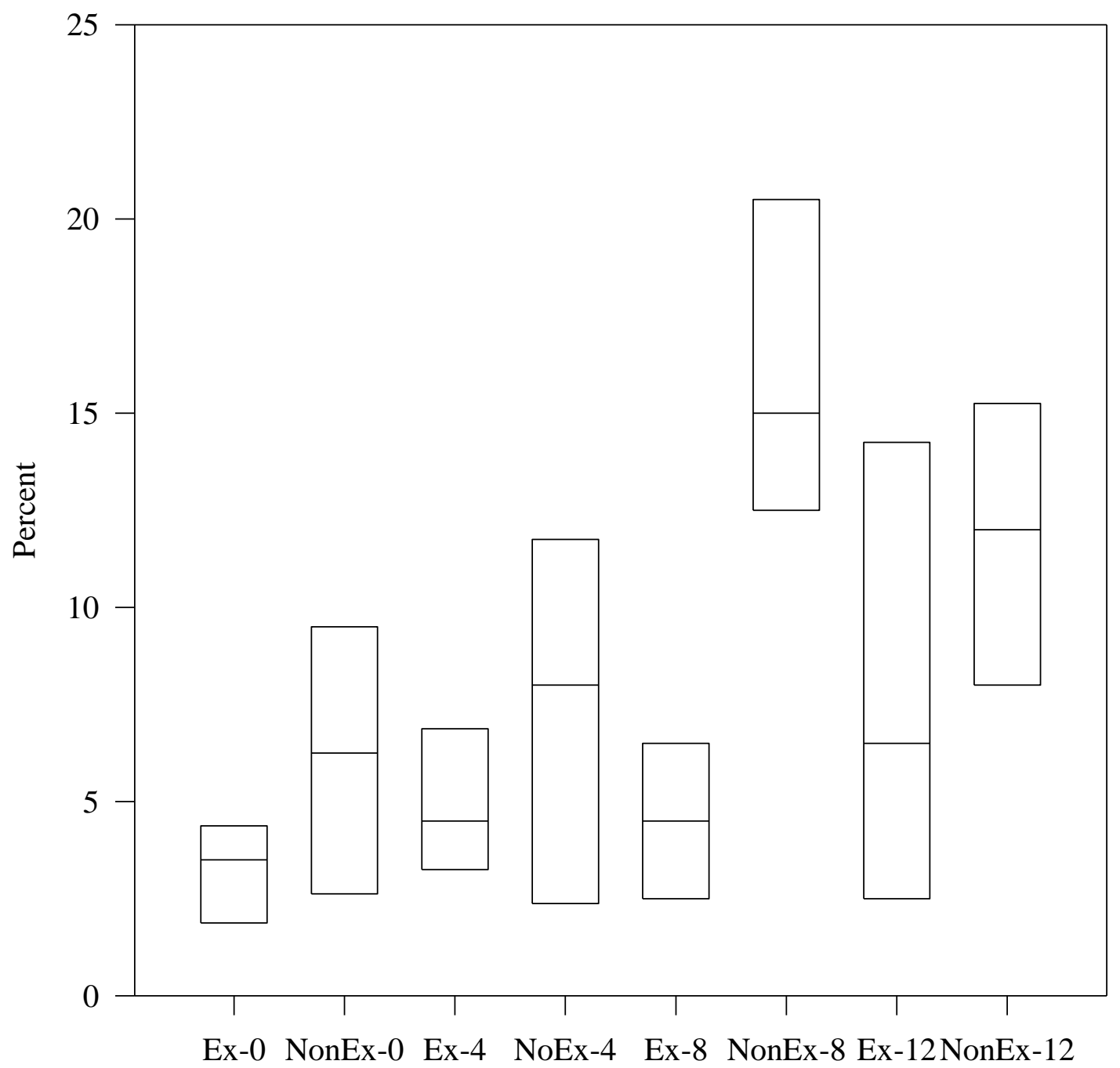

FIGURE F.6 Box plots representing mean percentages of abnormal midpieces among non-ex (control) and ex (treatment) groups at weeks $0,4,8$, and 12 ( $\mathrm{n}=4$ /group). 


\section{Bent Midpieces}

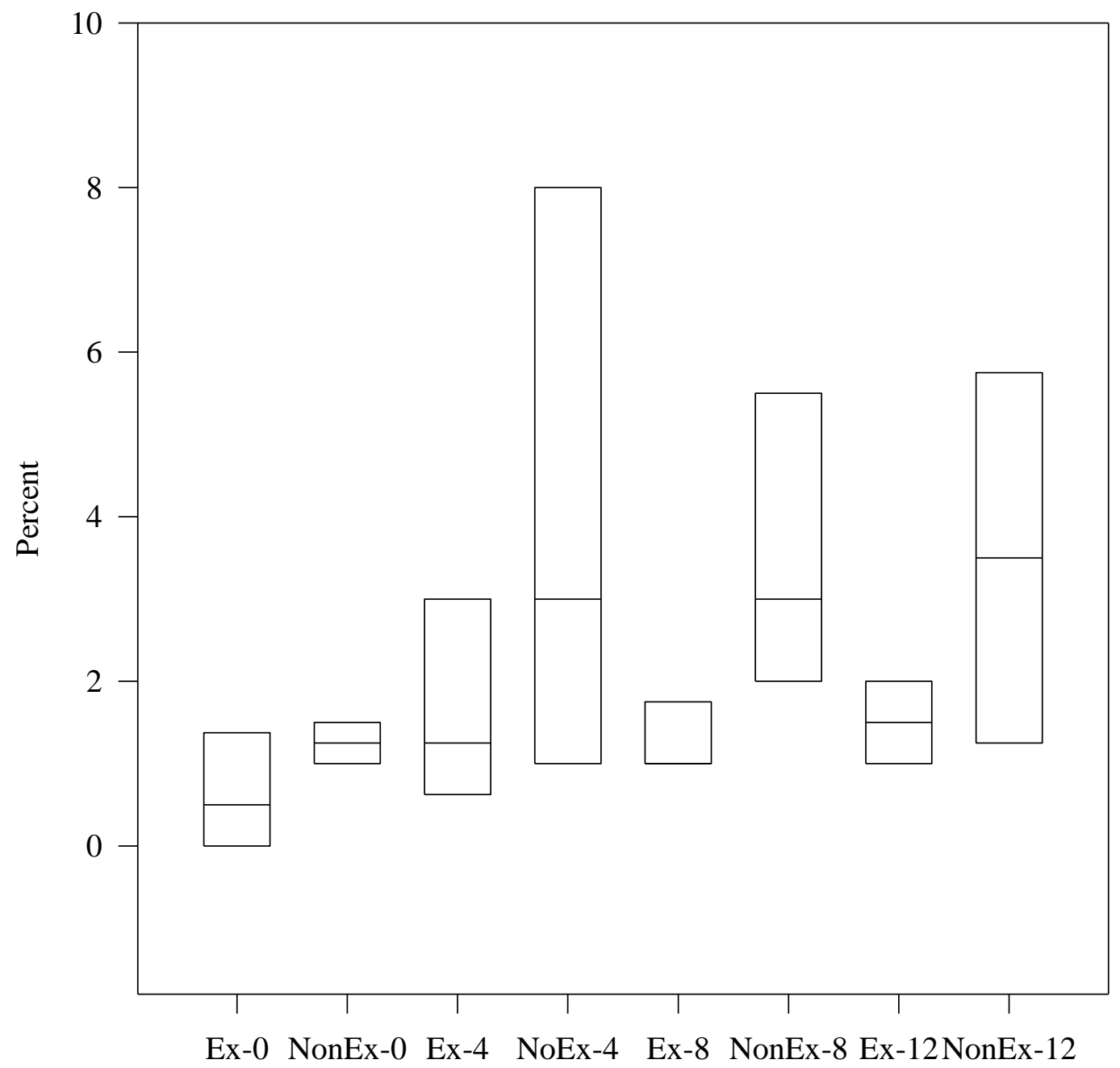

FIGURE F.7 Box plots representing mean percentages of bent midpieces among nonex (control) and ex (treatment) groups at weeks $0,4,8$, and 12 (n=4/group). 


\section{Bent Tails}

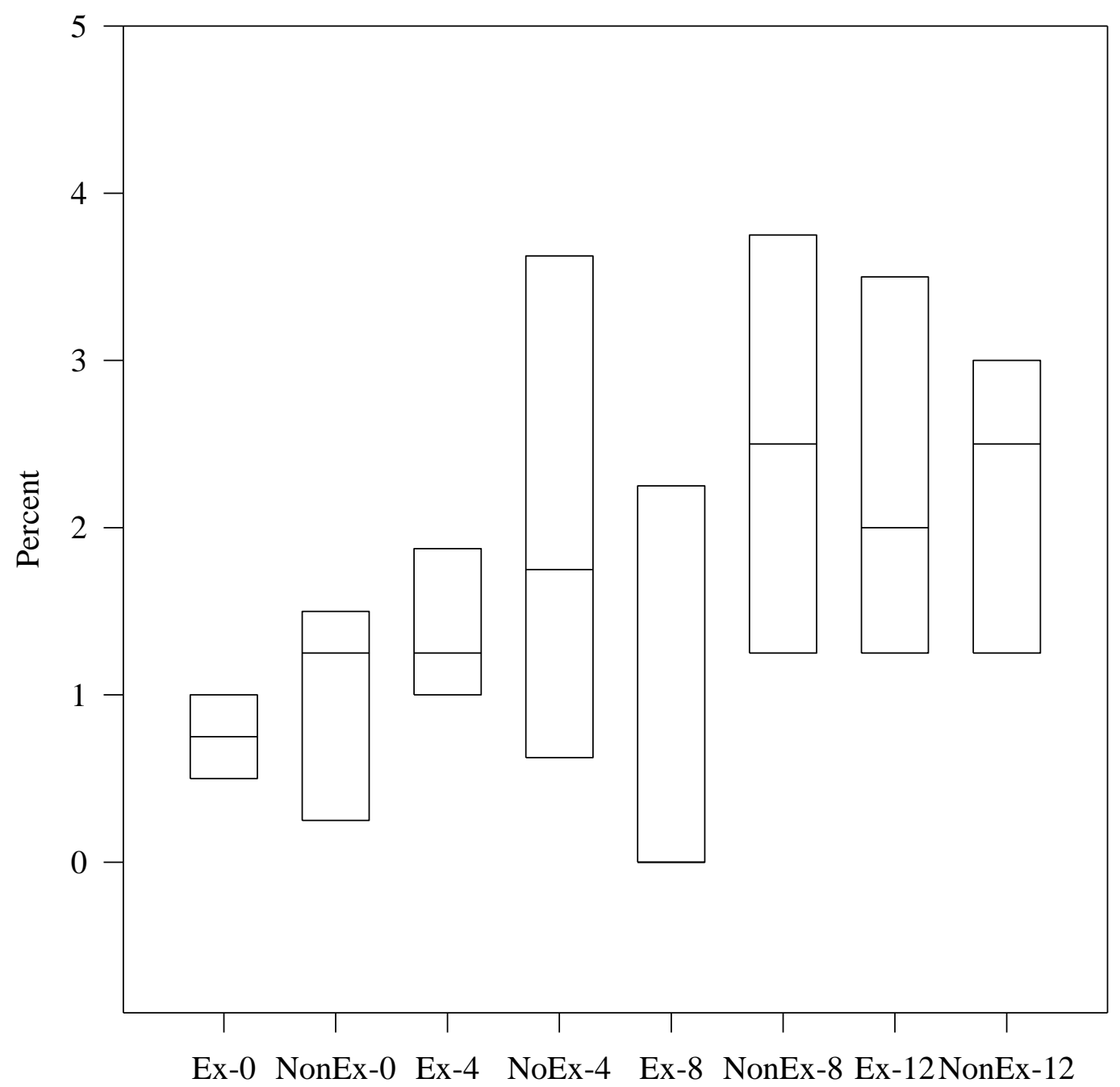

FIGURE F.8 Box plots representing mean percentages of bent tails among non-ex (control) and ex (treatment) groups at weeks $0,4,8$, and 12 ( $\mathrm{n}=4$ /group). 
Coiled Tails

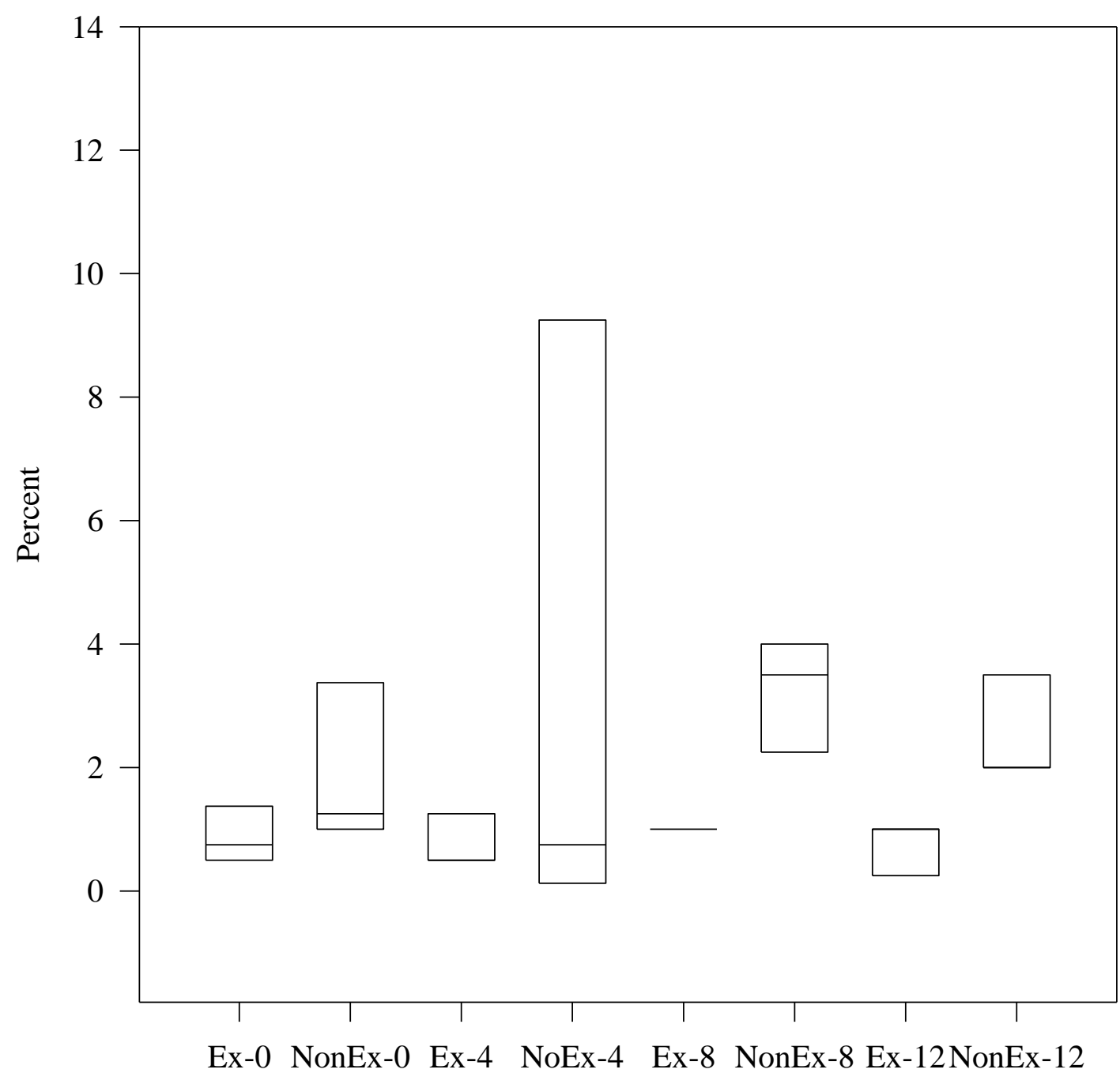

FIGURE F.9 Box plots representing mean percentages of coiled tails among non-ex (control) and ex (treatment) groups at weeks 0, 4, 8, and 12 (n=4/group). 


\section{Premature Germ Cells}

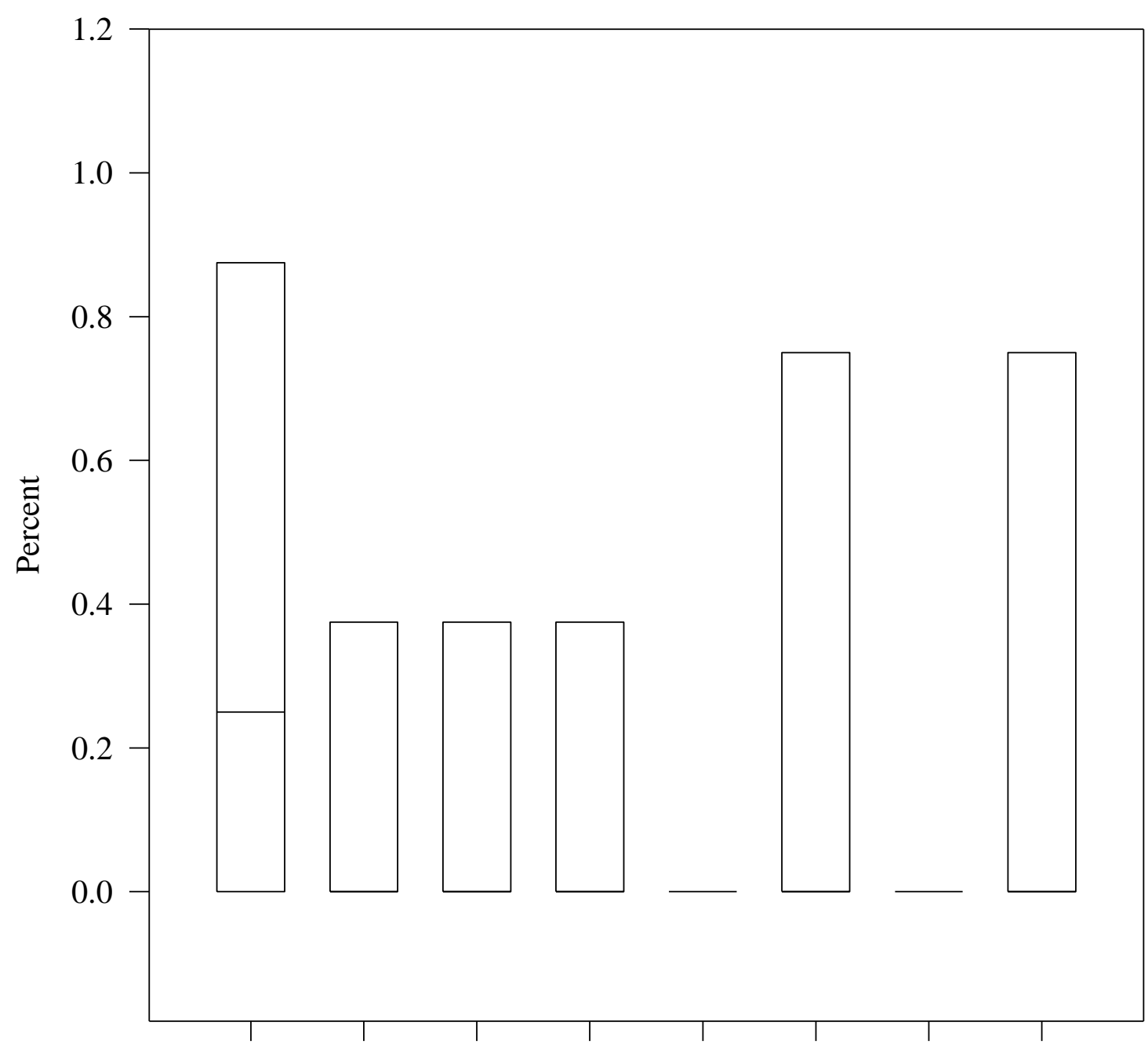

Ex-0 NonEx-0 Ex-4 NoEx-4 Ex-8 NonEx-8 Ex-12NonEx-12

FIGURE F.10 Box plots representing mean percentages of premature germ cells among non-ex (control) and ex (treatment) groups at weeks $0,4,8$, and 12 (n=4/group). 


\section{APPENDIX G}

STANDARD EXERCISE TEST FIGURES

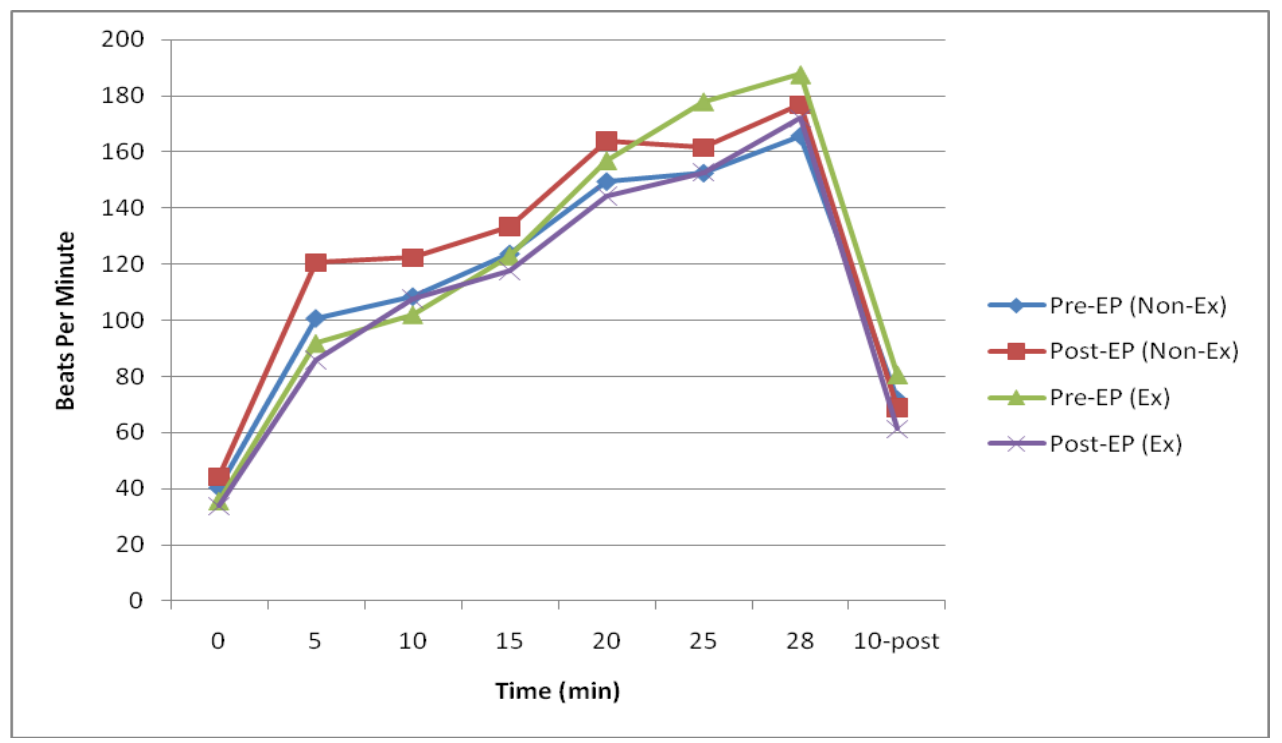

FIGURE G.1 Mean heart rates (beats per minute) of non-ex (control; $n=4)$ and ex (treatment; $n=4$ ) stallions during standard exercise tests prior to (pre-EP) and following (post-EP) the exercise period.

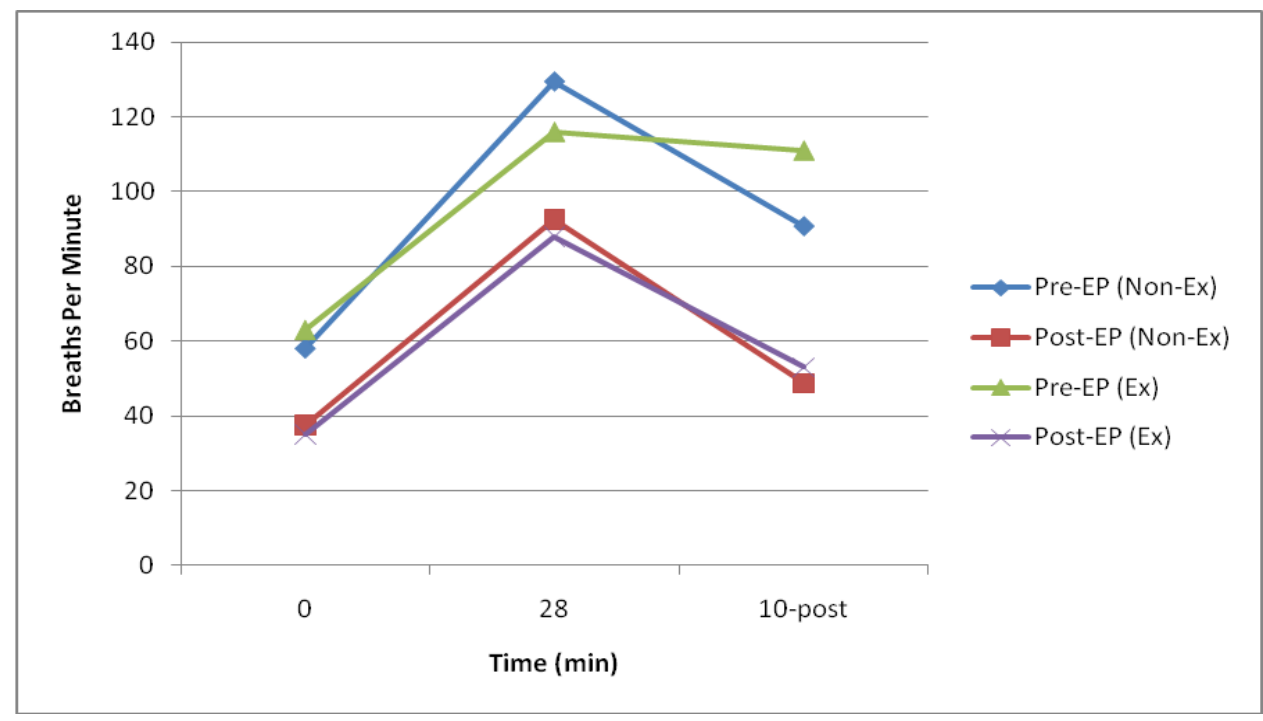

FIGURE G.2 Mean respiration rates (breaths per minute) of non-ex (control; $n=4$ ) and ex (treatment; $n=4)$ stallions during standard exercise tests prior to (pre-EP) and following (post-EP) the exercise period. 


\section{VITA}

Name:

Jeannette Diane Mawyer

Address:

2471 Kleberg Bldg.

Texas A\&M University

College Station, TX 77843

Email Address: $\quad$ jeannette.mawyer@tamu.edu

Education: $\quad$ B.S.A., Animal Health Technology

Murray State University, 2008 